NISTIR 7571

\title{
Estimating Volumes of Simulated Lung Cancer Nodules*
}

\author{
David E. Gilsinn ${ }^{\dagger}$ \\ Bruce R. Borchardt ${ }^{\ddagger}$ \\ Amelia Tebbe ${ }^{\S}$
}

February 23, 2009

\begin{abstract}
Lung cancer is a disease of uncontrolled cell growth in tissues of the lung. Computed tomography (CT) shows promise in detecting lung cancers at earlier, more operable stages, when survival is better. CT scans generate multiple 2-D slice images of the lung and digital image processing software is used to combine these images into a 3-D representation of the lung and, in particular, an identified cancer lesion. Various CT scanners use, often different and usually proprietary, software to develop these 3-D images and generate parameters such as lesion volume. Tracking lesion volume is considered a good diagnostic tool for evaluating the results of patient treatment. The Food and Drug Administration (FDA) is conducting research on developing reference cancer lesions, called phantoms, to test CT scanners and their proprietary software. FDA loaned two semi-spherical phantoms to NIST, called Green and Pink, and asked to have the phantoms measured by a coordinate measuring machine (CMM) and the volumes estimated. This report describes in detail both the experimental and computational methods used to estimate the phantoms' volumes as well as a bootstrap method for estimating the uncertainties of the computed volumes. Three sets of CMM measured data were produced. One set of data involved data density measurements of a known calibrated metal sphere. The other two sets were measurements of the two FDA phantoms at two densities, called the coarse set and the dense set. Two computational approaches were applied to the data. In the first approach spherical models were fit to the calibrated sphere data and to the phantom data. The second approach was to model the data points on the boundaries of the spheres with surface B-splines and then use the Divergence Theorem to estimate the volumes. The results for the coarse data set tended to predict the volumes as expected with low expanded uncertainties and the results did show that the Green phantom was very near spherical. This was confirmed by both computational methods. The spherical model did not fit the Pink phantom as well and the B-spline approach provided a better estimate of the volume in that case. The results for the dense data set did not provide as nice a prediction of volumes as the coarse data set and produced larger expanded uncertainties. A discussion of some possible reasons for these results will be given in the conclusion section. The report includes the MATLAB codes used in the study.
\end{abstract}

Keywords: B-splines, computed tomography, coordinate measuring machine, divergence theorem, lung cancer, lung cancer phantoms, nonlinear least squares.

\footnotetext{
*Contribution of the National Institute of Standards and Technology, a Federal agency, not subject to copyright.

$\dagger$ Mathematical and Computational Sciences Division, Information Technology Laboratory, National Institute of Standards and Technology, Gaithersburg MD 20899-8910.

‡Precision Engineering Division, Manufacturing Engineering Laboratory, National Institute of Standards and Technology, Gaithersburg MD 20899-8211.

$\S$ Department of Mathematics, St. Mary's College of Maryland, St. Mary's City, MD 20686-3001.
} 


\section{Introduction}

Lung cancer is a disease of uncontrolled cell growth in tissues of the lung. It is the most common cause of cancer related deaths in men and second most in women and is responsible for an estimated 560,000 deaths in the US in 2007. It is classified as small cell (13\%) and non-small cell (87\%) [12]. The most common cause is long term exposure to tobacco smoke. Lung cancer lesions may be seen in chest X-ray and computed tomography $(\mathrm{CT})$, although chest X-ray analysis has shown limited effectiveness in improving survival. Computed tomography has shown promising results in detecting lung cancers at earlier, more operable stages, when survival is better [1].

Tomography is a method of imaging a single slice of the body, in this case the lung. Modern $\mathrm{CT}$ is a medical imaging method that uses tomography but also employs digital image processing techniques to generate three dimensional images built from a large sequence of two-dimensional Xray images made around a single axis. There are a number of current CT methods but the one that is of most interest in this study is called a helical or spiral CT in which the X-ray source is connected to a rotating gantry. The patient, on a table, moves smoothly through the scanner. The name, helical or spiral CT, comes from the helical path traced out by the X-ray beam. The helical trajectory of the data is converted into two-dimensional image slices of the patient's body. The advantage of the helical or spiral CT is speed, since the patients must remain perfectly still and hold their breath. A large portion of the body can be scanned in 20-60 seconds [11]. CT provides excellent diagnostics for detecting changes in the lungs, where two-dimensional X-rays do not clearly show the lung cancer changes. A diagnosis is usually confirmed by biopsy. Treatments include surgery and chemotherapy. With treatment, the one-year survival was $42 \%$ in 2002 but the five-year survival rate was only $16 \%$ [1].

Evaluating the rate of growth/shrinkage of lung cancer lesions is important for pharmaceutical research in order to compare drug effects, for clinical practice to determine time to use drugs or surgery, and for patient follow up to quantify the effects of drugs or surgery. A goal then is to enable CT scans to become a measurement device, providing quantitative rather than only qualitative information. The current guidelines for measuring the increase or decrease of tumors involve linear measurements of the greatest diameter of a lesion from the sequence of CT slices [19]. However, the volume change of lesions is found to show more significant relative change than a relative diameter change. As an example, for a sphere a $30 \%$ decrease in the relative diameter would produce a $65.7 \%$ decrease in relative volume whereas a $30 \%$ increase in the relative diameter would produce approximately a $120 \%$ increase in relative volume.

The Food and Drug Administration (FDA) is conducting research on developing reference cancer lesions, called phantoms, to test CTs and their proprietary software. Two samples were loaned to NIST to estimate volumes. The material of which the phantoms are composed simulates lung cancer material. They can be inserted into a simulated body torso for CT scans. The two phantoms are shown in Fig. 1. Although they seem spherical they are slightly non-spherical. The larger one on the right is referred to as the Green phantom and the one on the left is called the Pink phantom due to the material colors

One approach to estimating the volume of the phantoms is to use what is called an Archimedes test in which the phantoms would be immersed in a liquid bath in a well calibrated container with fine measurement gradations to determine liquid displacement. However, in the case of these phantoms, the material used to manufacture them is porous and the phantoms would have to be coated. This, of course, would affect the "ground truth" volume estimate. Furthermore, the potential expense involved with the process was found to be sufficiently significant that another approach was chosen.

The approach chosen for this study is based on a fundamental theorem in calculus, called the Divergence Theorem (see Taylor [17]), which is an analogue of Green's Theorem in two dimensional 


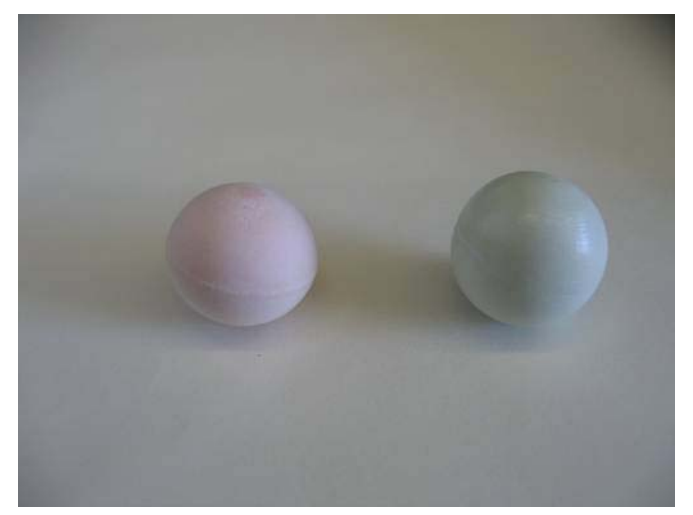

Figure 1: Two simulated lung cancers, called phantoms.

space. In the Divergence Theorem a volume integral is shown to be equal to a surface integral. Therefore, we surmised that, if a model of the surfaces of the phantoms could be developed, we could use the Divergence Theorem to estimate their volumes. In order to develop a surface model we needed to obtain data about the surface. This was done using a coordinate measuring machine (CMM) in the Manufacturing Engineering Lab (MEL) at NIST. This machine produced a set of $(x, y, z)$ points on the surface of each phantom. The data were then transformed to spherical coordinates and modeled using a set of basis functions, called B-splines. After fitting, the B-spline model was then used to generate a grid of values on the surface. These values were used to form surface triangles that were then used to compute the necessary surface integrals and finally the volume. The quality of the volume estimates depended on the surface grid sizes, as will be clear from the discussion below. The method is not new, in that it was suggested in the book by Dierckx [6]. A reader can also consult the Dierckx references [4] and [5].

The report is divided as follows. Section 2 introduces B-splines and tensor products of B-splines. Section 3 describes the surface point generation experiments using the CMM. Section 4 describes the methods and results from applying the spherical and B-spline models to the CMM data. Appendices include the MATLAB codes used in the study.

\section{Introduction to B-splines}

In this section we will define cubic B-splines and show the construction of bivariate or tensor products of B-splines on a two dimensional array. We will then give a discussion of how B-splines are computed. These specific computational algorithms will not be implemented in code, since they are available as functions in the MATLAB spline toolbox. The algorithmic discussions are given, though, as background to understanding the output from the MATLAB functions.

\subsection{Cubic B-Splines in One Variable}

Suppose that a function $y=f(x)$ is known at the $m$ points $\left(x_{1}, y_{1}\right), \cdots,\left(x_{m}, y_{m}\right)$, where $a<x_{1}<$ $x_{2}<\cdots<x_{m}<b, y_{k}=f\left(x_{k}\right)$. It is known that a polynomial of degree $m-1, P(x)$, can be constructed to pass through these $m$ points. In the case of highly accurate data points this polynomial can be constructed to interpolate these points by, for example, Lagrange polynomial or Newton divided difference algorithms. But, in the case of large $m$, it is also well known that 


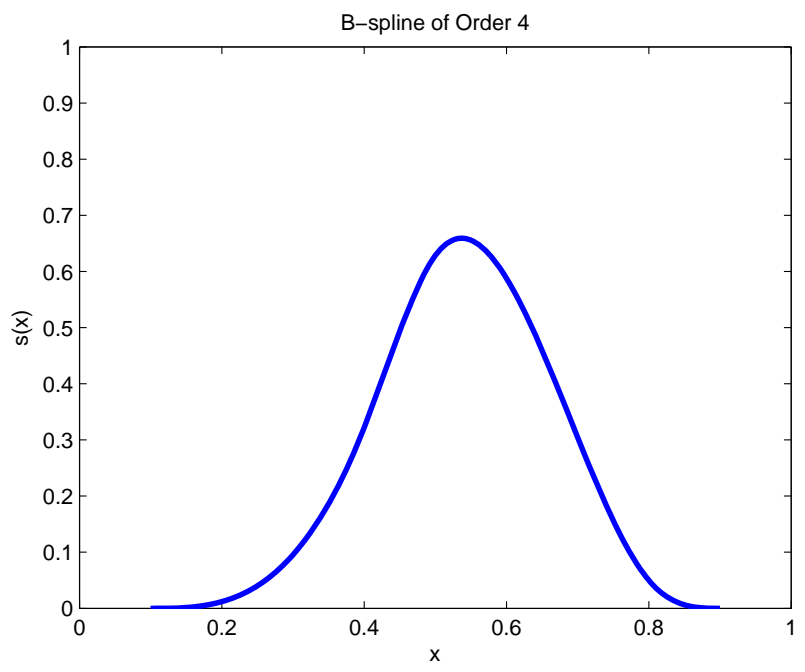

Figure 2: Plot of a B-spline of Order 4.

polynomials of high degree can produce unwanted oscillations between the interpolated points. It is crucial then to approximate sets of data with as low degree polynomials as possible. Of course these polynomials may or may not interpolate the data points but may be made to come as close to them as possible. This is a significant advantage of functions called splines.

Given a set of $m$ real numbers, satisfying $a<x_{1}<x_{2}<\cdots<x_{m}<b$, a spline function, $s(x)$, of order $n$ (or degree $n-1$ ) with knots $x_{1}, x_{2}, \cdots, x_{m}$ is a function that satisfies two properties: (1) in each of the intervals $x \leq x_{1} ; x_{j-1} \leq x \leq x_{j},(j=2,3, \cdots, m) ; x_{m} \leq x, s(x)$ is a polynomial of degree $n-1$ or less. (2) $s(x)$ and its derivatives of orders $1,2, \cdots, n-2$ are continuous. This would mean, for example, a spline function of order four would be constructed from polynomials of degree three (cubic) or less on the intervals $x \leq x_{1} ; x_{j-1} \leq x \leq x_{j},(j=2,3, \cdots, m) ; x_{m} \leq x$ with continuous derivatives of orders one and two. In this report only splines of order four will be used.

Instead of approximating data with one spline, another approach is to build an approximating function by forming a linear combination of functions. These functions are usually referred to as basis functions. This is a well known mathematical technique of constructing complex functions by forming linear combinations of simpler functions. In the current report data sets will be approximated by linear combinations of special spline functions, called B-splines, for Basis splines. Only B-splines of order four will be considered here. B-splines of order four are cubic splines that are non-zero only over four adjacent intervals between knots (see Fig. 2). The notation for a B-spline is $N_{i, k}(x)$, where $N_{i, k}(x)$ is zero everywhere except in the range $x_{i-k}<x<x_{i}$, where in this work $k=4$. As a note, sometimes B-splines are denoted in the literature by $B_{i, k}(x)$, but in this report we will use $N_{i, k}(x)$. To simplify notation let $N_{i}(x)=N_{i, 4}(x)$. Then a B-spline of order four, or cubic B-spline, is a cubic spline with knots $x_{i-4}, x_{i-3}, x_{i-2}, x_{i-1}, x_{i}$ that is zero everywhere except in the range $x_{i-4}<x<x_{i} . N_{i}(x)$ is defined uniquely except for a scaling factor and is conventionally taken to be positive throughout the range $x_{i-4}<x<x_{i}$ and has a single maximum value. Since $N_{i}(x)$ is a cubic spline it has continuous derivatives of order one and two at $x_{i-4}$ and $x_{i}$. These derivatives are zero at the endpoint knots.

To define a complete set of B-splines on the set of points $a<x_{1}<x_{2}<\cdots<x_{m}<b$ it is necessary to introduce eight additional points at the boundaries given by $x_{-3}, x_{-2}, x_{-1}, x_{0}, x_{m+1}$, 
$x_{m+2}, x_{m+3}, x_{m+4}$. It is usual to have $x_{0}=a, x_{m+1}=b$. With this augmented set of knots one can define $m+4$ fundamental cubic B-splines, $N_{i}(x), i=1,2, \cdots, m+4$. Then the general cubic B-spline has a unique representation in the range $a \leq x \leq b$ of the form

$$
s(x)=\sum_{i=1}^{m+4} c_{i} N_{i}(x)
$$

There are computational advantages of cubic B-splines. For any given $x$, all but four adjacent $N_{i}(x)$ are zero. In particular, if $x \in\left[x_{i-1}, x_{i}\right]$ the four non-zero cubic B-splines are $N_{i}(x), N_{i+1}(x), N_{i+2}(x), N_{i+3}(x)$.

A least squares curve fitting problem to $a<u_{1}<u_{2}<\cdots<u_{m}<b$, $y_{k}=f\left(u_{k}\right)$ becomes one of determining the coefficients $c_{i}$ as the least squares solution to the equations

$$
\sum_{i=1}^{m+4} c_{i} N_{i}\left(u_{r}\right)=f_{r}, \quad r=1,2, \cdots, m
$$

These may be written in matrix notation as

$$
A c=f
$$

where $A$ is the $m \times(m+4)$ matrix whose element in column $i$ of row $r$ is $N_{i}\left(x_{r}\right)$ and $c, f$ are column vectors with elements $c_{i}, f_{r}$ respectively. If the data points are arranged in increasing order of $x$, then the matrix $A$ becomes a banded matrix with bandwidth four. For a more thorough discussion of B-splines see de Boor [3].

\subsection{Evaluation of B-splines}

Let $k$ be the order of the desired B-splines and let $x_{1}, x_{2}, \cdots, x_{m}$ be mesh points. In order to develop a complete set of B-splines we need to extend the mesh array by $\mathrm{k}$ points on each end as described in the previous section. On the left end, extend the mesh by setting

$$
x_{-(k-1)}=x_{-(k-2)}=\cdots=x_{0}=a,
$$

and, on the right set

$$
x_{n+1}=x_{n+2}=\cdots=x_{n+k}=b .
$$

Now define a working array that incorporates all of the mesh and the extended mesh points as

$$
\begin{aligned}
x t_{1}=x_{-(k-1)}, x t_{2} & =x_{-(k-2)}, \cdots, x t_{k}=x_{0}, \\
x t_{k+1}=x_{1}, x t_{k+2} & =x_{2}, \cdots, x t_{n+k}=x_{n}, \\
x t_{n+k+1}=x_{n+1}, x t_{n+k+2} & =x_{n+2}, \cdots, x t_{n+2 k}=x_{n+k} .
\end{aligned}
$$

In this section the double subscript for B-splines will be used since it is needed to define the computational algorithm. The B-splines, $N_{i k}(x)$, are constructed by a recursion formula given by

$$
N_{1 i}(x)= \begin{cases}1 & \text { if } x_{i} \leq x \leq x_{i+1} \\ 0 & \text { otherwise }\end{cases}
$$

and

$$
N_{k i}(x)=\frac{\left(x-x_{i}\right) N_{k-1, i}(x)}{x_{i+k-1}-x_{i}}+\frac{\left(x_{i+k}-x\right) N_{k-1, i+1}(x)}{x_{i+k}-x_{i+1}} .
$$


This formula is referred to in the literature as the Cox-deBoor formula (see Cox [2]).

To evaluate a B-spline of order $k$ for a given $t \in\left[x_{1}, x_{n}\right]$ the first step is to find $i \in[k+1, n+k]$ so that $t \in\left[x t_{i}, x t_{i+1}\right]$. Note that if $t=x_{n}$ then $i=n+k$. Begin by initializing the array $N(j, i)=0$ for $i=1, \ldots, n+k, j=1, \cdots, k$. By the Cox-deBoor formula set $N(1, i)=1, N(1, j)=0, j \neq i$ and

$$
N(j, i)=\frac{(t-x t(i)) N(j-1, i)}{x t(i+j-1)-x t(i)}+\frac{(x t(i+j)-t) N(j-1, i+1)}{x t(i+j)-x t(i+1)} .
$$

In any implementation of this formula care must be exerted due to the potential division by zero problem in the coefficients. We have dropped the function representation here and chosen an array form for the ease of algorithmic representattion.

Once an interval index $i$ has been identified with a given $t$, the evaluation of the final B-spline begins by setting $N(1, i)=1$. It will happen that only $k$ values $N(k, i-k+1), N(k, i-k+$ $2), \cdots, N(k, i)$ will be nonzero. This occurs since there is a triangular dependency of B-splines as

$$
\begin{array}{cccc} 
& & & N(1, i) \\
& N(3, i-2) & N(2, i-1) & N(2, i) \\
& \ldots & \ldots & N(3, i) \\
N(k, i-k+1) & \cdots & \cdots & N(k, i)
\end{array}
$$

From the discussion here it will become clear why the computations for the relevant B-splines can be reduced to computing (10). In fact, the computation of the B-splines along the right leg of the triangle depends only on the B-splines just above on the right leg. The computation of the $\mathrm{B}$-splines along the hypotenuse of the triangle depends only on the B-splines directly to the right and above along the hypotenuse. Finally, the B-splines within the triangle depend on the B-splines just above and to the right. This can easily be seen from the Cox-deBoor formula and (10). First of all $N(1, i)=1$ initializes the recursion. By Cox-deBoor, $N(2, i)$ depends on $N(1, i)$ and $N(1, i+1)$, but $N(1, i+1)=0$, so $N(2, i)$ depends only on $N(1, i)$. Now $N(2, i-1)$ depends on $N(1, i-1)$ and $N(1, i)$, but $N(1, i-1)=0$, so $N(2, i-1)$ depends only on $N(1, i-1)$. Now consider $N(3, i)$. This depends on $N(2, i)$ and $N(2, i+1)$, but $N(2, i+1)$ depends on $N(1, i+1)$ and $N(1, i+2)$, which are both zero. So $N(3, i)$ depends only on $N(2, i)$. $N(3, i-1)$ depends, by Cox-deBoor, on $N(2, i-1)$ and $N(2, i)$, which are both nonzero. Finally, $N(3, i-2)$ depends on $N(2, i-2)$ and $N(2, i-1)$, but $N(2, i-2)=0$ since it depends on $N(1, i-2)=0$ and $N(1, i-1)=0$. This argument can easily be extended down the triangle, but the pattern is clear. Any implementation of the Cox-deBoor formula can then be done by proceeding down the right leg, then down the hypotenuse and then fill in the interior of the triangle.

There is a property of B-splines that can be used to check the calculation of the final $k$ nonzero B-splines. It is called a "partition of unity" property in that

$$
\sum_{j=i-k+1}^{i} N(k, j)=1 .
$$

\subsection{Tensor Products of Cubic B-splines in Two Variables}

In Section 2.1 the problem of least squares approximation of data by one dimensional B-splines was discussed. In this section the B-spline concept will be extended to two dimensions in order to fit two dimensionsl scattered data by a surface function. In this surface-fitting problem data points $\left(u_{r}, v_{r}\right), r=1,2, \cdots, k$ and values at these points, $z_{r}=f\left(u_{r}, v_{r}\right), r=1,2, \cdots, k$ are given. The surface model used to fit these data points will involve sums of products of B-splines. 
To introduce this model, define a rectangle, say $R$, by $a \leq x \leq b, c \leq y \leq d$ in the $(x, y)$ plane. The definition does not restrict the data points to the Euclidean plane. They could, for example be angular coordinates, as will be seen later in this report. The rectangle is subdivided by sets of knots $x_{i}, i=1,2, \cdots, m$ and $y_{j}, j=1,2, \cdots, n$, where $a<x_{1}<x_{2}<\cdots<x_{m}<b$ and $c<y_{1}<y_{2}<\cdots<y_{n}<b$. These knots divide the rectangle $R$ into rectangular panels in the plane given by $R_{i j}, i=1,2, \cdots, m, j=1,2, \cdots, n$. Then, after extending the knots by eight in each dimension as done in the one dimensionsl case, a basis set of splines for this pairing of coordinates can be constructed as products of B-splines $N_{i}(x) N_{j}(y)$. In fact the surface spline model will be given by

$$
s(x, y)=\sum_{i=1}^{m+4} \sum_{j=1}^{n+4} c_{i j} N_{i}(x) N_{j}(y)
$$

called a tensor product of splines.

As in the one dimensional case these tensor product splines have a number of advantages. First of all, the basis functions $N_{i}(x) N_{j}(y)$ are each non-zero only over a rectangle composed of sixteen panels in a $4 \times 4$ arrangement. In particular $N_{i}(x) N_{j}(y)$ is non-zero only when $x_{i-4} \leq x \leq x_{i}$ and $y_{j-4} \leq y \leq y_{j}$. Next, if $\left(u_{r}, v_{r}\right), r=1,2, \cdots, m$ and values at these points, $z_{r}=f\left(u_{r}, v_{r}\right), r=$ $1,2, \cdots, m$ are given, then the least squares fitting problem can be formulated as finding the least squares solution of

$$
\sum_{i=1}^{m+4} \sum_{j=1}^{n+4} c_{i j} N_{i}\left(u_{r}\right) N_{j}\left(v_{r}\right)=z_{r}, r=1,2, \cdots, k .
$$

Again, this can be written in a matrix form as

$$
A c=f,
$$

where $\mathrm{A}$ is now a matrix with $k$ rows and $(m+4)(n+4)$ columns. If the indexing has been arranged so that $N_{i}(x) N_{j}(y)$ and the corresponding $c_{i j}$ are arranged in the order for fixed $j$, with $i=1,2, \cdots, m+4$, where $j=1,2, \cdots, n+4$, then $A$ has as its element $N_{i}\left(u_{r}\right) N_{j}\left(v_{r}\right)$ in row $r$ of column $(j-1)(m+4)+i$. If the data points are also arranged according to the panels $R_{i j}$ containing them in the order for fixed $j, i=1,2, \cdots, m+4$, where $j=1,2, \cdots, n+4$, then the matrix $A$ takes the form

$$
A=\left[\begin{array}{ccccccc}
A_{11} & A_{12} & A_{13} & A_{14} & & & \\
& A_{22} & A_{23} & A_{24} & A_{25} & & \\
& & A_{33} & A_{34} & A_{35} & A_{45} & \\
& & \ddots & \ddots & \ddots & \ddots & \\
& & & A_{n+1, n+1} & A_{n+1, n+2} & A_{n+1, n+3} & A_{n+1, n+4}
\end{array}\right] .
$$

Here each $A_{r s}$ is a rectangular matrix of bandwidth four with $m+4$ columns and the number of rows is equal to the total number of data points in the panels $R_{1 r}, R_{2 r}, \cdots, R_{n+1, r}$. For a full discussion of tensor products of B-splines see de Boor [3], Eberly [8], and Rogers and Adams [13].

\subsection{Issues in Computing Tensor Product B-splines and the Relation to Least Squares}

Unfortunately this block format for the matrix $A$ is only achievable if the knots, defining the Bsplines in the separate dimensions, can be ordered properly. If, for example, two dimensional points are dependent, as in a set of coordinate points $\left(x_{i}, y_{i}\right), i=1, \cdots, n$ on a surface, it may not be 
possible to order the variables as described above. In that case, the resulting tensor product matrix might be rank deficient and it will not be possible to use the traditional normal equations to solve a least squares problem. Another approach to solving the least squares problem in this case will be discussed below.

In this section, though, we will first discuss the MATLAB function tensor_prod_spl that is given in Appendix C.4. This function creates the tensor product matrix that will be called NM. The input to this function includes the set of knots for both the $\theta$ and $\phi$ angular dimensions as well as the vector arrays of $\theta$ and $\phi$ variables at which the tensor product of $\mathrm{B}$-splines is to be evaluated.

The first thing done for each dimension is to augment the knots at each end. This is done by calls to the MATLAB spline toolbox function augknt, where the order of the B-splines has been set to four for cubic B-splines. This function is called for each of the two knot sets. The matrix for each B-spline, $\mathrm{N}$ for $\theta$ and $\mathrm{M}$ for $\phi$, is then created by a call to the MATLAB spline toolbox function spcol. This function can create the entire B-spline matrix for a vector of variables, provided the vector is ordered in ascending order. This is possible if the variables in both dimensions are independent. But in the case of coordinates, the variable arrays are not independent. In this case, although one dimension can be sorted in ascending order, the other one has to be ordered in such a way that the coordinates are always linked. Thus, although the $\theta$ could be sorted, the $\phi$ array would not necessarily be sorted properly. Therefore the approach taken in the program was to create the individual B-spline matrices one row at a time.

Once the $\mathrm{N}$ and $\mathrm{M}$ matrices are created for the $\theta$ and $\phi$ arrays, the tensor product matrix NM can be created. This is done by taking all possible products of the elements from the $\mathrm{N}$ and $\mathrm{M}$ matrices.

Once the tensor product B-spline matrix is computed, a least squares fit can be done to the scattered spherical coordinate data. Since the matrix NM could be rank deficient, with potentially zero rows or columns, we cannot rely on the standard normal equations for determining the coefficients. We will use the singular value decomposition (SVD) in this case. This is done in the MATLAB function lsq_2D_spline in Appendix C.5. The input to this function is the tensor product matrix $\mathrm{NM}$ and the array of radial values at the scattered spherical coordinate points, along with a desired data tolerance. The data tolerance is used to zero the small singular values. In this function the matrix NM, with the number of rows larger than the number of columns, is decomposed by the MATLAB function svd into the product of a column-orthogonal matrix $U$, a diagonal matrix $S$, and the transpose $V^{\prime}$ of a square orthogonal matrix, so that $\mathrm{NM}=U S V^{\prime}$. In order to solve the problem NMc $=\mathrm{r}$ we compute c $=V S^{+} U^{\prime} r$, where $S^{+}$is the generalized inverse of $S$ and $U^{\prime}$ is the transpose of $U$.

\subsection{A Knot Selection Algorithm}

The selection of B-spline knots for an optimum fit is a nontrivial task. One would be extremely lucky to manually select an initial set of knots and achieve an optimum fit. Therefore we approach knot selection in an iterative fashion that moves us toward an optimum selection. The overall strategy is to place knots in the vicinity of the fit where the local residuals are the largest. This process involves not only the placement algorithm issue but also the iterative stopping criteria.

First we will discuss the placement algorithm. It is based on a suggested strategy by Dierckx [6]. At the beginning of each iteration the assumption is that there exists a current set of knots, call them knts_theta and knts_phi. In the first iteration of the algorithm these would be an initial set chosen by the user. The tensor product of the B-splines are computed at the data points, a least squares fit is made to the data, and the current absolute residuals of the fit are computed at each data point. The current knots divide the $\theta, \phi$ plane into rectangles. Some rectangles have data points and others 
don't. For each i from 1 to the number of data points minus 1 the array indices of $\theta$ are found so that knts_theta(i) $<=$ theta $<=$ knts_theta(i+1). The sums of the residuals at these points are computed and the maximum of these sums is chosen. A similar calculation is done for $\phi$ and the maximum of the sums of the squares of the residuals is chosen. The maximum of these two maximums is chosen. If the maximum in the $\theta$ dimension is chosen then a knot is added to the knts_theta array, otherwise a knot is added to the knts_phi array. The algorithm steps are given below. For the sake of argument assume that there are currently $g \theta$ knots and $h \phi$ knots that form a grid defined by the rectangles $[$ knts_theta(i), knts_theta(i +1$)] \times\left[\right.$ knts_phi $\left.(\mathrm{j}), \operatorname{knts} \_p h i(\mathrm{j}+1)\right], i=1, \cdots, g-1, j=1, \cdots, h-1$. Here is the placement algorithm used.

$$
\begin{aligned}
R_{k} & =r_{k}-S\left(\theta_{k}, \phi_{k}\right), k=1, \cdots, n \\
\delta_{i}^{\theta} & =\sum_{k}\left\{R_{k}^{2}: \operatorname{knts} \_t h e t a(\mathrm{i}) \leq \theta(k) \leq \operatorname{knts\_ theta}(\mathrm{i}+1)\right\} \\
\delta_{j}^{\phi} & =\sum_{k}\left\{R_{k}^{2}: \operatorname{knts} \_t h e t a(\mathrm{i}) \leq \theta(k) \leq \operatorname{knts\_ theta}(\mathrm{i}+1)\right\} \\
\delta^{\theta} & =\max _{1 \leq i \leq g-1} \delta_{i}^{\theta} \\
\delta^{\phi} & =\max _{1 \leq j \leq h-1} \delta_{j}^{\phi} \\
\text { new_knt_theta } & =\sum_{k}\left\{R_{k}^{2} \theta(k) / \delta^{\theta}\right\}, \quad \text { if } \delta^{\theta} \geq \delta^{\phi} \\
\text { new_knt_phi } & =\sum_{k}\left\{R_{k}^{2} \phi(k) / \delta^{\phi}\right\}, \quad \text { if } \delta^{\phi} \geq \delta^{\theta}
\end{aligned}
$$

The stopping criteria used in this algorithm is based on the use of the $R^{2}$-statistic, called the coefficient of multiple determination (see Draper and Smith [7]). $R^{2}$ is the square of the correlation between the vector of observed data, $r_{k}, k=1, \cdots, n$, and the least squares predicted data, $\hat{r}_{k}, k=$ $1, \cdots, n$. The statistic satisfies $0 \leq R^{2} \leq 1$. This statistic is often used as a measure of how well the regression equation explains the variation in the data. It is known that building models based on adding terms in the regression equation, care must be taken in using this statistic. However, in the current algorithm the statistic is used in a somewhat non-conventional manner. We use it as a measure of the benefit of adding more knots to the tensor product spline model. The knot selection algorithm is terminated once $R^{2}>0.98$.

\section{Surface Point Data Generation}

In this section we discuss the experimental method by which boundary data was generated in Euclidean coordinates on each of the phantoms. Figure 3 shows the CMM system used to measure the phantoms and a calibrated sphere used to check the probe. The system is computer controlled and touches an object to be measured at programmend points in order to produce $(x, y, z)$ values at the probed points.

The effective diameter of the CMM probe was measured by first measuring the high quality steel sphere, with a well-calibrated diameter. This sphere is separate from the calibrated one used to generate the sphere data in Section 4.2.1 below. The calibration sphere can be seen in the background of Fig. 4. The difference between the known diameter and the apparent diameter of the measured referrence metal sphere gave the calibrated effective probe diameter. A similar pattern of probe touches was used for the measurement of the calibrated sphere and for all of the phantom sphere measurements. 


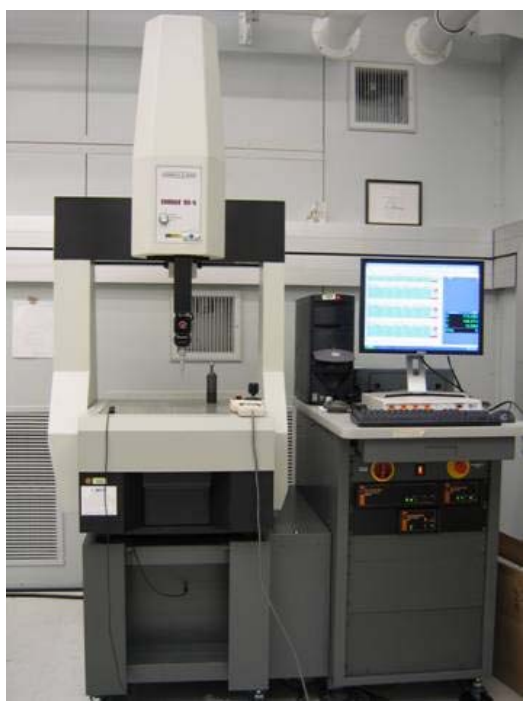

Figure 3: Computer controlled CMM.

The FDA phantoms were created by a molding process and the mold marks on both spheres were visible. These mold marks were used to align the phantoms with the coordinate system of the CMM. The marks were laid out like lines of latitude, leading to the use of a natural nomenclature of latitude and longitude like those of the Earth. For each phantom, the north pole was chosen to be the one with darker, deeper, or more obvious mold marks.

The phantoms were held by a vacuum chuck (see Fig. 4) to minimize distortion and reduce the chance of damaging the spheres' surfaces. The chuck has a shallow cone, so contact is made around a circle of latitude at about $-45^{\circ}$. The wall vacuum was strong enough to hold the spheres sufficiently that they did not move significantly, as shown by the repeatable results from run to run at the 1 $\mu \mathrm{m}$ level.

Visually, the pink sphere was noticeably out of round, in the shape of an oblate spheroid. A dial caliper gave diameter measurements given in Table 1.

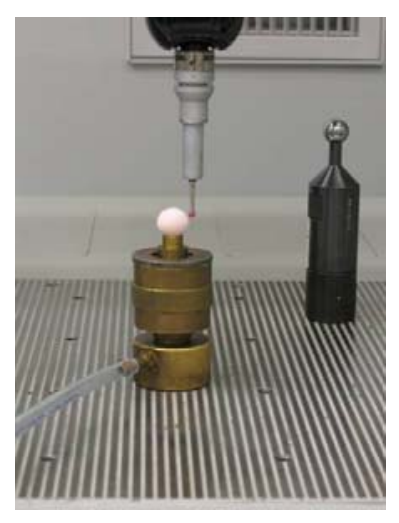

Figure 4: Lung nodule phantom on the vacuum chuck. 


\begin{tabular}{|l|c|c|}
\hline \multirow{2}{*}{ Pink } & Equatorial & Polar \\
\cline { 2 - 3 } & $20.0 \mathrm{~mm}$ & $18.4 \mathrm{~mm}$ \\
\hline Green & $20.0 \mathrm{~mm}$ & $20.1 \mathrm{~mm}$ \\
\hline
\end{tabular}

Table 1: Dial Caliper Measurements of Phantoms' Diameters

The uncertainty of caliper measurements on hard steel surfaces is about $0.1 \mathrm{~mm}$, and is estimated to be about $0.3 \mathrm{~mm}$ on the sample spheres due to the potential that the contact force would distort the soft surfaces of the spheres (all estimated uncertainties were $\mathrm{k}=2$ expanded uncertainties according to the guidelines of Taylor and Kuyatt [18]). Since the CMM is primarily used to measure metallic artifacts produced by machining operations, we will see in this report some of the possible consequences of using the CMM to measure the non-metallic phantoms.

Two probing experiments were performed on each of the phantoms and the calibrated sphere. They created what we will call a coarse data set and a dense data set. In the next two sections we will discuss the methods used in the two experiments since they were slightly different.

\subsection{Experiment 1: Coarse Data Set}

In this experiment the plan was to measure each phantom on the CMM three times in each position, with 61 coordinate points per hemisphere. For the green sphere, each measurement set consisted of three separate sets of points: North pole up, South pole up, and prime meridian/equator intersection up. The pink sphere could not be held sideways in the first experiment, as the out-of-roundness prevented an effective vacuum seal. Therefore, a measurement data set for this sphere had only the North pole up and South pole up data.

Although 183 points were measured on the green sphere, 121 were selected in order to make the data sets for the green and pink phantoms consistent in terms of probed points. All 121 points from both hemispheres were merged together, and the plots in Fig. 5 and 6 show the radial deviation from a best-fit sphere for the full data sets. The figures show the radial residuals obtained by fitting sphere models to the Green and Pink data with an algorithm ordinarily used during sphere calibration work. In particular, they represent the residual errors between the distance from the fitted sphere center to the probed points and the fitted radius of the sphere model. The residuals, in the case of the Green phantom, range from approximately $-0.1 \mathrm{~mm}$ to $+0.1 \mathrm{~mm}$, whereas the residuals, in the case of the Pink phantom, range from approximately $-0.57 \mathrm{~mm}$ to $+0.23 \mathrm{~mm}$. This indicates the non-spherical nature of the Pink phantom. A separate nonlinear algorithm for sphere fitting will be described in Section 4. It produces consistent results. Figure 7 shows the typical distribution of the probe points on a sphere. The plot is a transparency so that probe points on the opposite side of the sphere are visible. The calibated sphere on the shaft, with a diameter of 19.05 $\mathrm{mm}$, was also measured with 121 points, repeated five times.

\subsection{Experiment 2: Dense Data Set}

In this experiment 181 points were taken on the phantoms and the calibrated sphere, with five repeats in each position. The positions were taken the same as those in Experiment 1. That is, the alignment was selected with North pole up (position 1), South pole up (position 2), and prime meridian/equator intersection up (position 3). In this case it was possible to hold the Pink phantom in the sidways position 3 . The calibrated sphere was measured in only one position, with five repeats, since it was permanently mounted on a support shaft. 


\section{Green Sphere Residuals}

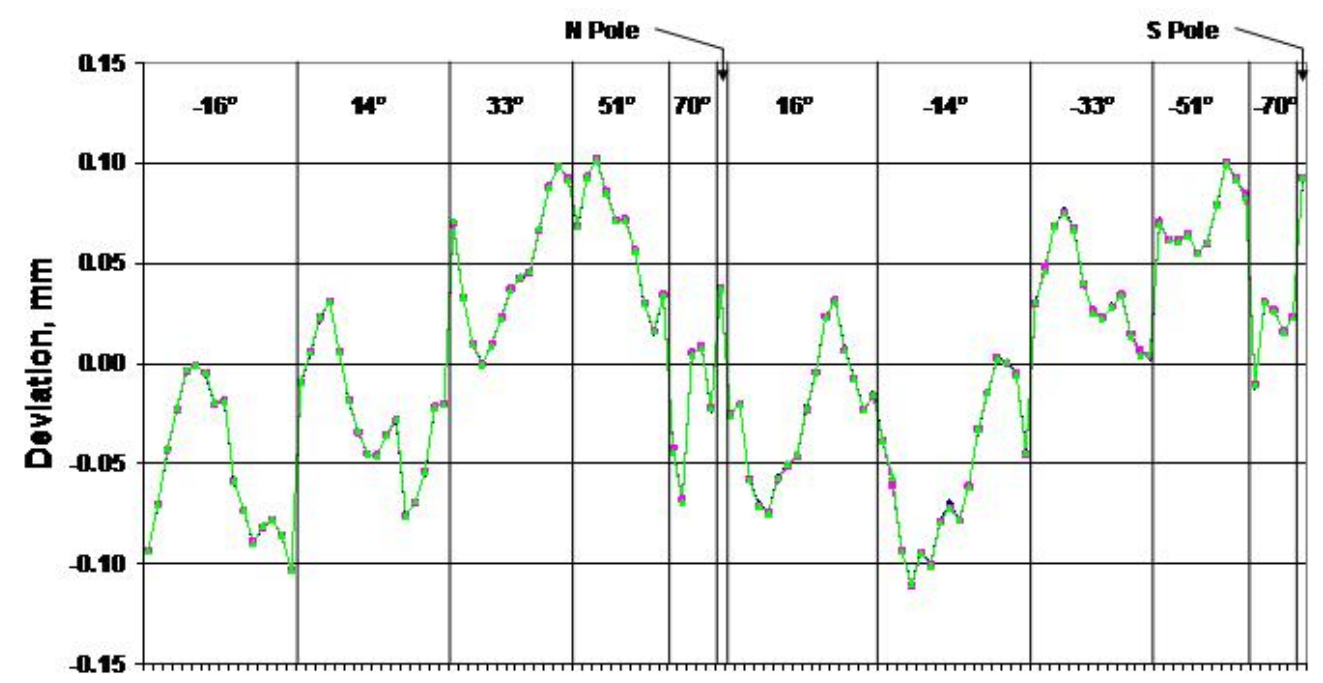

Points Grouped by Latitude

$\rightarrow$ Run $1 \rightarrow-$ Run $2 \rightarrow-$ Run 3

Figure 5: Residual Measure CMM Errors for the Green Phantom Sphere: Experiment 1.

\section{Pink Sphere Residuals}

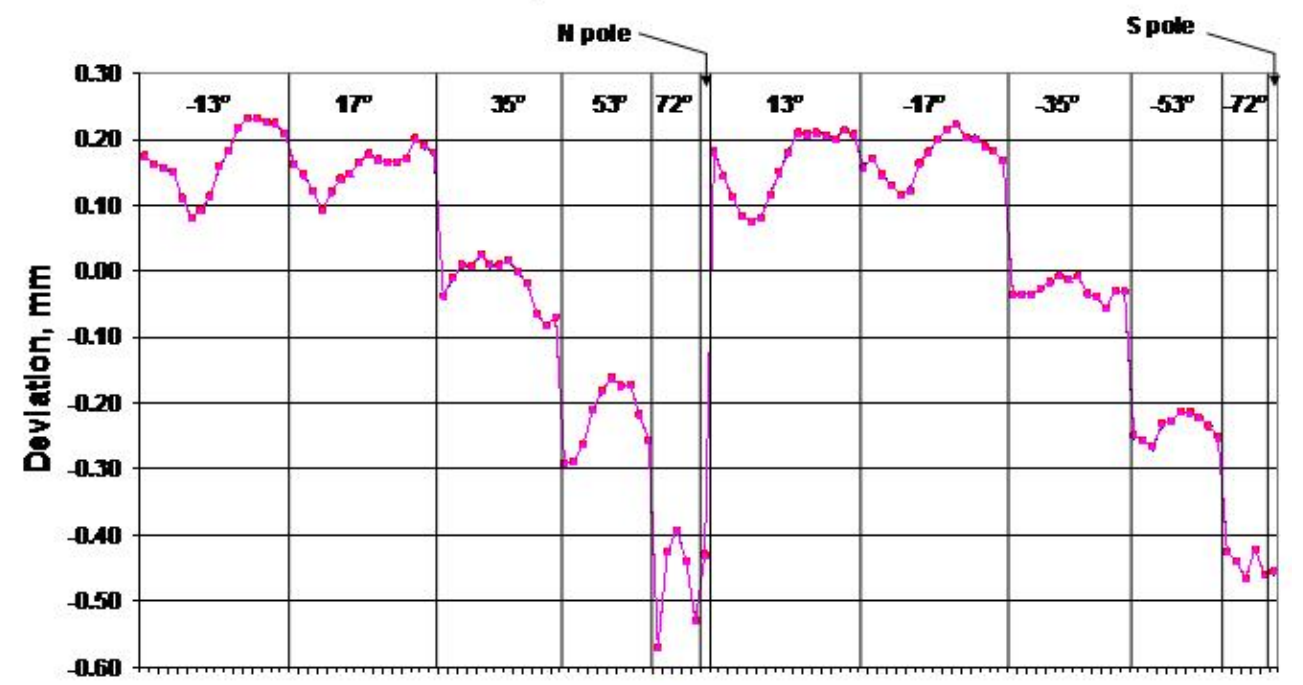

Points Grouped by Latitude

$\rightarrow$ Run $1 \rightarrow-$ Run $2 \rightarrow$ Run 3

Figure 6: Residual Measure CMM Errors for the Pink Phantom Sphere: Experiment 1. 


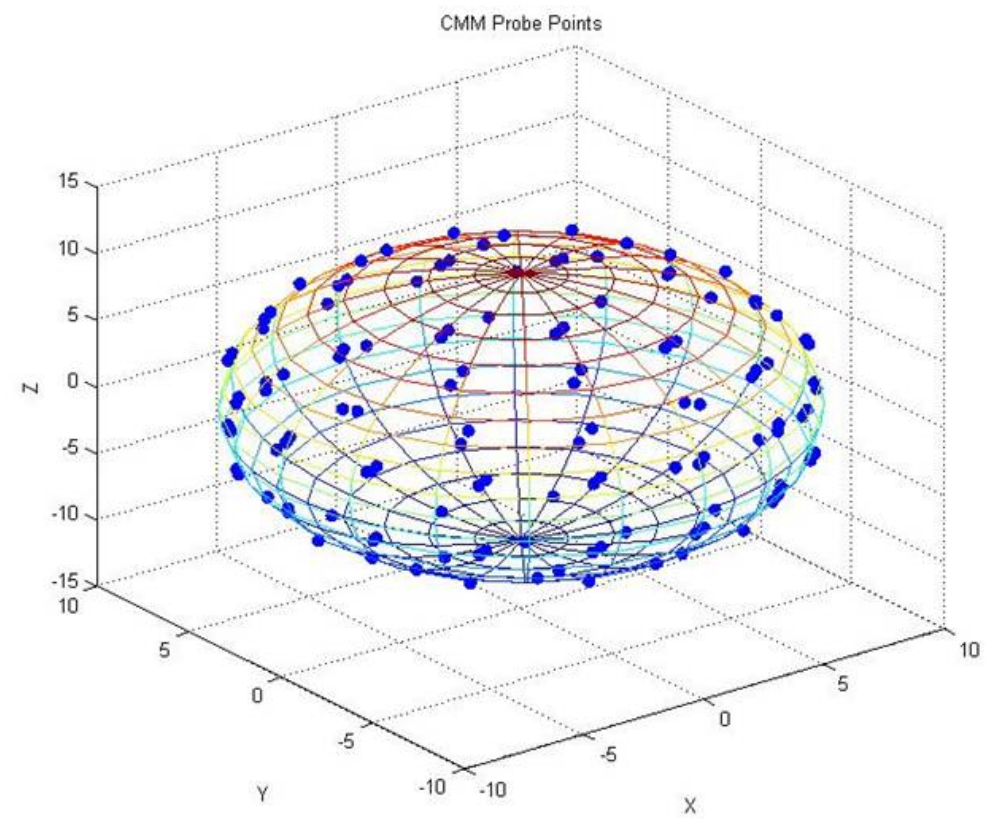

Figure 7: Distribution of the CMM Probe Points on a Sphere: Experiment 1.

\section{Data Modeling and Volume Estimation}

In this section two forms of data modeling will be discussed. Since the phantoms seemed to be nearly spherical the natural tendency was to first consider fitting a spherical model to each phantom and estimating the volume of the fitted spheres. Second, in order to develop a potentially more accurate volume estimation model the surface data was fit using tensor products of B-splines and the volumes estimated by the Divergence Theorem.

\subsection{A Spherical Model}

Since the phantoms were nearly spherical it was natural, as noted above, to consider how close the data could be modeled by first assuming spherical models for the data. The calibrated sphere, of course, could clearly be modeled with a spherical model.

In particular, let $\left.c=\left(c_{1}, c_{2}, c_{3}, c_{4}\right)\right)$ and set

$$
f(x, y, z, c)=\left(x-c_{1}\right)^{2}+\left(y-c_{2}\right)^{2}+\left(z-c_{3}\right)^{2}-c_{4}{ }^{2} .
$$

The unknown parameters $c_{1}, c_{2}, c_{3}$ represent the center of the sphere and $c_{4}$ is the radius. All of the data were measured in millimeters so that the parameters naturally have millimeter units. Define

$$
S(c)=\sum_{i=1}^{n}\left\{\left(x_{i}-c_{1}\right)^{2}+\left(y_{i}-c_{2}\right)^{2}+\left(z_{i}-c_{3}\right)^{2}-c_{4}^{2}\right\} .
$$

Since the function $S$ is a nonlinear implicit function of the parameters we needed to use a nonlinear 
mimimization algorithm to solve the problem

$$
\min _{c} S(c)
$$

There are a number of algorithms for fitting least squares models to data on geometric shapes. The reader can consult Shakarji [16]. There are also various algorithms for minimizing general nonlinear functions, such as (18). All of the algorithms involve iterative minimization of some form. Many require computing derivatives of the objective function in order to generate minimization search directions. Others do not involve derivatives but may be somewhat slower in the minimization search. The algorithm selected here, because of the relatively few parameters involved, and the fact that derivatives are not required, is a form of polygon search method called the Nelder-Mead method (see Sauer [14]). It should be noted here that the Newton's method, requiring derivatives, was initially used to estimate the parameters, but the Nelder-Mead tended to produce the smallest value to (18).

The Nelder-Mead method begins with an initial vector guess for the minimum in $R^{4}$, although the method works for $R^{n}$, but due to the "curse of dimension", becomes prohibitive beyond $R^{10}$. From the initial vector guess a polygon of five vertices is built. The function evaluations at the vertices, $w_{i}=S\left(v_{i}\right)$, of the polygon are tested and put into ascending order, $w_{1}<w_{2}<\cdots<w_{5}=w_{h}$. The polygon vertex, $v_{h}$, with the largest function value, $w_{h}$, is replaced as follows. The centroid, $\bar{v}$, of the face of the polygon that does not contain $v_{h}$ is chosen. The function (18) is evaluated at, $v_{r}$, the reflection point about the centroid, given by $v_{r}=2 \bar{v}-v_{h}$, is selected. If the value $w_{r}=S\left(v_{r}\right)$ lies in the range $w_{1}<w_{r}<w_{4}$, then $v_{r}$ replaces $v_{h}$, the function values of the vertices are again sorted, and the iterative step is repeated. If $w_{r}<w_{1}$, extrapolation is continued in the direction of $w_{r}$ by setting $v_{e}=3 \bar{v}-2 v_{h}$. The better of $v_{e}$ or $v_{r}$ is selected to replace $v_{h}$. Finally, if $w_{r}>w_{n}$ then a contraction of points is attempted. Two points are selected. The outer contraction point is taken as $v_{o c}=1.5 \bar{v}-0.5 v_{r}$ and the inner contraction is $v_{i c}=0.5 \bar{v}+0.5 v_{h}$. The function (18) is evaluated at both contraction points. If there is no reduction in the value of (18) then the polygon is shrunk by a factor of 2 in the direction of the current minimum, $v_{1}$. The iterations of sorting and testing continue until the volume of the polygon becomes less than some prespecified tolerance. Given a set of $(x, y, z)$ points, a MATLAB code to estimate the center and radius of the best fit sphere is given in Appendix A. The output of the program produces a vector that is the median value of the vertex values of the final polyhedron, whose volume satisfies the tolerance specification. The volume is computed as a determinant involving the polygon vertices.

The uncertainties of the estimated center and radius were computed using the methods proposed in Draper and Smith [7] for nonlinear regression. In particular, if $\hat{c}=\left(\hat{c}_{1}, \hat{c}_{2}, \hat{c}_{3}, \hat{c}_{4}\right)$, then define the matrix with elements

$$
\hat{Z}_{i j}=\frac{\partial f}{\partial c_{i}}\left(x_{i}, y_{i}, z_{i}, \hat{c}\right), \quad i=1, \cdots, n, \quad j=1,2,3,4 .
$$

The matrix $\hat{Z}$ is an $n \times 4$ matrix. The $i$-th row is given by

$$
\hat{Z}_{i}=\left[\begin{array}{llll}
-2\left(x_{i}-\hat{c}_{1}\right) & -2\left(y_{i}-\hat{c}_{2}\right) & -2\left(z_{i}-\hat{c}_{3}\right) & -2 \hat{c}_{4}
\end{array}\right] .
$$

If we then form $\hat{Z}^{\prime} \hat{Z}$ the standard error of $\hat{c}_{i}$, s.e. $\left(\hat{c}_{i}\right)$, is given by the $i$-th diagonal element of $\left(\hat{Z}^{\prime} \hat{Z}\right)^{-1} s^{2}$ where $s=S(\hat{c}) /(n-4)$. The expanded uncertainty is given by 2 s.e. $\left(\hat{c}_{i}\right)$ as defined in Taylor and Kuyatt [18]. The uncertainty limits of $\hat{c}_{i}$ are $\hat{c}_{i} \pm 2$ s.e. $\left(\hat{c}_{i}\right)$. All units are in millimeters except volume, which is in cubic millimeters. 


\subsection{Computational Results for the Spherical Model}

Tables 2, 3, and 4 present the results obtained by fitting spherical models to the data from the calibrated sphere and the two phantoms. In Experiment 1 there were three data sets for each of the Green and Pink phantoms, designated Green 4, 5, and 6 and Pink 5, 6, and 7. Since the output of the Nelder-Mead algorithm was a final polytope surrounding the minimum with the polytope vertices representing the final parameter esimates, the final reported parameters were selected as the median values of the vertex values. These are the first four entries in the table: Center $\mathrm{x}$, Center $y$, Center z, and Radius. The units are millimeters. The fifth table entry is the spherical volume based on the median radius value in cubic millimeters. The radius residuals were computed as

$$
\operatorname{resid}_{i}=\sqrt{\left(x_{i}-\hat{c}_{1}\right)^{2}+\left(y_{i}-\hat{c}_{2}\right)^{2}+\left(z_{i}-\hat{c}_{3}\right)^{2}}-\hat{c}_{4} .
$$

The sixth entry in the tables gives the mean value of these residuals. The unit is millimeters. The seventh and eighth table entries give the mean and standard deviation of the absolute values of the residuals in millimeters. The ninth through the twelfth entries are the expanded uncertainties for the estimated Center x, Center y, Center z, and radius.

The results in these sections on spherical model fitting involve strictly fitting the non-linear model (22) to the sparse data sets generated by the CMM. This process does not involve the Bspline algorithm used later in this report. Therefore any differences between coarse and dense results are most likely the consequence of the point selection in the metrology of the artifacts.

\subsubsection{Results for the Spherical Model Fit to the Calibrated Sphere with Coarse and Dense Data}

Table 2 gives the results of the fit of the sphere model to both the coarse (121 points) and the dense (181 points) data sets for the calibrated sphere. In both cases the average of the five repeat data sets was used for the fitting process. As can be seen, the two volume estimates differ by approximately $0.04 \%$. The radii estimates differ by about $0.01 \%$. It would seem that these differences are within the operating regime of the CMM. Although the estimated centers are slightly different, all other values are of the same order of magnitude. Based on these results we can accept that the CMM is producing accurate position data for spherical metallic artifacts. Tables 3 through 6 , however, begin to show the consequences involved with attempting to measure slightly non-spherical and non-metallic artifacts with the CMM.

\subsubsection{Results for the Spherical Model Fit to the Phantoms with Coarse Data}

From Tables 3 and 4 it is clear that the Green phantom is more spherical than the Pink phantom as indicated by the residuals and the expanded uncertainties. This simply confirms the fact that the Pink phantom was more difficult to measure using the vacuum chuck due to its lack of sphericity. For example, the Mean Radial Residual for the Pink data is two orders of magnitude larger than for the Green data. The Standard Deviations for the Pink data are an order of magnitude greater, whereas all of the Expanded Uncertainties are two orders of magnitude greater. It is not clear how much the phantom material affected the results since it was difficult to set up a specific probe test for metallic versus phantom material. The artifacts would have to have been exactly the same size, positioned at exactly the right location, and probed at exactly the same coordinates to separate those factors from the material factor difference. There were no such comparable artifacts. We can make a guess, though, that there might be some affect due to probe force against the non-metalic material if we look at Table 2 and Table 3, the most spherical of the two phantoms. The Expanded Uncertainties 


\begin{tabular}{||c|c|c||}
\hline \hline \multicolumn{3}{|c|}{ Spherical Fit Results for Calibrated Sphere } \\
\hline Properties & Coarse & Dense \\
\hline \hline Center x & $0.2803 \times 10^{-4}$ & $-0.1331 \times 10^{-2}$ \\
\hline Center y & $-0.4881 \times 10^{-3}$ & $-0.2034 \times 10^{-2}$ \\
\hline Center z & $-0.6642 \times 10^{-2}$ & $-0.2564 \times 10^{-2}$ \\
\hline Radius & 9.5326 & 9.5314 \\
\hline Est. Volume & 3628.4 & 3627.1 \\
\hline Mean Rad. Residual & $0.3232 \times 10^{-4}$ & $0.8720 \times 10^{-4}$ \\
\hline Mean Abs. Rad. Residual & $0.1646 \times 10^{-2}$ & $0.1700 \times 10^{-2}$ \\
\hline Stand. Dev. Rad. Residual & $0.2156 \times 10^{-2}$ & $0.22302 \times 10^{-2}$ \\
\hline Stand. Dev. Abs. Rad. Residual & $0.1384 \times 10^{-2}$ & $0.1441 \times 10^{-2}$ \\
\hline Expanded Uncert. Center x & $0.3676 \times 10^{-9}$ & $0.2779 \times 10^{-9}$ \\
\hline Expanded Uncert. Center y & $0.3675 \times 10^{-9}$ & $0.2777 \times 10^{-9}$ \\
\hline Expanded Uncert. Center $\mathrm{z}$ & $0.9313 \times 10^{-9}$ & $0.7059 \times 10^{-9}$ \\
\hline Expanded Uncert. Radius & $0.2394 \times 10^{-9}$ & $0.1822 \times 10^{-9}$ \\
\hline \hline
\end{tabular}

Table 2: Results of a Spherical Fit to Coarse and Dense Data for Calibrated Sphere

for the sphere fit to the calibrated metalic sphere and the Expanded Uncertainties for the sphere fit to the Green phantom data differ by five to six orders of magnitude. Since the repeatability of the CMM measurements is at the $1 \mu \mathrm{m}$ level, it is likely then that material difference had some significant affect on the difference in the uncertainties. It is a guess that this and the non-spherical shape of the Pink phantom account for a large part of the differences between the Pink phantom Expanded Uncertainties in Table 4, the Green phantom Expanded Uncertainties in Table 3, and the calibrated sphere Expanded Uncertainties in Table 2.

\subsubsection{Results for the Spherical Model Fit to the Phantoms with Dense Data}

We want to make some observations about the resulting measurements and the spherical fits to the dense data. If we first look at the residuals for the sphere fits to the dense data sets we see an interesting phenomenon occuring in the fits for both the Green and Pink phantoms in position 3 (prime meridian/equator intersection up). There appears to be a definite periodic oscillation in the residual data in Figures 10 and 13. It is not clear what in the measurement process produced these residual oscillations, although the points selected would have been chosen along longitudes as the CMM moved from top to bottom of the positioned phantom. These periodicities are possible considering the North to South symmetry across the equator in the sphere model fit. Next we examined the possible affects of these residual oscillations on the sphere volume estimates.

If we compare the volume results for the coarse Green and dense Green data we get the following. From Table 3 and Table 5 there appears to be only a change of about 1\% from Green 4 volume to Dense Green 1 and similarly for Green 5 to Dense Green 2. Green 6 and Dense Green 3 volumes differ by about $0.08 \%$. Therefore the oscillations in the residuals for the Dense Green 3 data do not seem to have affected the volume estimate. The residuals themselves in Fig. 10 oscillate between $-0.15 \mathrm{~mm}$ and $0.1 \mathrm{~mm}$. However, when we compare the Pink phantom volume estimates we get a vastly different result. Whereas the residuals in Figures 11 and 12 fall within the range of -0.4 to 0.15 $\mathrm{mm}$, the residuals as shown in Fig. 13 oscillate between $-0.6 \mathrm{~mm}$ and $0.6 \mathrm{~mm}$, about six times the residual oscillations of the Dense Green 3 data. The damping of the oscillations may have been due to the slight non-spherical shape of the Pink phantom, although this is a conjecture. The volume 


\begin{tabular}{||c|c|c|c||}
\hline \hline \multicolumn{4}{|c||}{ Spherical Fit Results } \\
\hline Properties & Green 4 & Green 5 & Green 6 \\
\hline \hline Center x & $0.2150 \times 10^{-2}$ & $0.2128 \times 10^{-2}$ & $0.2579 \times 10^{-2}$ \\
\hline Center y & $-0.4694 \times 10^{-2}$ & $-0.4813 \times 10^{-2}$ & $-0.4611 \times 10^{-2}$ \\
\hline Center z & $-0.5482 \times 10^{-1}$ & $-0.5489 \times 10^{-1}$ & $-0.5442 \times 10^{-1}$ \\
\hline Radius & 10.1124 & 10.1121 & 10.1118 \\
\hline Est. Volume & 4331.7 & 4331.3 & 4330.8 \\
\hline Mean Rad. Residual & $-1.7530 \times 10^{-4}$ & $-1.764 \times 10^{-4}$ & $-1.4684 \times 10^{-4}$ \\
\hline Mean Abs. Rad. Residual & $0.4592 \times 10^{-1}$ & $0.4607 \times 10^{-1}$ & $0.4586 \times 10^{-1}$ \\
\hline Stand. Dev. Rad. Residual & $0.5521 \times 10^{-1}$ & $0.5538 \times 10^{-1}$ & $0.5518 \times 10^{-1}$ \\
\hline Stand. Dev. Abs. Rad. Residual & $0.3038 \times 10^{-1}$ & $0.3043 \times 10^{-1}$ & $0.3041 \times 10^{-1}$ \\
\hline Expanded Uncert. Center x & $0.1849 \times 10^{-3}$ & $0.9352 \times 10^{-4}$ & $0.1844 \times 10^{-3}$ \\
\hline Expanded Uncert. Center y & $0.1849 \times 10^{-3}$ & $0.9351 \times 10^{-4}$ & $0.1844 \times 10^{-3}$ \\
\hline Expanded Uncert. Center z & $0.2249 \times 10^{-3}$ & $0.1138 \times 10^{-3}$ & $0.2243 \times 10^{-3}$ \\
\hline Expanded Uncert. Radius & $0.6551 \times 10^{-4}$ & $0.3314 \times 10^{-4}$ & $0.6535 \times 10^{-4}$ \\
\hline \hline
\end{tabular}

Table 3: Results of a Spherical Fit to Coarse Data for the Green Phantom

\begin{tabular}{||c|c|c|c||}
\hline \hline \multicolumn{4}{|c||}{ Spherical Fit Results } \\
\hline Properties & Pink 5 & Pink 6 & Pink 7 \\
\hline \hline Center x & $-0.8612 \times 10^{-2}$ & $-0.8235 \times 10^{-2}$ & $-0.8373 \times 10^{-2}$ \\
\hline Center y & $0.1572 \times 10^{-1}$ & $0.1547 \times 10^{-1}$ & $0.1555 \times 10^{-1}$ \\
\hline Center z & $0.2315 \times 10^{-1}$ & $0.2365 \times 10^{-1}$ & $0.2359 \times 10^{-1}$ \\
\hline Radius & 9.7337 & 9.7334 & 9.7329 \\
\hline Est. Volume & 3863.02 & 3862.61 & 3862.03 \\
\hline Mean Rad. Residual & $-0.2222 \times 10^{-2}$ & $-0.2721 \times 10^{-2}$ & $-0.2356 \times 10^{-2}$ \\
\hline Mean Abs. Rad. Residual & 0.1748 & 0.1746 & 0.1746 \\
\hline Stand. Dev. Rad. Residual & 0.2128 & 0.2127 & 0.2126 \\
\hline Stand. Dev. Abs. Rad. Residual & 0.1203 & 0.1203 & 0.1203 \\
\hline Expanded Uncert. Center x & $0.3617 \times 10^{-1}$ & $0.3607 \times 10^{-1}$ & $0.3602 \times 10^{-1}$ \\
\hline Expanded Uncert. Center y & $0.3635 \times 10^{-1}$ & $0.3626 \times 10^{-1}$ & $0.3620 \times 10^{-1}$ \\
\hline Expanded Uncert. Center $\mathrm{z}$ & $0.4428 \times 10^{-1}$ & $0.4415 \times 10^{-1}$ & $0.4407 \times 10^{-1}$ \\
\hline Expanded Uncert. Radius & $0.1287 \times 10^{-1}$ & $0.1283 \times 10^{-1}$ & $0.1281 \times 10^{-1}$ \\
\hline \hline
\end{tabular}

Table 4: Results of a Spherical Fit to Coarse Data for the Pink Phantom 


\begin{tabular}{||c|c|c|c||}
\hline \hline \multicolumn{4}{|c||}{ Spherical Fit Results } \\
\hline Properties & Dense Green 1 & Dense Green 2 & Dense Green 3 \\
\hline Center x & $-0.1073 \times 10^{-1}$ & $0.2112 \times 10^{-1}$ & $-0.1321 \times 10^{-1}$ \\
\hline Center y & $0.5112 \times 10^{-1}$ & $0.8661 \times 10^{-2}$ & $-0.4700 \times 10^{-1}$ \\
\hline Center z & 0.2550 & $0.4723 \times 10^{-1}$ & 0.2139 \\
\hline Radius & 10.0706 & 10.0710 & 10.1146 \\
\hline Est. Volume & 4278.1 & 4278.7 & 4334.5 \\
\hline Mean Rad. Residual & $-0.9344 \times 10^{-4}$ & $-0.7116 \times 10^{-4}$ & $-0.8881 \times 10^{-4}$ \\
\hline Mean Abs. Rad. Residual & $0.3502 \times 10^{-1}$ & $0.3267 \times 10^{-1}$ & $0.3980 \times 10^{-1}$ \\
\hline Stand. Dev. Rad. Residual & $0.4566 \times 10^{-1}$ & $0.3935 \times 10^{-1}$ & $0.4904 \times 10^{-1}$ \\
\hline Stand. Dev. Abs. Rad. Residual & $0.2918 \times 10^{-1}$ & $0.2181 \times 10^{-1}$ & $0.2850 \times 10^{-1}$ \\
\hline Expanded Uncert. Center x & $0.5320 \times 10^{-4}$ & $0.2980 \times 10^{-4}$ & $0.7197 \times 10^{-4}$ \\
\hline Expanded Uncert. Center y & $0.5324 \times 10^{-4}$ & $0.2980 \times 10^{-4}$ & $0.7158 \times 10^{-4}$ \\
\hline Expanded Uncert. Center z & $0.1233 \times 10^{-3}$ & $0.7006 \times 10^{-4}$ & $0.1670 \times 10^{-3}$ \\
\hline Expanded Uncert. Radius & $0.3058 \times 10^{-4}$ & $0.1778 \times 10^{-4}$ & $0.4158 \times 10^{-4}$ \\
\hline \hline
\end{tabular}

Table 5: Results of a Spherical Fit to Dense Data for the Green Phantom

differences between Pink 5 in Table 4 and Dense Pink 1 in Table 6 as well as Pink 6 and Dense Pink 2 in the same tables are approximately $6 \%$. The volume estimates between the Pink 7 results in Table 4 and the Dense Pink 3 results in Table 6, however, show about a 7\% decrease. This last comparison may not be a fair one in that the Pink 7 data did not have data for the Pink phantom in position 3. Table 1 shows that the diameter of the Pink phantom falls approximately between 20 $\mathrm{mm}$ and $18.5 \mathrm{~mm}$. This would imply that the sphere model should have a volume between 4188.8 $\mathrm{mm}^{3}$ and $3315.2 \mathrm{~mm}^{3}$. Table 4 for the Pink phantom shows that for the coarse data the volume estimate falls from $3862 \mathrm{~mm}^{3}$ to about $3863 \mathrm{~mm}^{3}$. These volumes are within the expected range. Whereas the volume estimates for Dense Pink 1, Dense Pink 2, and Dense Pink 3 from Table 6 are also within the expected range. The Pink phantom in position 3 may have shown difficulties in the data acquisition phase for the CMM. This could explain the lower volume estimate. In particular, an approximate $4 \%$ difference in radius estimate between Dense Pink 3 and Dense Pink 2 causes an approximate $13 \%$ decrease in volume estimate. This points how seemeingly small differences in linear parameter estiamtes can make significant volumetric differences.

\subsection{Data Modeling by B-Splines}

In the following sections we plan to introduce a more general approach to estimate the phantom volumes by modeling the surfaces with B-splines and using the Divergence Theorem to estimate the volume, although in the case of the Green phantom the sphere model and the B-spline model will confirm its near spherical nature.

\subsubsection{Euclidean to Spherical Coordinate Transformation}

The current study involves the modeling of the sets of CMM data points. These unstructured sets of $(x, y, z)$ data points are called point clouds. Therefore, in this section we assume that we are given a set of $n$ points, $\left(x_{i}, y_{i}, z_{i}\right), i=1,2, \cdots, n$, for some $n$, on a surface. As measured, these points are given relative to the CMM origin. The first step is to develop an origin for the data by using the 


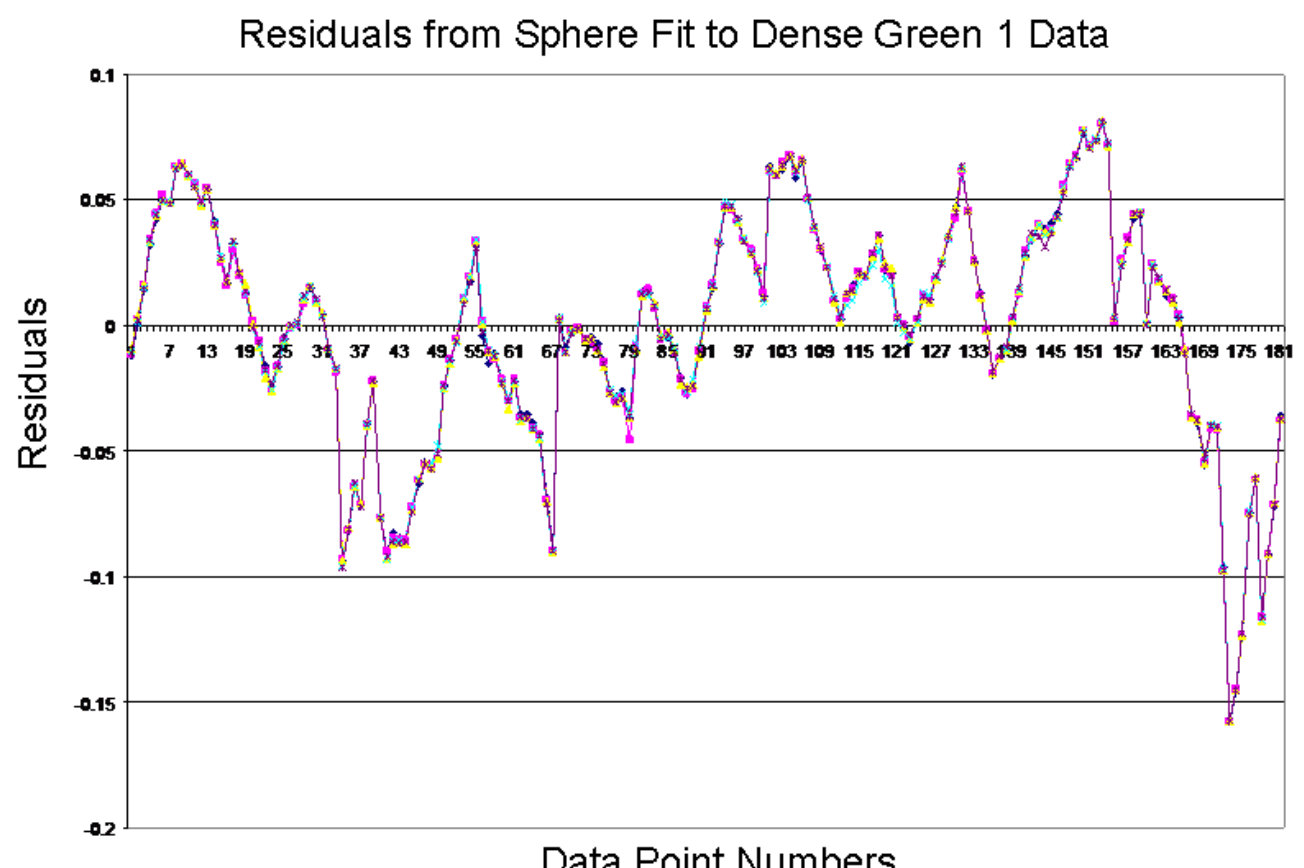

Data Point Numbers

Figure 8: Residuals for the Sphere Fit to the Dense Green Data Set 1. 


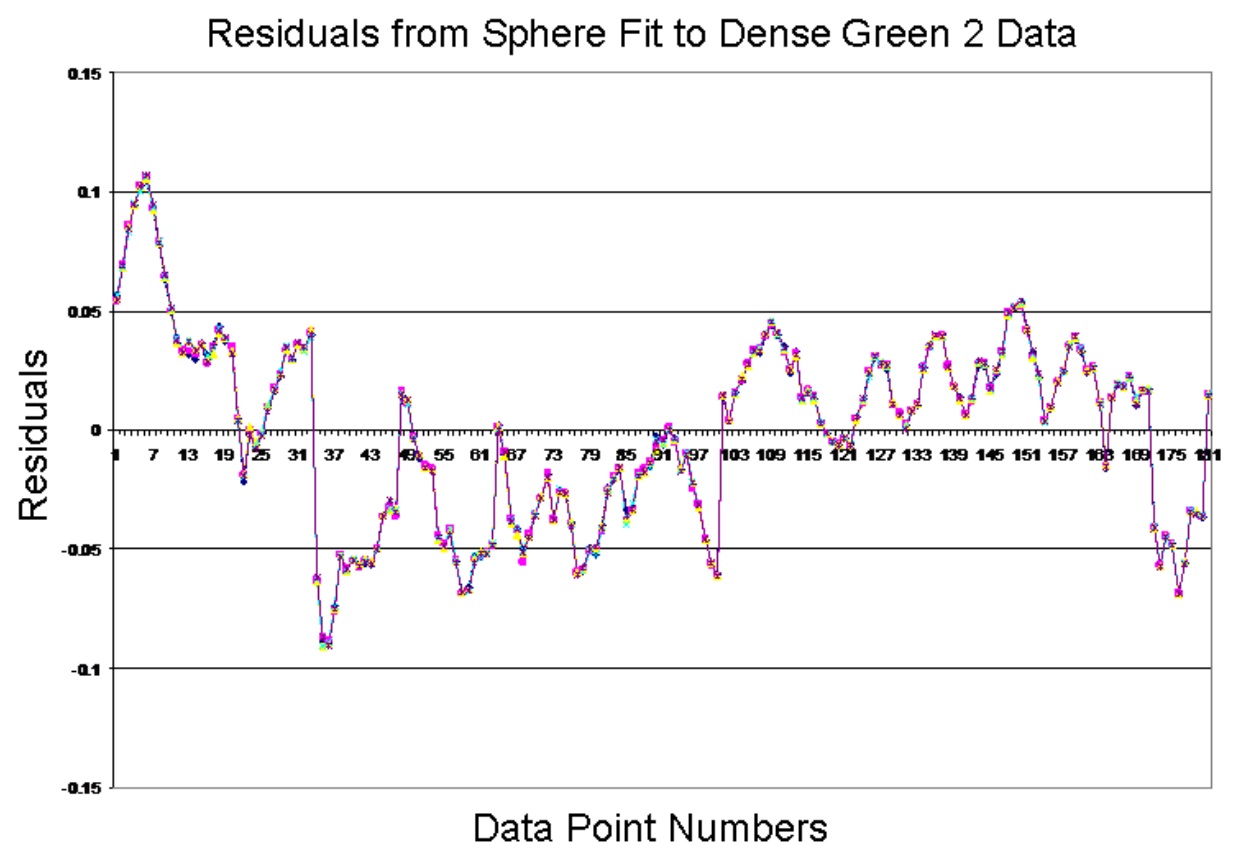

Figure 9: Residuals for the Sphere Fit to the Dense Green Data Set 2. 


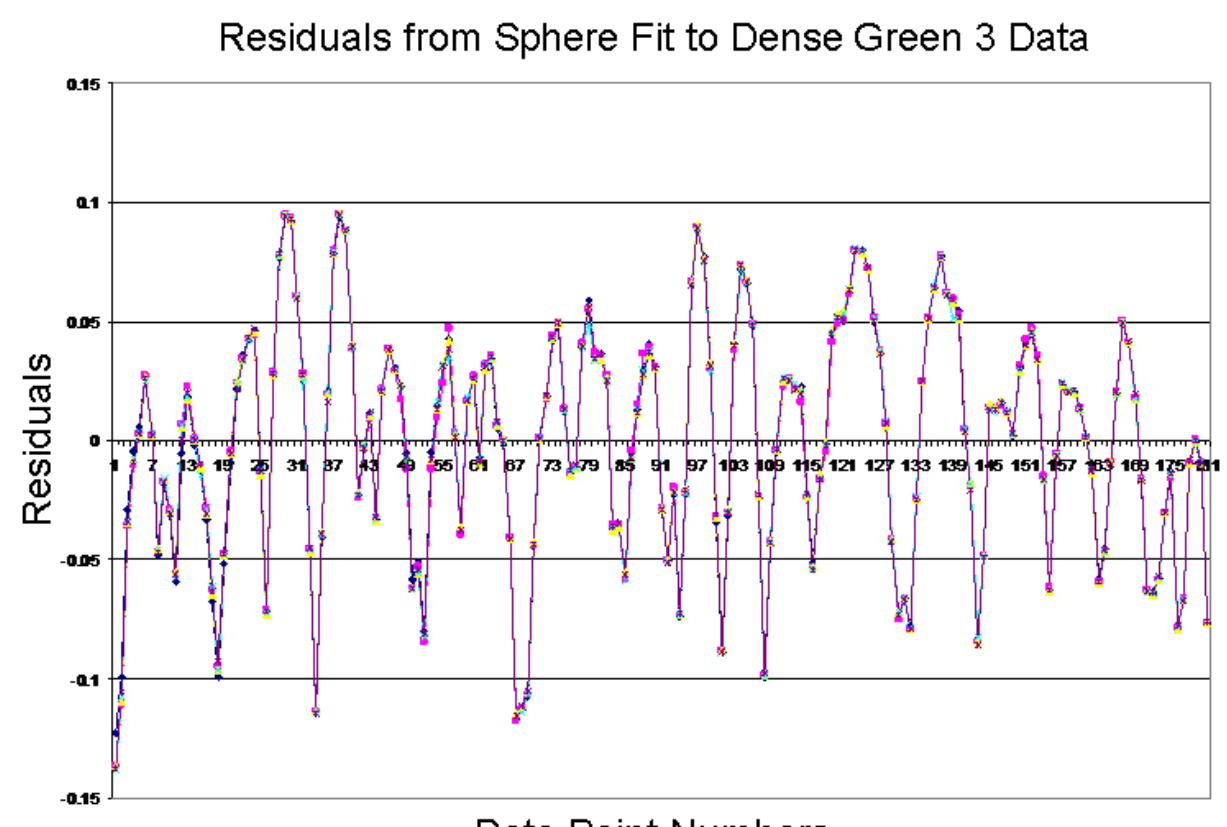

Data Point Numbers

Figure 10: Residuals for the Sphere Fit to the Dense Green Data Set 3. 


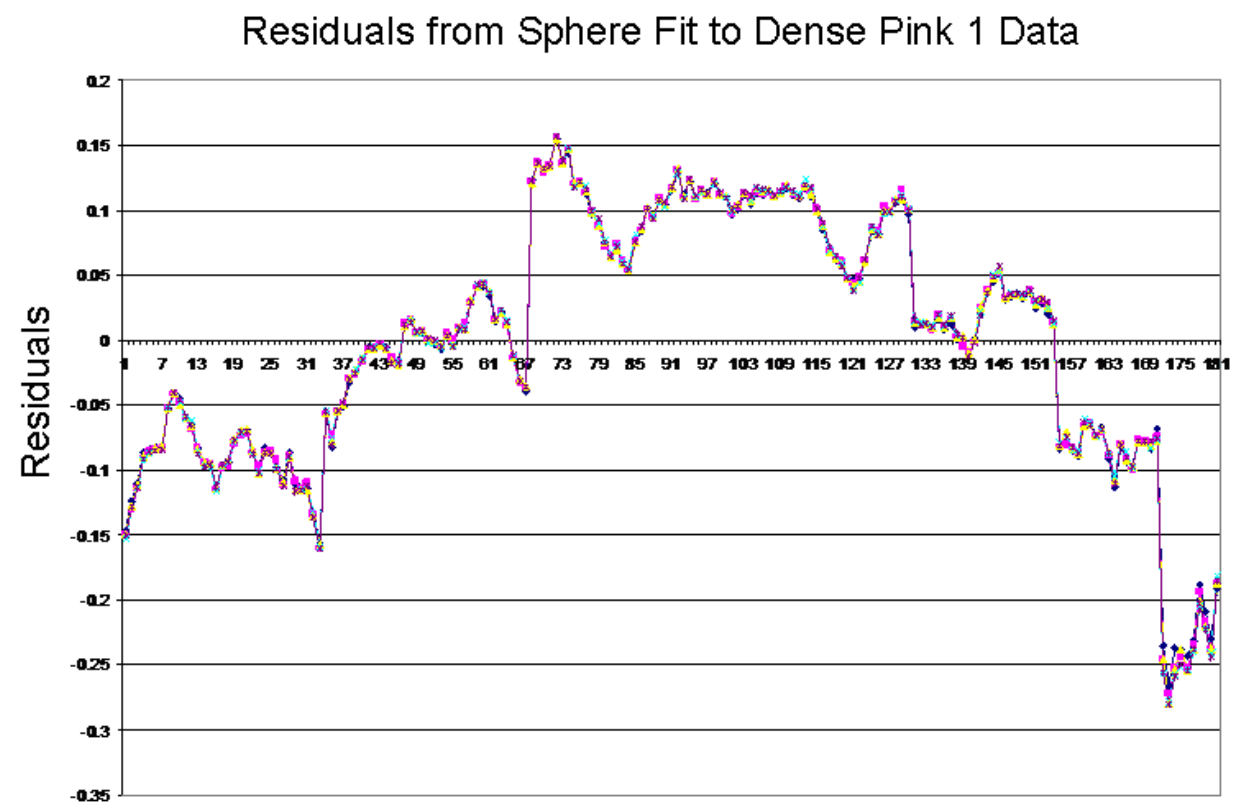

Data Point Numbers

Figure 11: Residuals for the Sphere Fit to the Dense Pink Data Set 1. 


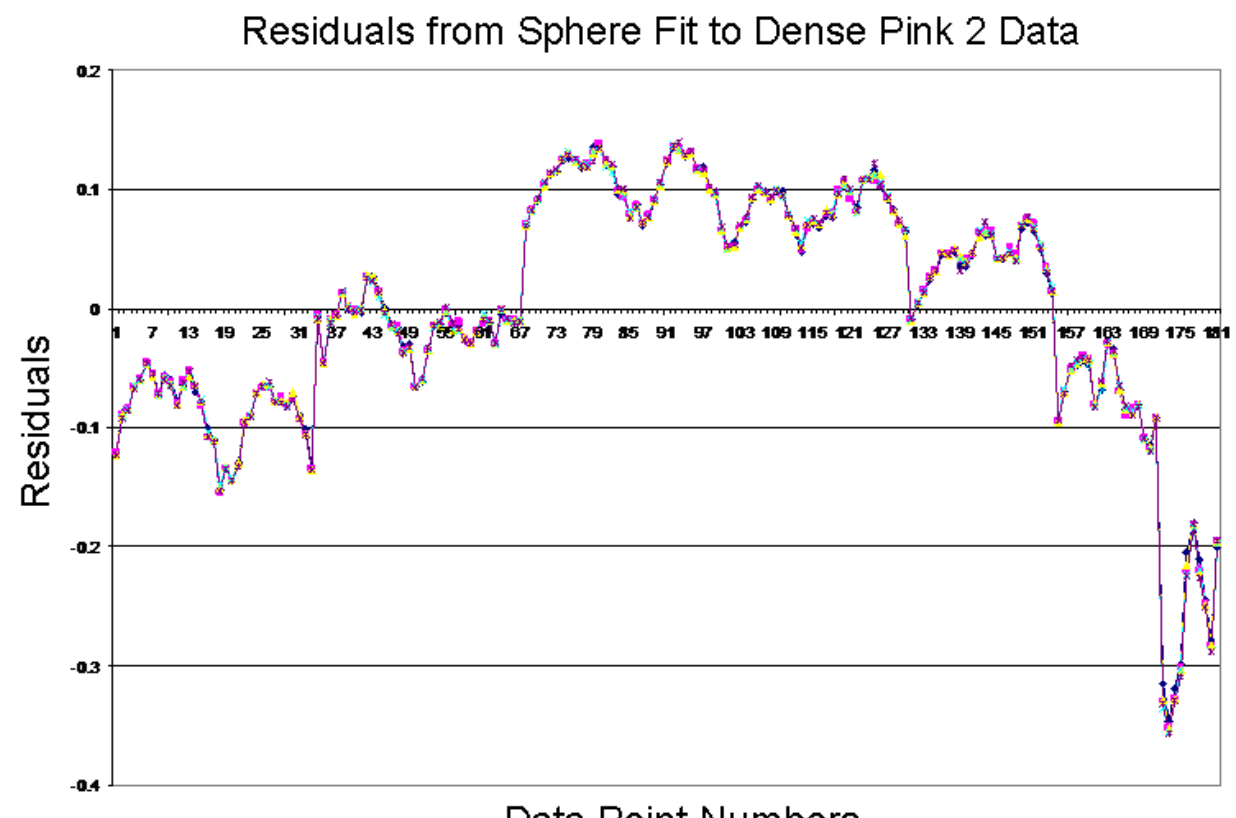

Data Point Numbers

Figure 12: Residuals for the Sphere Fit to the Dense Pink Data Set 2. 


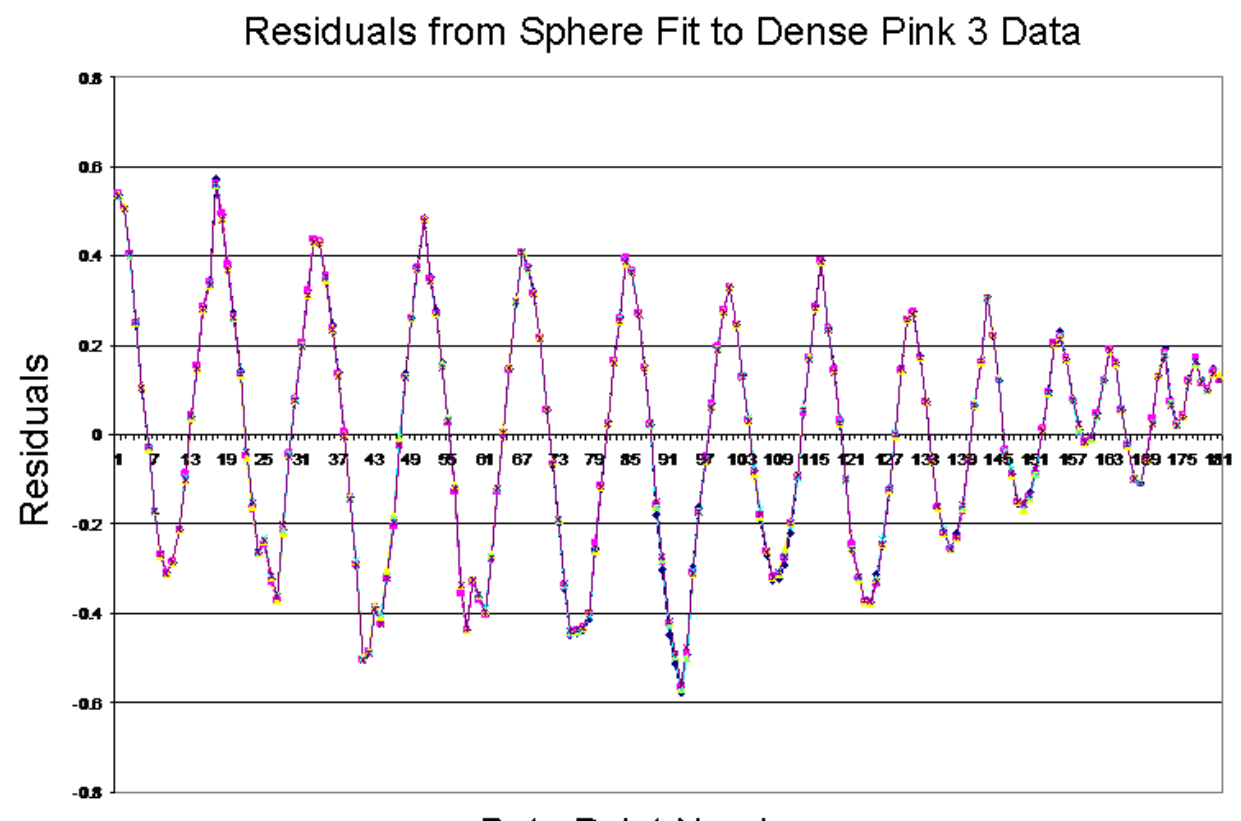

Data Point Numbers

Figure 13: Residuals for the Sphere Fit to the Dense Pink Data Set 3. 


\begin{tabular}{||c|c|c|c||}
\hline \hline \multicolumn{4}{|c||}{ Spherical Fit Results } \\
\hline Properties & Dense Pink 1 & Dense Pink 2 & Dense Pink 3 \\
\hline \hline Center x & $0.1472 \times 10^{-1}$ & $0.4573 \times 10^{-1}$ & $0.1496 \times 10^{-1}$ \\
\hline Center y & $0.1448 \times 10^{-1}$ & $0.1557 \times 10^{-1}$ & $0.6815 \times 10^{-1}$ \\
\hline Center z & 0.6848 & 0.7473 & -0.3443 \\
\hline Radius & 9.9234 & 9.9370 & 9.5045 \\
\hline Est. Volume & 4093.2 & 4110.1 & 3596.5 \\
\hline Mean Rad. Residual & $-0.4393 \times 10^{-3}$ & $-0.4859 \times 10^{-3}$ & $-0.3282 \times 10^{-2}$ \\
\hline Mean Abs. Rad. Residual & $0.7993 \times 10^{-1}$ & $0.7989 \times 10^{-1}$ & 0.2149 \\
\hline Stand. Dev. Rad. Residual & $0.9764 \times 10^{-1}$ & 0.1001 & 0.2567 \\
\hline Stand. Dev. Abs. Rad. Residual & $0.5576 \times 10^{-1}$ & $0.5996 \times 10^{-1}$ & 0.1397 \\
\hline Expanded Uncert. Center x & $0.1060 \times 10^{-2}$ & $0.1162 \times 10^{-2}$ & $0.4686 \times 10^{-1}$ \\
\hline Expanded Uncert. Center y & $0.1057 \times 10^{-2}$ & $0.1157 \times 10^{-2}$ & $0.5073 \times 10^{-1}$ \\
\hline Expanded Uncert. Center z & $0.2398 \times 10^{-2}$ & $0.2610 \times 10^{-2}$ & 0.1178 \\
\hline Expanded Uncert. Radius & $0.5662 \times 10^{-3}$ & $0.6129 \times 10^{-3}$ & $0.3137 \times 10^{-1}$ \\
\hline \hline
\end{tabular}

Table 6: Results of a Spherical Fit to Dense Data for the Pink Phantom

center-of-data mass of the point cloud. This point is given by

$$
\begin{aligned}
\bar{x} & =\frac{1}{n} \sum_{i=1}^{n} x_{i} \\
\bar{y} & =\frac{1}{n} \sum_{i=1}^{n} y_{i} \\
\bar{z} & =\frac{1}{n} \sum_{i=1}^{n} z_{i}
\end{aligned}
$$

We note here, implicitly, that we have assumed that the point cloud can be enclosed in a spherical bounded region. In order to use spherical coordinates to represent points on the boundary of a surface we need to restrict our analysis to surfaces that are called star-shaped. These are surfaces in which a ray drawn from the center-of-data mass intersects the boundary in a unique point.

To each point in the point cloud there is a vector from $(\bar{x}, \bar{y}, \bar{z})$ to $\left(x_{i}, y_{i}, z_{i}\right)$ given by $V_{i}=$ $\left(x_{i}, y_{i}, z_{i}\right)-(\bar{x}, \bar{y}, \bar{z})$. Furthermore, any point within the bounding sphere can be identified by spherical coordinates of the form $Q(\theta, \phi)=(r \sin \theta \cos \phi, r \sin \theta \sin \phi, r \cos \theta)$, where $x=r \sin \theta \cos \phi, y=$ $r \sin \theta \sin \phi, z=r \cos \theta$, for $0 \leq \theta \leq \pi, 0 \leq \phi \leq 2 \pi$. $\theta$ is referred to as the colatitude and $\phi$ is referred to as the azimuth. Table 7 associates the three dimensional octants with their spherical coordinates, where we have suppressed $r$. The conversion to spherical coordinates was necessary since tensor product B-splines need to be defined on rectangular regions. Therefore, the Euclidean coordinates were converted to $\theta, \phi$ angles on a rectangle $[0, \pi] \times[0,2 \pi]$. The height at each $\theta, \phi$ was taken as the estimated radius from the center-of-data mass of all of the Euclidean coordinates.

Points, not necessarily on a spherical surface of radius $r$, will in general be a function of $\theta$ and $\phi$. At this point we will start with an $(x, y, z)$ point from the point cloud and compute the value of $(r(\theta, \phi), \theta, \phi)$ relative to the center-of-data mass. There are eight cases to consider. In all cases we assume that $r(\theta, \phi) \geq 0$. $r$ will always be taken so that $r^{2}=x^{2}+y^{2}+z^{2}$.

Before considering individual cases there are some general relations that apply to multiple cases. For example, in cases one through four in Table 7 we have $z \geq 0$ so that $0 \leq z \leq r$ and we will 


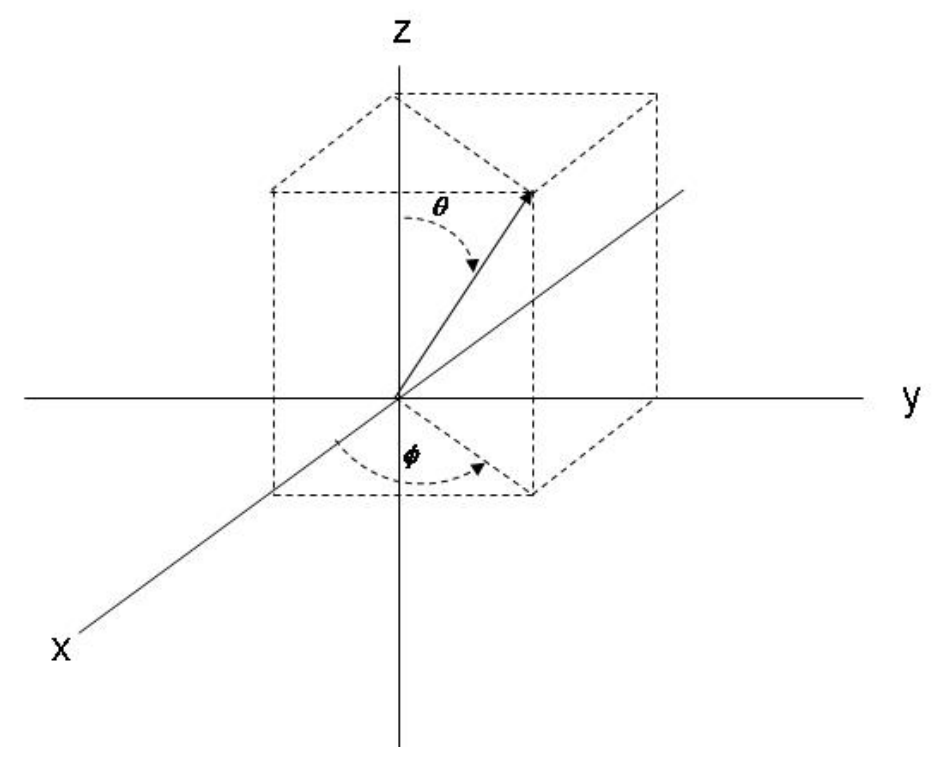

Figure 14: Spherical Coordinate angles. $\theta$ is the colatitude and $\phi$ is the azimuth.

\begin{tabular}{|c|c|c|}
\hline \multicolumn{3}{|c|}{ 3-D Octants to Spherical Coordinates } \\
\hline Octant Number & Cartesian Coordinate & Spherical Coordinate \\
\hline \hline 1 & $x \geq 0, y \geq 0, z \geq 0$ & $0 \leq \theta \leq \pi / 2,0 \leq \phi \leq \pi / 2$ \\
\hline 2 & $x<0, y \geq 0, z \geq 0$ & $0 \leq \theta \leq \pi / 2, \pi / 2 \leq \phi \leq \pi$ \\
\hline 3 & $x<0, y<0, z \geq 0$ & $0 \leq \theta \leq \pi / 2, \pi \leq \phi \leq 3 \pi / 2$ \\
\hline 4 & $x \geq 0, y<0, z \geq 0$ & $0 \leq \theta \leq \pi / 2,3 \pi / 2 \leq \phi \leq 2 \pi$ \\
\hline 5 & $x \geq 0, y \geq 0, z<0$ & $\pi / 2 \leq \theta \leq \pi / 2,0 \leq \phi \leq \pi / 2$ \\
\hline 6 & $x<0, y \geq 0, z<0$ & $\pi / 2 \leq \theta \leq \pi, \pi / 2 \leq \phi \leq \pi$ \\
\hline 7 & $x<0, y<0, z<0$ & $\pi / 2 \leq \theta \leq \pi, \pi \leq \phi \leq 3 \pi / 2$ \\
\hline 8 & $x \geq 0, y<0, z<0$ & $\pi / 2 \leq \theta \leq \pi, 3 \pi / 2 \leq \phi \leq 2 \pi$ \\
\hline
\end{tabular}

Table 7: Octant Equivalence between Euclidean and Spherical Coordinates 
always have $0 \leq z / r \leq 1$. For MATLAB this implies that

$$
0 \leq \theta=\arccos \left(\frac{z}{r}\right) \leq \frac{\pi}{2}
$$

In cases five through eight in Table $7 z<0$ so that $-1 \leq z / r<0$. Then

$$
\frac{\pi}{2} \leq \theta=\arccos \left(\frac{z}{r}\right) \leq \pi \text {. }
$$

There are also some special cases. In particular, if $x=0, y=0, z=r$, then we set $\theta=0$, and, if $x=0, y=0, z=-r$, then we set $\theta=\pi$.

In Octant $1, x \geq 0, y \geq 0, z \geq 0$. We assume $(0,0,0)$ will not be considered. We estimate $r=\sqrt{x^{2}+y^{2}+z^{2}}$. We have $\theta$ from (24), so now we want to compute the associated $\phi$. For $\theta \in(0, \pi / 2)$ we have $0<x /(r \sin \theta)<1$. Then

$$
0<\phi=\arccos \left(\frac{x}{r \sin \theta}\right)<\frac{\pi}{2} .
$$

In Octant $2, x<0, y \geq 0, z \geq 0$. Set $r=\sqrt{x^{2}+y^{2}+z^{2}}$ and $\theta$ from (24). Since $0 \leq \theta<\pi / 2$, then $-1<x /(r \sin \theta)<0$. Therefore

$$
\frac{\pi}{2}<\phi=\arccos \left(\frac{x}{r \sin \theta}\right)<\pi
$$

the desired interval.

In Octant $3, x<0, y<0, z \geq 0$. Set $r=\sqrt{x^{2}+y^{2}+z^{2}}$ and $\theta$ from (24). We want $\pi \leq \phi \leq 3 \pi / 2$. Since $-1<x /(r \sin \theta)<0$ then $\pi / 2<\phi_{1}=\arccos (x /(r \sin \theta))<\pi$ and

$$
\pi<\phi=\phi_{1}+\frac{\pi}{2}<\frac{3 \pi}{2} .
$$

In Octant $4, x \geq 0, y<0, z \geq 0$. Set $r=\sqrt{x^{2}+y^{2}+z^{2}}$ and $\theta$ from (24). We want $3 \pi / 2 \leq \phi \leq$ $2 \pi$. Since $0<x /(r \sin \theta)<1$, then $0<\phi_{1}=\arccos (x /(r \sin \theta))<\pi / 2$

$$
\frac{3 \pi}{2} \leq \phi=\phi_{1}+\frac{3 \pi}{2} \leq 2 \pi \text {. }
$$

In Octant $5, x \geq 0, y \geq 0, z<0$. Set $r=\sqrt{x^{2}+y^{2}+z^{2}}$ and solve for $\theta$ from (24). Since $0<x /(r \sin \theta)<1$,

$$
0<\phi=\arccos \left(\frac{x}{r \sin \theta}\right) \leq \frac{\pi}{2} .
$$

In Octant $6, x<0, y \geq 0, z<0$. Set $r=\sqrt{x^{2}+y^{2}+z^{2}}$ and solve for $\theta$ from (24). We want $\pi / 2 \leq \phi \leq \pi$. Since $-1<x /(r \sin \theta)<0$

$$
\frac{\pi}{2}<\phi=\arccos \left(\frac{x}{r \sin \theta}\right) \leq \pi .
$$

In Octant $7, x<0, y<0, z<0$. Set $r=\sqrt{x^{2}+y^{2}+z^{2}}$ and solve for $\theta$ from (24). We want $\pi \leq \phi \leq 3 \pi / 2$, but $\pi / 2 \leq \phi_{1}=\arccos (x /(r \sin \theta)) \leq \pi$ so we set

$$
\pi \leq \phi=\phi_{1}+\frac{\pi}{2} \leq \pi \text {. }
$$

In Octant $8, x \geq 0, y<0, z<0$. Set $r=\sqrt{x^{2}+y^{2}+z^{2}}$ and solve for $\theta$ from (24). We want $3 \pi / 2 \leq \phi \leq 2 \pi$, but $0 \leq \phi_{1}=\arccos (x /(r \sin \theta)) \leq \pi / 2$ so we set

$$
\frac{3 p i}{2}<\phi=\phi_{1}+\frac{3 \pi}{2}<2 \pi \text {. }
$$




\subsubsection{Transformation Algorithm}

The algorithm is relatively straightforward.

1. Load the measured points $\left(x_{i}, y_{i}, z_{i}\right)$ into three arrays.

2. Compute length of the arrays and set $=n$. This assumes the arrays are of equal length.

3. Compute the center of data mass

$$
\begin{aligned}
& \bar{x}=\frac{1}{n} \sum_{i=1}^{n} x_{i}, \\
& \bar{y}=\frac{1}{n} \sum_{i=1}^{n} y_{i}, \\
& \bar{z}=\frac{1}{n} \sum_{i=1}^{n} z_{i} .
\end{aligned}
$$

4. Reset the $\left(x_{i}, y_{i}, z_{i}\right)$ points as

$$
\begin{aligned}
x_{i} & =x_{i}-\bar{x} \\
y_{i} & =y_{i}-\bar{y} \\
z_{i} & =z_{i}-\bar{z}
\end{aligned}
$$

5. Begin loop from $\mathrm{i}=1$ to $\mathrm{n}$

5.1. Compute $r_{i}=\sqrt{x_{i}^{2}+y_{i}^{2}+z_{i}^{2}}$.

5.2. Test for the special cases.

5.2.1. If $x_{i}=0, y_{i}=0, z_{i}=r_{i}$, set $\theta_{i}=0, \phi_{i}=0$.

5.2.1. If $x_{i}=0, y_{i}=0, z_{i}=-r_{i}$, set $\theta_{i}=\pi, \phi_{i}=0$.

5.3. Test for the Octants

5.3.1. If $x_{i} \geq 0, y_{i} \geq 0, z_{i} \geq 0$

$$
\begin{aligned}
\theta_{i} & =\arccos \left(\frac{z_{i}}{r_{i}}\right), \\
\phi_{i} & =\arccos \left(\frac{x_{i}}{r_{i} \sin \theta_{i}}\right) .
\end{aligned}
$$

5.3.2. If $x_{i}<0, y_{i} \geq 0, z_{i} \geq 0$

$$
\begin{aligned}
\theta_{i} & =\arccos \left(\frac{z_{i}}{r_{i}}\right) \\
\phi_{i} & =\arccos \left(\frac{x_{i}}{r_{i} \sin \theta_{i}}\right) .
\end{aligned}
$$

5.3.3. If $x_{i}<0, y_{i}<0, z_{i} \geq 0$

$$
\begin{aligned}
\theta_{i} & =\arccos \left(\frac{z_{i}}{r_{i}}\right), \\
\phi_{i} & =\frac{\pi}{2}+\arccos \left(\frac{x_{i}}{r_{i} \sin \theta_{i}}\right) .
\end{aligned}
$$


5.3.4. If $x_{i} \geq 0, y_{i}<0, z_{i} \geq 0$

$$
\begin{aligned}
\theta_{i} & =\arccos \left(\frac{z_{i}}{r_{i}}\right), \\
\phi_{i} & =\frac{3 \pi}{2}+\arccos \left(\frac{x_{i}}{r_{i} \sin \theta_{i}}\right) .
\end{aligned}
$$

5.3.5. If $x_{i} \geq 0, y_{i} \geq 0, z_{i}<0$

$$
\begin{aligned}
\theta_{i} & =\arccos \left(\frac{z_{i}}{r_{i}}\right) \\
\phi_{i} & =\arccos \left(\frac{x_{i}}{r_{i} \sin \theta_{i}}\right) .
\end{aligned}
$$

5.3.6. If $x_{i}<0, y_{i} \geq 0, z_{i}<0$

$$
\begin{aligned}
\theta_{i} & =\arccos \left(\frac{z_{i}}{r_{i}}\right) \\
\phi_{i} & =\arccos \left(\frac{x_{i}}{r_{i} \sin \theta_{i}}\right) .
\end{aligned}
$$

5.3.7. If $x_{i}<0, y_{i}<0, z_{i}<0$

$$
\begin{aligned}
\theta_{i} & =\arccos \left(\frac{z_{i}}{r_{i}}\right), \\
\phi_{i} & =\frac{\pi}{2}+\arccos \left(\frac{x_{i}}{r_{i} \sin \theta_{i}}\right) .
\end{aligned}
$$

5.3.8. If $x_{i} \geq 0, y_{i}<0, z_{i}<0$

$$
\begin{aligned}
\theta_{i} & =\arccos \left(\frac{z_{i}}{r_{i}}\right) \\
\phi_{i} & =\frac{3 \pi}{2}+\arccos \left(\frac{x_{i}}{r_{i} \sin \theta_{i}}\right) .
\end{aligned}
$$

6. End loop on i.

This algorithm is implemented in the MATLAB function program in Appendix C.2. The results of the conversion of the probe points for the Green phantom from Euclidean coordinates to $\theta, \phi$ coordinates are shown in Fig. 15.

\subsection{Volume Estimation by the Divergence Theorem}

In this section we will state the Divergence Theorem and indicate how it can be used to estimate the volume of a polyhedron. 


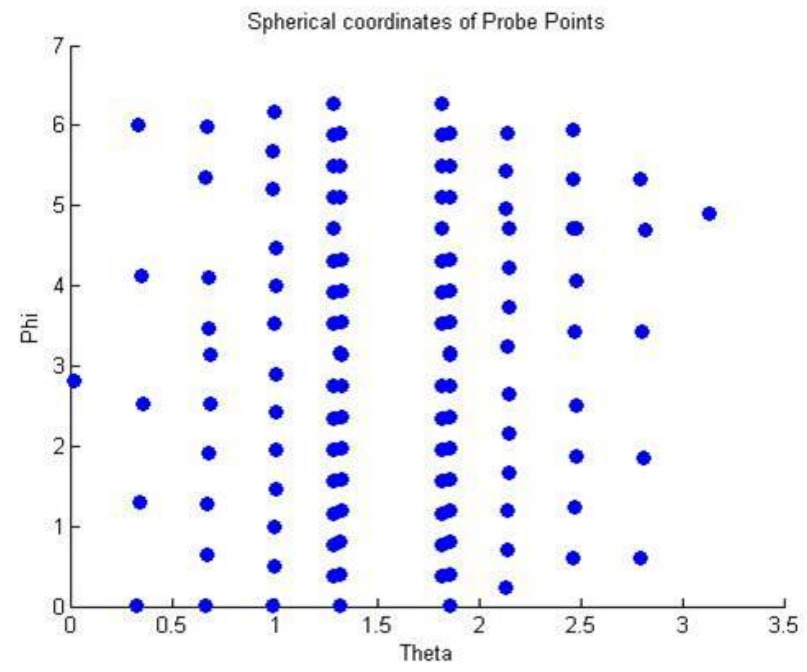

Figure 15: Plot of the $\theta, \phi$ Coordinates of the Probe Points for the Coarse Data.

\subsubsection{Divergence Theorem in 3-D and Volume Computation}

Let $R$ be a simply connected region in space and $S$ the surface boundary. Then

$$
\iint_{R} \int \vec{\nabla} \cdot F d x d y d z=\iint_{S} F \cdot \hat{n} d \sigma
$$

where $F(x, y, z)=\left(F_{1}(x, y, z), F_{2}(x, y, z), F_{3}(x, y, z)\right)$ is a differentiable vector field,

$$
\vec{\nabla} \cdot F=\frac{\partial F_{1}}{\partial x}+\frac{\partial F_{2}}{\partial y}+\frac{\partial F_{3}}{\partial z}
$$

is the divergence of the vector field, $\hat{n}$ is an outward-pointing unit normal vector to $S$, and $d \sigma$ is an infinitesimal element of the surface.

If we select $F(x, y, z)=(1 / 3)(x, y, z)$ then $\vec{\nabla} \cdot F=1$ and we can write

$$
\operatorname{Vol}(R)=\iint_{R} \int d x d y d z=\frac{1}{3} \int_{S} \int \hat{n} \cdot(x, y, z) d \sigma .
$$

Now, if $S$ is approximated by disjoint polyhedron (surface patches), then

$$
S=\bigcup_{i=1}^{N} S_{i}
$$

and

$$
\frac{1}{3} \int_{S} \int \hat{n} \cdot(x, y, z) d \sigma=\frac{1}{3} \sum_{i=1}^{N} \int_{S_{i}} \int \hat{n_{i}} \cdot(x, y, z) d \sigma,
$$

where $\hat{n}_{i}$ is the unit outer normal to $S_{i}$. Here we will model the surface patches by planar facets and, in particular, triangular facets. The plane $S_{i}$ is given by $\hat{n}_{i} \cdot(x, y, z)=c_{i}$ for a constant $c_{i}$ 
associated with each triangular facet. Since this constant applies for all points on the triangular facet, it applies for any vertex point $P_{i}$ of the triangular facet. The sum of the integrals over the facets reduces to

$$
\frac{1}{3} \sum_{i=1}^{N} \int_{S_{i}} \int \hat{n_{i}} \cdot(x, y, z) d \sigma=\frac{1}{3} \sum_{i=1}^{N} \int_{S_{i}} \int c_{i} d \sigma=\frac{1}{3} \sum_{i=1}^{N} c_{i} \operatorname{Area}\left(S_{i}\right) .
$$

This method of computing the volume of an object from a surface integral can be found in Schneider and Eberly [15].

\subsubsection{Area of a Planar Polyhedron in 3-D}

We will describe the process of computing the area of a planar polyhedron in space by use of Stokes' Theorem and then we will particularize it to a planar triangle in space. Stokes' Theorem assumes that if $C$ is a piecewise smooth boundary curve, oriented positively, of a surface $S$, and if $F$ is a differentiable vector field defined on $S$ and $\hat{n}$ is a unit normal satisfying the right-hand rule relative to the boundary orientation, then

$$
\int_{S} \int(\vec{\nabla} \times F) \cdot \hat{n} d \sigma=\int_{C} F \cdot d \vec{R}
$$

where the curl of $F(x, y, z)=\left(F_{1}(x, y, z), F_{2}(x, y, z), F_{3}(x, y, z)\right)$ is

$$
\vec{\nabla} \times F=\left(\frac{\partial F_{3}}{\partial y}-\frac{\partial F_{2}}{\partial z}, \frac{\partial F_{1}}{\partial z}-\frac{\partial F_{3}}{\partial x}, \frac{\partial F_{2}}{\partial x}-\frac{\partial F_{1}}{\partial y}\right)
$$

and $d \vec{R}=(d x, d y, d z)$. If we set $F=(1 / 2) \hat{n} \times(x, y, z)$ then $\vec{\nabla} \times F=\hat{n}$ and $\hat{n} \cdot \hat{n}=1$ so that

$$
\begin{aligned}
\operatorname{Area}(S)=\int_{S} \int d \sigma & =\frac{1}{2} \oint_{C}(\hat{n} \times(x, y, z)) \cdot(d x, d y, d z) \\
& =\frac{1}{2} \oint_{C} \hat{n} \cdot((x, y, z) \times(d x, d y, d z)) \\
& =\frac{1}{2} \oint_{C} \hat{n} \cdot\left((x(t), y(t), z(t)) \times\left(x^{\prime}(t), y^{\prime}(t), z^{\prime}(t)\right)\right) d t \\
& =\frac{1}{2} \oint_{C} \hat{n} \cdot\left(y(t) z^{\prime}(t)-z(t) y^{\prime}(t), z(t) x^{\prime}(t)-x(t) z^{\prime}(t), x(t) y^{\prime}(t)-y(t) x^{\prime}(t)\right) d t
\end{aligned}
$$

We will now specialize the Stokes formula to find the area of a spatial triangle in terms of its three vertices oriented positively. Let the three vertices, in positive orientation, be specified by $v_{1}=\left(x_{1}, y_{1}, z_{1}\right), v_{2}=\left(x_{2}, y_{2}, z_{2}\right), v_{3}=\left(x_{3}, y_{3}, z_{3}\right)$. Parameterize the boundary of the triangle as follows. On $[0,1]$ let $v=v_{1}+t\left(v_{2}-v_{3}\right)=\left(x_{1}+t\left(x_{2}-x_{1}\right), y_{1}+t\left(y_{2}-y_{1}\right), z_{1}+t\left(z_{2}-z_{1}\right)\right)$. On $[1,2]$ let $v=v_{2}+(t-1)\left(v_{3}-v_{2}\right)=\left(x_{2}+(t-1)\left(x_{3}-x_{2}\right), y_{2}+(t-1)\left(y_{3}-y_{2}\right), z_{1}+(t-1)\left(z_{3}-z_{2}\right)\right)$. Finally on $[2,3]$ let $v=v_{3}+(t-2)\left(v_{1}-v_{3}\right)=\left(x_{3}+(t-2)\left(x_{1}-x_{3}\right), y_{3}+(t-2)\left(y_{1}-y_{3}\right), z_{3}+(t-2)\left(z_{1}-z_{3}\right)\right)$. The area of the spatial triangle, in terms of the parameterized boundary, can be written as

$$
\operatorname{Area}(S)=\frac{1}{2} \oint_{C} \hat{n} \cdot\left(y(t) z^{\prime}(t)-z(t) y^{\prime}(t), z(t) x^{\prime}(t)-x(t) z^{\prime}(t), x(t) y^{\prime}(t)-y(t) x^{\prime}(t)\right) d t
$$




$$
\begin{gathered}
=\frac{1}{2} \hat{n} \cdot\left(\int_{0}^{3}\left(y(t) z^{\prime}(t)-z(t) y^{\prime}(t)\right) d t, \int_{0}^{3}\left(z(t) x^{\prime}(t)-x(t) z^{\prime}(t)\right) d t,\right. \\
\left.\int_{0}^{3}\left(x(t) y^{\prime}(t)-y(t) x^{\prime}(t)\right) d t\right) .
\end{gathered}
$$

By straightforward integration over each of the parameterized segments it is easy to show that

$$
\begin{aligned}
\operatorname{Area}(S)=\frac{1}{2} \hat{n} \cdot & \left(\left(y_{1} z_{2}-y_{2} z_{1}\right)+\left(y_{2} z_{3}-y_{3} z_{2}\right)+\left(y_{3} z_{1}-y_{1} z_{3}\right),\right. \\
& \left(z_{1} x_{2}-z_{2} x_{1}\right)+\left(z_{2} x_{3}-z_{3} x_{2}\right)+\left(z_{3} x_{1}-z_{2} x_{3}\right), \\
& \left.\left.\left(x_{1} y_{2}-x_{2} y_{1}\right)+x_{2} y_{3}-x_{3} y_{2}\right)+\left(x_{3} y_{1}-x_{1} y_{3}\right)\right)
\end{aligned}
$$

The normal vector to the oriented triangle can be computed as follows. Let $\vec{v}=\left(x_{2}-x_{1}, y_{2}-\right.$ $\left.y_{1}, z_{2}-z_{1}\right)$ and $\vec{w}=\left(x_{3}-x_{1}, y_{3}-y_{1}, z_{3}-z_{1}\right)$. Then $\vec{n}=\vec{v} \times \vec{w}=\left(\left(y_{2}-y_{1}\right)\left(z_{3}-z_{1}\right)-\left(z_{2}-\right.\right.$ $\left.\left.z_{1}\right)\left(y_{3}-y_{1}\right),\left(z_{2}-z_{1}\right)\left(x_{3}-x_{1}\right)-\left(x_{2}-x_{1}\right)\left(z_{3}-z_{1}\right),\left(x_{2}-x_{1}\right)\left(y_{3}-y_{1}\right)-\left(y_{2}-y_{1}\right)\left(x_{3}-x_{1}\right)\right)$. If we set $n_{1}=\left(y_{2}-y_{1}\right)\left(z_{3}-z_{1}\right)-\left(z_{2}-z_{1}\right)\left(y_{3}-y_{1}\right), n_{2}=\left(z_{2}-z_{1}\right)\left(x_{3}-x_{1}\right)-\left(x_{2}-x_{1}\right)\left(z_{3}-z_{1}\right), n_{3}=$ $\left(x_{2}-x_{1}\right)\left(y_{3}-y_{1}\right)-\left(y_{2}-y_{1}\right)\left(x_{3}-x_{1}\right)$ then $\|\vec{n}\|=\sqrt{n_{1}^{2}+n_{2}^{2}+n_{3}^{2}}$ and $\hat{n}=\vec{n} /\|\vec{n}\|$.

\subsection{Volume Estimation for the Phantom Grid Data}

We divided the phantom surfaces into triangular surface patches as follows. First of all we assumed that the phantom surfaces had been partitioned into $n h+1$ latitude grid points and $n v+1$ longitude grid points, where the latitude grid points were set as $\phi_{1}=0<\phi_{2}<\cdots<\phi_{n h}<\phi_{n h+1}=2 \pi$ and longitude grid points given by $\theta_{1}=0<\theta_{2}<\cdots<\theta_{n v}<\theta_{n v+1}=\pi$. At every grid point there was a radius, $r(\phi, \theta)$, calculated from the surface $\mathrm{B}$-spline model. At the north and south poles the radii was taken as the median value of the grid point radial values for the cases $\theta=0$ and $\theta=\pi$.

The patches at the north and south poles were automatically triangular patches. In fact, at the north pole, where we started, there were $n h$ triangular patches. The Euclidean points at the triangle corners for each patch were computed by

$$
\begin{aligned}
& x=r \sin (\theta) \cos (\phi) \quad 0 \leq \theta \leq \pi, \\
& y=r \sin (\theta) \sin (\phi) \quad 0 \leq \phi \leq 2 \pi, \\
& z=r \cos (\theta) .
\end{aligned}
$$

For all of the triangle facets at the north pole we designated the pole as $\left(x_{1}, y_{1}, z_{1}\right)=\left(0,0, r_{m}\right)$, where $r_{m}$ was the median value of the grid generated data for $\theta_{1}=0$. We looped through the grid points at $\theta_{2}$ and $\phi_{1}, \phi_{2}, \cdots, \phi_{n h+1}=\phi_{1}$. At each of these angle pairs there was a value $r_{k}=r\left(\phi_{k}, \theta_{2}\right), k=1,2, \cdots, n h+1$. We generated the volume from the Green's Theorem result by adding up the contributions of each facet to the volume total. We did this by initializing a variable for the volume, called vol. We then started to generate the contributions from the first layer of facets at the north pole. We set, as noted above, the north pole as $\left(x_{1}, y_{1}, z_{1}\right)$ and then set

$$
\begin{aligned}
& x_{2}=r\left(\phi_{1}, \theta_{2}\right) \sin \left(\theta_{2}\right) \cos \left(\phi_{1}\right), \\
& y_{2}=r\left(\phi_{1}, \theta_{2}\right) \sin \left(\theta_{2}\right) \sin \left(\phi_{1}\right), \\
& z_{2}=r\left(\phi_{1}, \theta_{2}\right) \cos \left(\theta_{2}\right),
\end{aligned}
$$

and

$$
\begin{aligned}
& x_{3}=r\left(\phi_{2}, \theta_{2}\right) \sin \left(\theta_{2}\right) \cos \left(\phi_{2}\right), \\
& y_{3}=r\left(\phi_{2}, \theta_{2}\right) \sin \left(\theta_{2}\right) \sin \left(\phi_{2}\right), \\
& z_{3}=r\left(\phi_{2}, \theta_{2}\right) \cos \left(\theta_{2}\right)
\end{aligned}
$$


These were set in a positive orientation. Next we computed the outward normal to the triangle facet, formed the vectors $v_{1}=\left(x_{2}, y_{2}, z_{2}\right)-\left(x_{1}, y_{1}, z_{1}\right), v_{2}=\left(x_{3}, y_{3}, z_{3}\right)-\left(x_{1}, y_{1}, z_{1}\right)$, and then formed the normalized cross product

$$
\hat{n}=\frac{v_{1} \times v_{2}}{\left\|v_{1} \times v_{2}\right\|}
$$

We then computed the contribution that this facet made to the volume as

$\operatorname{vol}=\operatorname{vol}+$

$$
\left(\hat{n} \cdot\left(x_{1}, y_{1}, z_{1}\right)\right)\left\{\hat{n} \cdot\left(\sum_{j=1}^{2}\left(y_{j} z_{j+1}-y_{j+1} z_{j}\right),\left(z_{j} x_{j+1}-z_{j+1} x_{j}\right),\left(x_{j} y_{j+1}-x_{j+1} y_{j}\right)\right)\right\} .
$$

We proceeded to the next facet in the first layer. Again $\left(x_{1}, y_{1}, z_{1}\right)$ was the north pole and we then used the previous computation to get $x_{2}=x_{3}, y_{2}=y_{3}, z_{2}=z_{3}$ and set

$$
\begin{aligned}
& x_{3}=r\left(\phi_{3}, \theta_{2}\right) \sin \left(\theta_{2}\right) \cos \left(\phi_{3}\right), \\
& y_{3}=r\left(\phi_{3}, \theta_{2}\right) \sin \left(\theta_{2}\right) \sin \left(\phi_{3}\right), \\
& z_{3}=r\left(\phi_{3}, \theta_{2}\right) \cos \left(\theta_{2}\right) .
\end{aligned}
$$

We computed the normalized cross product as for the first facet and then computed the contribution of the second facet to the volume using equation (57). We continued this process for $\theta_{2}, \phi_{j}, j=$ $1, \cdots, n h$.

We next computed the contributions of the next layers in a two step process. We looped through the $\theta$ layers from 2 to $n v-1$. The last layer at the south pole was handled separately. At each layer there were $n h$ four-vertex patches. Each patch was divided into two triangles. The four vertices of the patches were identified counterclockwise as $\left(\theta_{i}, \phi_{j}, r(i, j)\right),\left(\theta_{i+1}, \phi_{j}, r(i+1, j)\right)$, $\left(\theta_{i+1}, \phi_{j+1}, r(i+1, j+1)\right),\left(\theta_{i}, \phi_{j+1}, r(i, j+1)\right)$. For the first triangle in the patch we set

$$
\begin{aligned}
x_{1} & =r(i, j) \sin \left(\theta_{i}\right) \cos \left(\phi_{j}\right), \\
y_{1} & =r(i, j) \sin \left(\theta_{i}\right) \sin \left(\phi_{j}\right), \\
z_{1} & =r(i, j) \cos \left(\theta_{i}\right) . \\
x_{2} & =r(i+1, j) \sin \left(\theta_{i+1}\right) \cos \left(\phi_{j}\right), \\
y_{2} & =r(i+1, j) \sin \left(\theta_{i+1}\right) \sin \left(\phi_{j}\right), \\
z_{2} & =r(i+1, j) \cos \left(\theta_{i+1}\right) . \\
x_{3}= & r(i+1, j+1) \sin \left(\theta_{i+1}\right) \cos \left(\phi_{j+1}\right), \\
y_{3}= & r(i+1, j+1) \sin \left(\theta_{i+1}\right) \sin \left(\phi_{j+1}\right), \\
z_{3}= & r(i+1, j+1) \cos \left(\theta_{i+1}\right) .
\end{aligned}
$$

We then proceeded as above to compute the contribution of this triangle to the volume. For the second triangle of the patch we maintained the same $x_{1}, y_{1}, z_{1}$ and set $x_{2}=x_{3}, y_{2}=y_{3}, z_{2}=z_{3}$ and then set

$$
\begin{aligned}
& x_{3}=r(i, j+1) \sin \left(\theta_{i}\right) \cos \left(\phi_{j+1}\right), \\
& y_{3}=r(i, j+1) \sin \left(\theta_{i}\right) \sin \left(\phi_{j+1}\right), \\
& z_{3}=r(i, j+1) \cos \left(\theta_{i}\right) .
\end{aligned}
$$


Again, the volume increment due to this triangle was computed as it was above.

Finally, at the south pole there were $n h$ triangles to compute. Their vertices were chosen as follows.

$$
\begin{aligned}
x_{1}= & r(n v, j) \sin \left(\theta_{n} v\right) \cos \left(\phi_{j}\right), \\
y_{1}= & r(n v, j) \sin \left(\theta_{n} v\right) \sin \left(\phi_{j}\right), \\
z_{1}= & r(n v, j) \cos \left(\theta_{n} v\right) . \\
& x_{2}=0, \\
& y_{2}=0, \\
& z_{2}=-r(n v+1,1) . \\
x_{3}= & r(n v, j+1) \sin \left(\theta_{n v}\right) \cos \left(\phi_{j+1}\right), \\
y_{3}= & r(n v, j+1) \sin \left(\theta_{n v}\right) \sin \left(\phi_{j+1}\right), \\
z_{3}= & r(n v, j+1) \cos \left(\theta_{n v}\right) .
\end{aligned}
$$

The contribution of these triangles to the volume was computed as above. After all of the triangle contributions to the volume were computed the final volume was then computed as vol $=(1 / 6) \mathrm{vol}$.

\subsection{Computational Results for the B-spline Model}

In this section we will describe the method used to estimate volume uncertainties and discuss the results of using a B-spline surface model and the Divergence Theorem to estimate the phantom volumes. A nonparametric method to estimate the volume uncertainties was chosen since there did not exist any "ground truth" values for the Green and Pink FDA phantom volumes.

\subsubsection{Estimating Volume Uncertainties}

The nonparametric "bootstrap" method is a computer intensive technique for estimating uncertainties. It involves repeated Monte Carlo resampling from the original spherical coordinates with radii values modified by the fitting residuals, refitting the model to estimate new volumes, and finally computing an uncertainty for the process from the set of computed volumes. For a full discussion of the bootstrap see Efron and Tibshirani [9]. We will give a brief description of the bootstrp method in Section 4.6.2 in the discussion of the coarse data results, but will use the same method for estimating the volume uncertainties in the case of the dense data.

\subsubsection{Volume Estimates and Uncertainties for the Coarse Data}

In this section we state the results of computing the volumes for the two phantoms using the six coarse data sets. Sixteen surface grid cases were used and the results are shown in Tables 8 through 13. Figures 16 through 26 show the dramatic asymptotic trend of the volume estimates as the grid sizes increase. It seems clear that the volume of the Green phantom may lie somewhere in the range of $4337 \mathrm{~mm}^{3}$ and $4340 \mathrm{~mm}^{3}$ while the volume of the Pink phantom volume seems to lie somewhere in the range $3910 \mathrm{~mm}^{3}$ and $3913 \mathrm{~mm}^{3}$. In terms of percentages these volume differences are in the range of about $0.07 \%$. Figures 16 to 20 show the clear increase of the volume estimate to a stable value as the surface grid became finer. The volume estimates for the Green phantom differ from the volume estimates for the sphere fit in Table 3 by approximately $0.1 \%$ to $0.2 \%$. This is indicative 
of the fact that the Green phantom is near spherical. The fit to the Pink phantom in Tables 11 to 13 are all consistent and indicate the Pink phantom likely has a larger volume than that indicated by the sphere fit in Table 4 . The differences of the stabilized Pink volume estimates differ from the spherical fit volume estimates by approximately $2 \%$. Again this likely indicates the non-spherical nature of the Pink phantom.

The object of the uncertainty estimation procedure (bootstrap) was to develop volume uncertainty as a function of grid size. The process began with the conversion of the Euclidean data points to spherical coordinates. An automatic selection of knots was done. These steps were done once for a given data set. It was assumed that the modifications of the data made during the bootstrap would be small and not affect the knot selection. During the first pass of the bootstrap algorithm the radii of the spherical coordinates were fit by a tensor product of B-splines. The predicted values and residuals from this initial fit were called the master predicted values and master residuals respectively. The computed volume was then put in a list of volumes that would be added to in subsequent passes of the algorithm. The algorithm then iterated two hundred times as follows. In the first iteration the master residuals were sampled randomly uniformly with replacement. The resampled residuals were then added to the master predicted radii and a new fit was performed. The new computed volume was added to the volume list and the master residuals sampled randomly uniformly with replacement for the next iteration. The process continued for all two hundred iterations. The standard deviation of the volumes in the volume list was then used as an estimate of the volume uncertainty and the average volume was taken as the reference volume for the chosen grid size.

From Tables 8 to 10 the uncertainties range from $7.4 \mathrm{~mm}^{3}$ on the coarse surface grid to 3.3 $\mathrm{mm}^{3}$ on the finer grid for the Green phantom. These uncertainty values represent an approximate range of $0.2 \%$ to $0.07 \%$ of the estimated Green phantom volumes from the tables. Figures 17 to 21 graphically show the rapid descent to stable values for the uncertainties. Tables 11 to 13 show uncertainties for the Pink phantom that are about twice those for the Green phantom. This is not surprising, considering the non-spherical shape of the Pink phantom. The uncertainties for the Pink phantom have an approximate range of $0.5 \%$ to $0.2 \%$ of the estimated volumes. Figures 23 to 27 show a similar descent of the uncertainties as a function of grid refinement. In a similar manner to the volumetric behavior of the Green phantom as the grid refinement increases Figures 22 to 26 show the rapid increase to a stable value for the Pink phantom volume estimates. 


\begin{tabular}{||c|c|c|c|c||}
\hline \hline \multicolumn{5}{||c||}{ Green 4 Data Set } \\
\hline \multicolumn{5}{||c|}{ Volumes and Expanded Uncertainties vs Grid Size } \\
\hline Grid Case & $\theta$ & $\phi$ & Volume $\left(\mathrm{mm}^{3}\right)$ & Uncertainty $\left(\mathrm{mm}^{3}\right)$ \\
\hline \hline 1 & 10 & 20 & 4071.5 & 7.1 \\
\hline 2 & 20 & 40 & 4278.6 & 5.1 \\
\hline 3 & 30 & 60 & 4313.6 & 4.0 \\
\hline 4 & 40 & 80 & 4325.5 & 4.0 \\
\hline 5 & 50 & 100 & 4331.2 & 3.7 \\
\hline 6 & 60 & 120 & 4334.4 & 3.6 \\
\hline 7 & 70 & 140 & 4336.1 & 3.6 \\
\hline 8 & 80 & 160 & 4337.5 & 3.7 \\
\hline 9 & 90 & 180 & 4337.9 & 3.7 \\
\hline 10 & 100 & 200 & 4338.8 & 3.7 \\
\hline 11 & 110 & 220 & 4339.1 & 3.2 \\
\hline 12 & 120 & 240 & 4339.4 & 3.7 \\
\hline 13 & 130 & 260 & 4339.6 & 3.8 \\
\hline 14 & 140 & 280 & 4339.9 & 3.9 \\
\hline 15 & 150 & 300 & 4339.9 & 3.5 \\
\hline 16 & 160 & 320 & 4340.0 & 3.3 \\
\hline \hline
\end{tabular}

Table 8: Estimated Volumes and Uncertainties for Green 4 Data

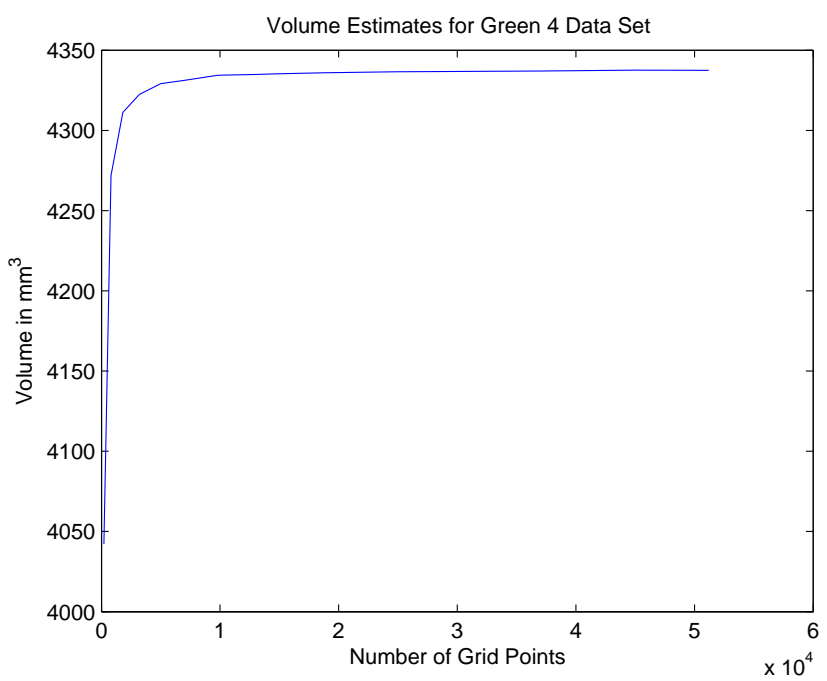

Figure 16: Volume Estimates for Green 4 Data Set vs. Grid Size. 


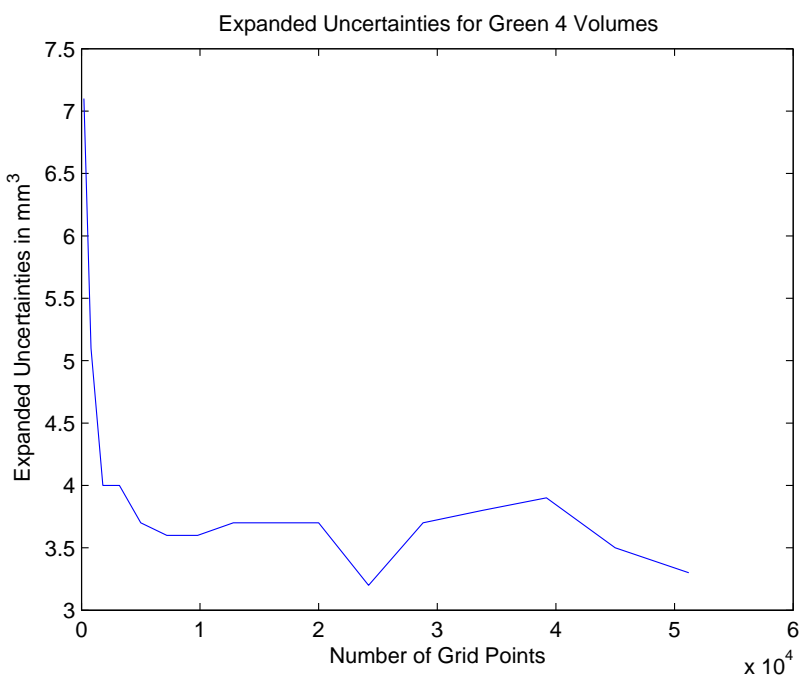

Figure 17: Uncertainty Estimates for Green 4 Data Set vs. Grid Size.

\begin{tabular}{||c|c|c|c|c||}
\hline \hline \multicolumn{5}{||c||}{ Green 5 Data Set } \\
\hline Grid Case & $\theta$ & $\phi$ & Volume $\left(\mathrm{mm}^{3}\right)$ & Uncertainty $\left(\mathrm{mm}^{3}\right)$ \\
\hline \hline 1 & 10 & 20 & 4068.5 & 7.4 \\
\hline 2 & 20 & 40 & 4275.4 & 4.8 \\
\hline 3 & 30 & 60 & 4311.4 & 4.2 \\
\hline 4 & 40 & 80 & 4323.1 & 3.8 \\
\hline 5 & 50 & 100 & 4328.5 & 3.7 \\
\hline 6 & 60 & 120 & 4331.1 & 3.7 \\
\hline 7 & 70 & 140 & 4333.2 & 3.5 \\
\hline 8 & 80 & 160 & 4334.5 & 3.6 \\
\hline 9 & 90 & 180 & 4335.1 & 3.4 \\
\hline 10 & 100 & 200 & 4335.6 & 3.4 \\
\hline 11 & 110 & 220 & 4336.1 & 3.6 \\
\hline 12 & 120 & 240 & 4336.5 & 3.5 \\
\hline 13 & 130 & 260 & 4336.8 & 3.1 \\
\hline 14 & 140 & 280 & 4336.9 & 3.3 \\
\hline 15 & 150 & 300 & 4337.0 & 3.4 \\
\hline 16 & 160 & 320 & 4337.1 & 3.5 \\
\hline \hline
\end{tabular}

Table 9: Estimated Volumes and Uncertainties for Green 5 Data 


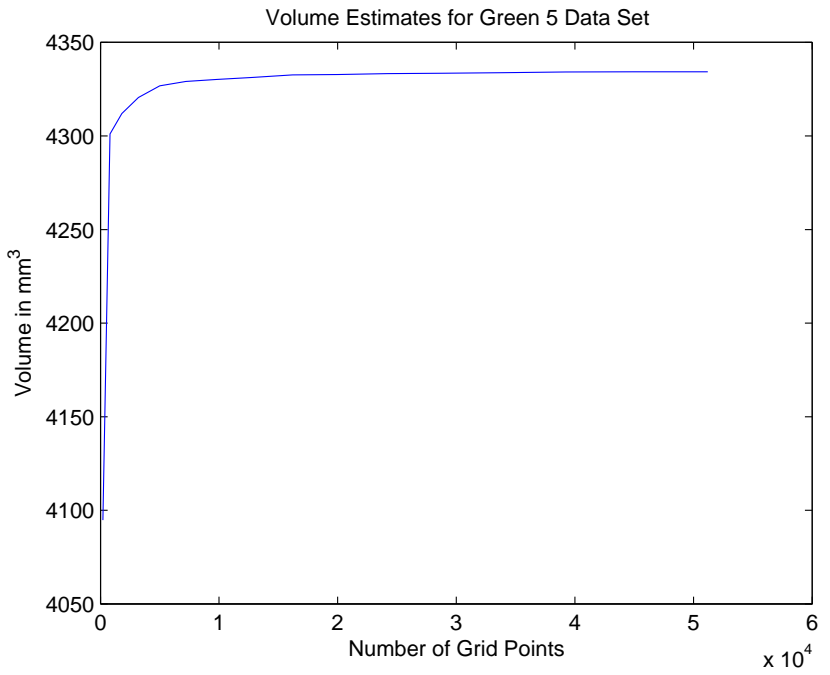

Figure 18: Volume Estimates for Green 5 Data Set vs. Grid Size.

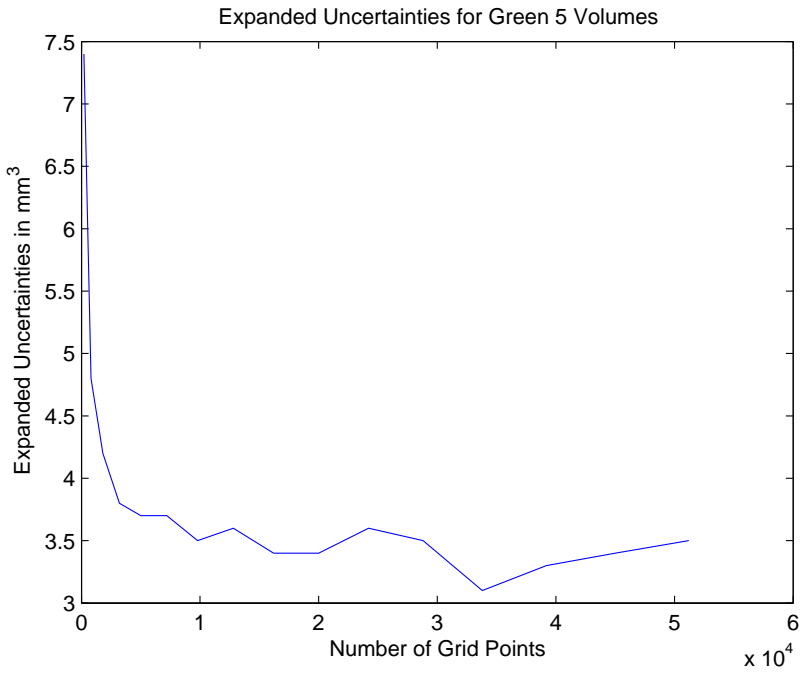

Figure 19: Uncertainty Estimates for Green 5 Data Set vs. Grid Size. 


\begin{tabular}{||c|c|c|c|c||}
\hline \hline \multicolumn{5}{|c||}{ Green 6 Data Set } \\
\hline \multicolumn{5}{||c|}{ Volumes and Expanded Uncertainties vs Grid Size } \\
\hline Grid Case & $\theta$ & $\phi$ & Volume $\left(\mathrm{mm}^{3}\right)$ & Uncertainty $\left(\mathrm{mm}^{3}\right)$ \\
\hline \hline 1 & 10 & 20 & 4071.3 & 7.4 \\
\hline 2 & 20 & 40 & 4277.6 & 4.8 \\
\hline 3 & 30 & 60 & 4312.9 & 3.8 \\
\hline 4 & 40 & 80 & 4325.0 & 4.1 \\
\hline 5 & 50 & 100 & 4330.6 & 3.4 \\
\hline 6 & 60 & 120 & 4333.7 & 3.3 \\
\hline 7 & 70 & 140 & 4335.6 & 3.4 \\
\hline 8 & 80 & 160 & 4336.9 & 3.4 \\
\hline 9 & 90 & 180 & 4337.4 & 3.9 \\
\hline 10 & 100 & 200 & 4338.0 & 3.4 \\
\hline 11 & 110 & 220 & 4338.5 & 3.8 \\
\hline 12 & 120 & 240 & 4338.9 & 3.6 \\
\hline 13 & 130 & 260 & 4339.1 & 3.4 \\
\hline 14 & 140 & 280 & 4339.6 & 3.5 \\
\hline 15 & 150 & 300 & 4339.5 & 3.4 \\
\hline 16 & 160 & 320 & 4339.8 & 3.4 \\
\hline \hline
\end{tabular}

Table 10: Estimated Volumes and Uncertainties for Green 6 Data

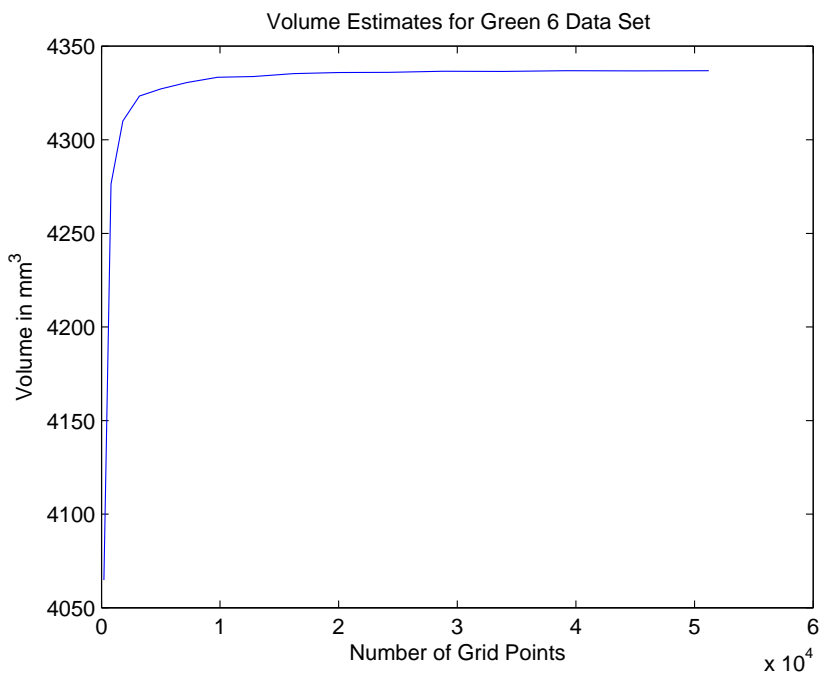

Figure 20: Volume Estimates for Green 6 Data Set vs. Grid Size. 


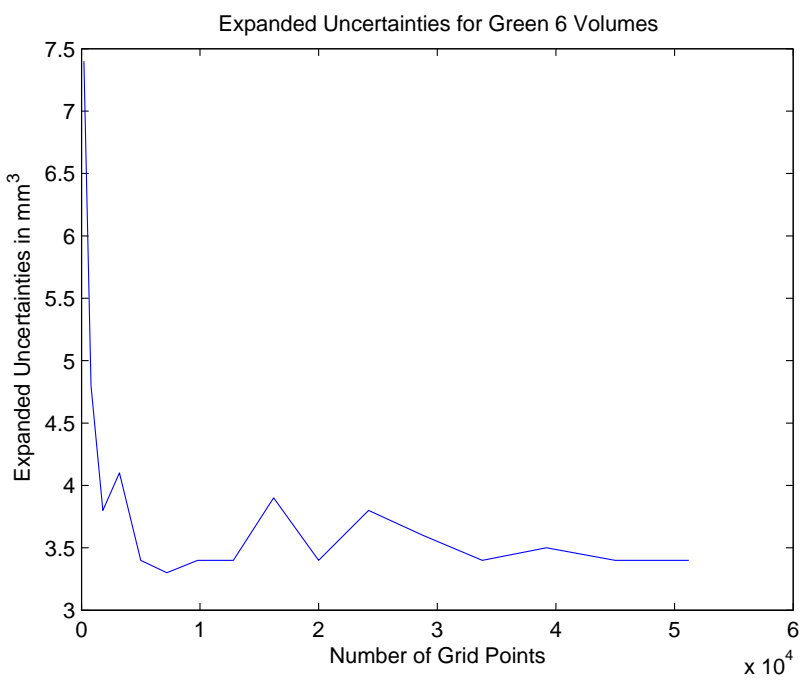

Figure 21: Uncertainty Estimates for Green 6 Data Set vs. Grid Size.

\begin{tabular}{||c|c|c|c|c||}
\hline \hline \multicolumn{6}{||c||}{ Pink 5 Data Set } \\
\hline \multicolumn{5}{||c|}{ Volumes and Expanded Uncertainties vs Grid Size } \\
\hline \hline Grid Case & $\theta$ & $\phi$ & Volume $\left(\mathrm{mm}^{3}\right)$ & Uncertainty $\left(\mathrm{mm}^{3}\right)$ \\
\hline 1 & 10 & 20 & 3697.8 & 18.7 \\
\hline 2 & 20 & 40 & 3866.1 & 9.8 \\
\hline 3 & 30 & 60 & 3899.2 & 8.7 \\
\hline 4 & 40 & 80 & 3908.8 & 7.4 \\
\hline 5 & 50 & 100 & 3914.9 & 7.4 \\
\hline 6 & 60 & 120 & 3917.1 & 6.8 \\
\hline 7 & 70 & 140 & 3919.4 & 6.9 \\
\hline 8 & 80 & 160 & 3920.7 & 6.3 \\
\hline 9 & 90 & 180 & 3921.2 & 6.8 \\
\hline 10 & 100 & 200 & 3921.8 & 6.2 \\
\hline 11 & 110 & 220 & 3922.3 & 6.7 \\
\hline 12 & 120 & 240 & 3922.6 & 7.0 \\
\hline 13 & 130 & 260 & 3922.8 & 6.7 \\
\hline 14 & 140 & 280 & 3922.9 & 6.9 \\
\hline 15 & 150 & 300 & 3922.9 & 5.8 \\
\hline 16 & 160 & 320 & 3923.2 & 6.3 \\
\hline \hline
\end{tabular}

Table 11: Estimated Volumes and Uncertainties for Pink 5 Data 


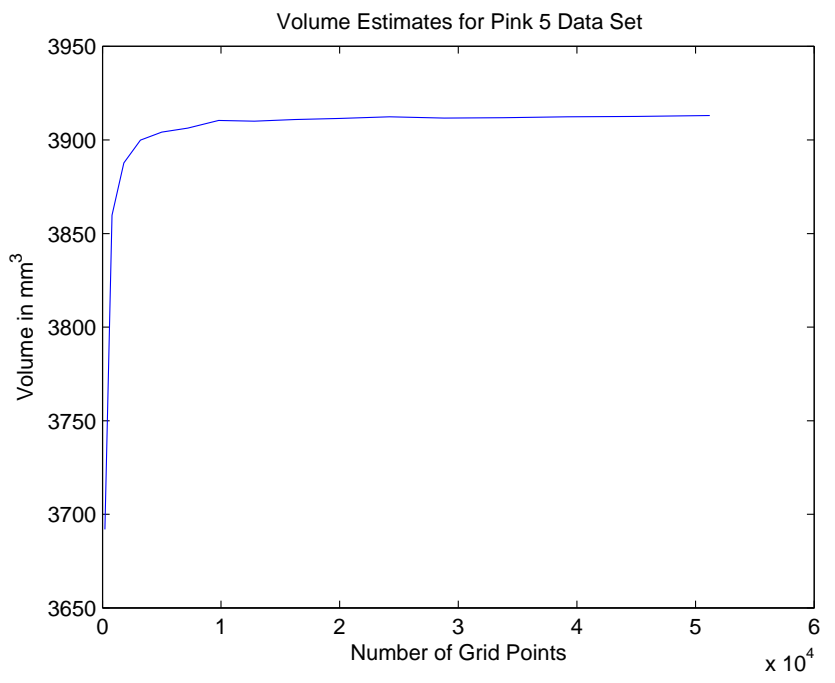

Figure 22: Volume Estimates for Pink 5 Data Set vs. Grid Size.

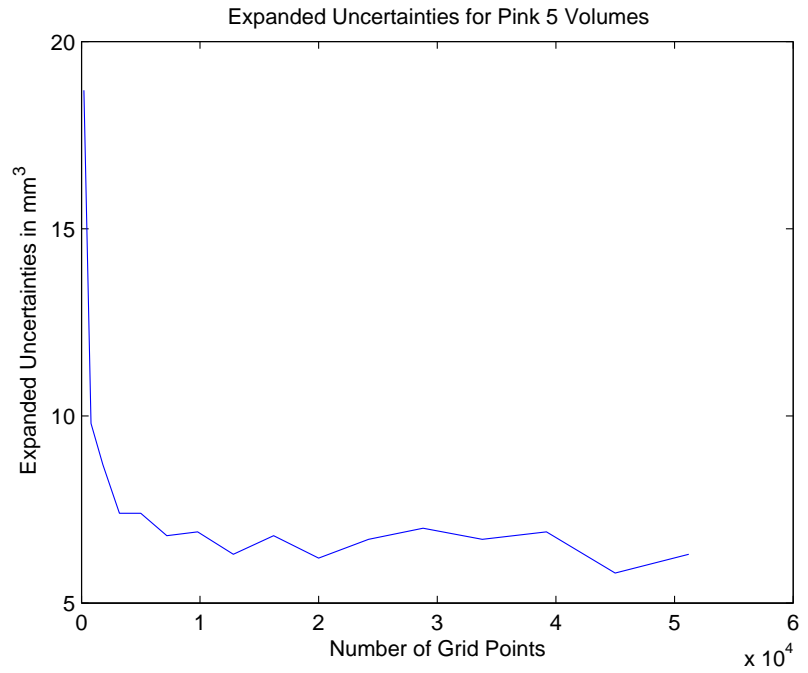

Figure 23: Uncertainty Estimates for Pink 5 Data Set vs. Grid Size. 


\begin{tabular}{||c|c|c|c|c||}
\hline \hline \multicolumn{6}{|c||}{ Pink 6 Data Set } \\
\hline \multicolumn{5}{||c|}{ Volumes and Expanded Uncertainties vs Grid Size } \\
\hline Grid Case & $\theta$ & $\phi$ & Volume $\left(\mathrm{mm}^{3}\right)$ & Uncertainty $\left(\mathrm{mm}^{3}\right)$ \\
\hline \hline 1 & 10 & 20 & 3698.3 & 17.8 \\
\hline 2 & 20 & 40 & 3864.3 & 11.2 \\
\hline 3 & 30 & 60 & 3898.2 & 8.0 \\
\hline 4 & 40 & 80 & 3907.5 & 7.7 \\
\hline 5 & 50 & 100 & 3913.5 & 7.2 \\
\hline 6 & 60 & 120 & 3916.2 & 7.0 \\
\hline 7 & 70 & 140 & 3917.6 & 6.5 \\
\hline 8 & 80 & 160 & 3919.3 & 6.3 \\
\hline 9 & 90 & 180 & 3919.8 & 6.6 \\
\hline 10 & 100 & 200 & 3920.8 & 6.8 \\
\hline 11 & 110 & 220 & 3921.0 & 6.3 \\
\hline 12 & 120 & 240 & 3921.6 & 6.4 \\
\hline 13 & 130 & 260 & 3921.2 & 6.3 \\
\hline 14 & 140 & 280 & 3922.0 & 5.9 \\
\hline 15 & 150 & 300 & 3921.4 & 5.8 \\
\hline 16 & 160 & 320 & 3922.1 & 6.6 \\
\hline \hline
\end{tabular}

Table 12: Estimated Volumes and Uncertainties for Pink 6 Data

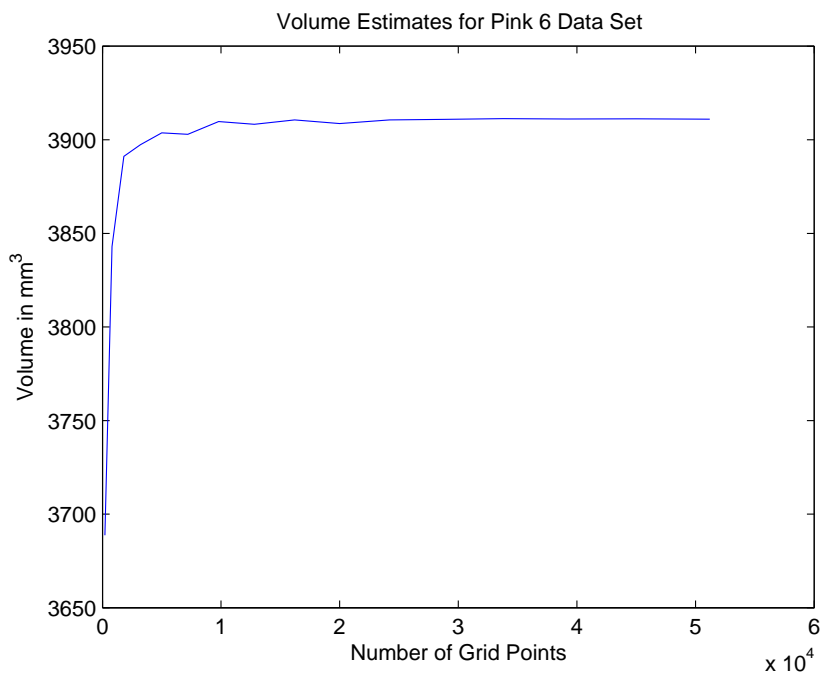

Figure 24: Volume Estimates for Pink 6 Data Set vs. Grid Size. 


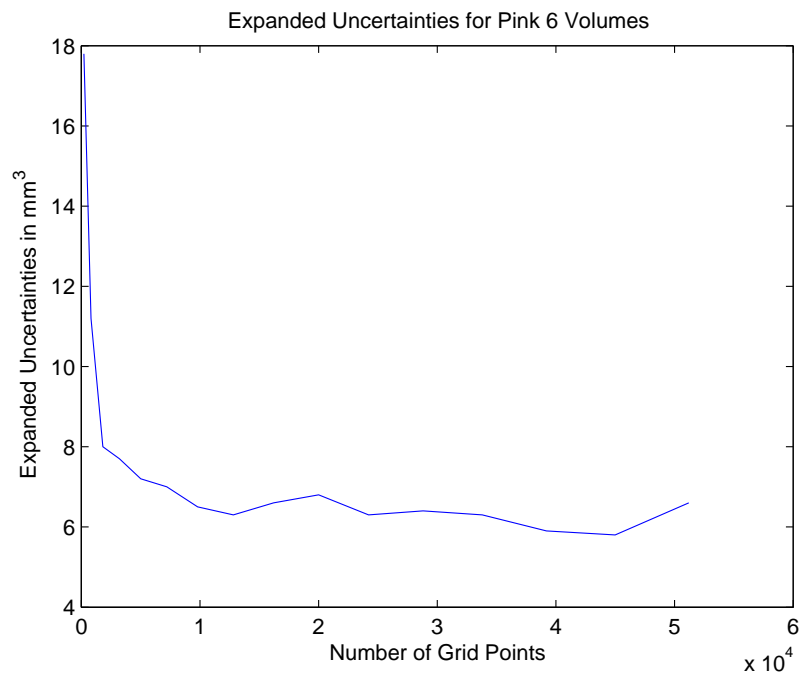

Figure 25: Uncertainty Estimates for Pink 6 Data Set vs. Grid Size.

\begin{tabular}{||c|c|c|c|c||}
\hline \hline \multicolumn{5}{||c||}{ Pink 7 Data Set } \\
\hline Grid Case & $\theta$ & $\phi$ & Volume $\left(\mathrm{mm}^{3}\right)$ & Uncertainty $\left(\mathrm{mm}^{3}\right)$ \\
\hline \hline 1 & 10 & 20 & 3697.2 & 19.2 \\
\hline 2 & 20 & 40 & 3865.3 & 10.8 \\
\hline 3 & 30 & 60 & 3897.5 & 9.0 \\
\hline 4 & 40 & 80 & 3907.6 & 7.6 \\
\hline 5 & 50 & 100 & 3913.6 & 6.8 \\
\hline 6 & 60 & 120 & 3915.9 & 6.6 \\
\hline 7 & 70 & 140 & 3917.3 & 6.2 \\
\hline 8 & 80 & 160 & 3919.2 & 6.5 \\
\hline 9 & 90 & 180 & 3919.9 & 6.2 \\
\hline 11 & 110 & 220 & 3920.8 & 6.4 \\
\hline 10 & 100 & 200 & 3920.7 & 6.6 \\
\hline 12 & 120 & 240 & 3921.1 & 6.4 \\
\hline 13 & 130 & 260 & 3921.2 & 6.6 \\
\hline 14 & 140 & 280 & 3921.7 & 6.4 \\
\hline 15 & 150 & 300 & 3921.7 & 6.3 \\
\hline 16 & 160 & 320 & 3921.9 & 6.8 \\
\hline \hline
\end{tabular}

Table 13: Estimated Volumes and Uncertainties for Pink 6 Data 


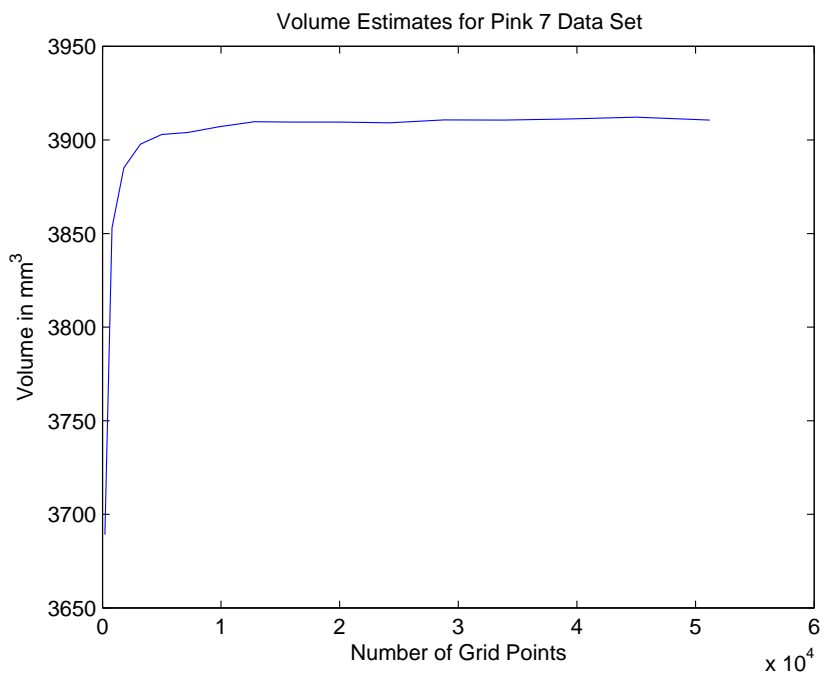

Figure 26: Volume Estimates for Pink 7 Data Set vs. Grid Size.

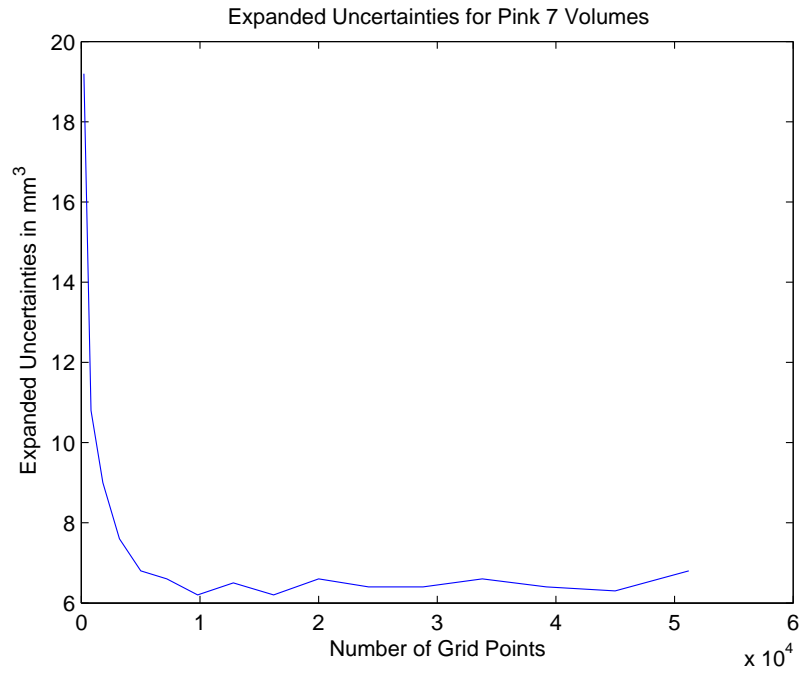

Figure 27: Uncertainty Estimates for Pink 7 Data Set vs. Grid Size. 


\begin{tabular}{||c|c|c|c|c||}
\hline \hline \multicolumn{5}{||c||}{ Green 1 Dense Data Set } \\
\hline \multicolumn{5}{||c|}{ Volumes and Expanded Uncertainties vs Grid Size } \\
\hline \hline 1 & 10 & 20 & 3816.4 & 210.6 \\
\hline 2 & 20 & 40 & 4014.0 & 135.8 \\
\hline 3 & 30 & 60 & 4052.5 & 111.6 \\
\hline 4 & 40 & 80 & 4065.4 & 105.0 \\
\hline 5 & 50 & 100 & 4067.5 & 93.6 \\
\hline 6 & 60 & 120 & 4070.6 & 94.0 \\
\hline 7 & 70 & 140 & 4071.3 & 91.4 \\
\hline 8 & 80 & 160 & 4073.0 & 91.4 \\
\hline 9 & 90 & 180 & 4073.7 & 91.4 \\
\hline 10 & 100 & 200 & 4073.5 & 89.6 \\
\hline 11 & 110 & 220 & 4074.8 & 89.8 \\
\hline 12 & 120 & 240 & 4074.5 & 88.4 \\
\hline 13 & 130 & 260 & 4075.4 & 89.2 \\
\hline 14 & 140 & 280 & 4075.0 & 87.2 \\
\hline 15 & 150 & 300 & 4075.3 & 88.0 \\
\hline 16 & 160 & 320 & 4075.2 & 87.4 \\
\hline \hline
\end{tabular}

Table 14: Estimated Volumes and Uncertainties for Green 1 Dense Data

\subsubsection{Volume Estimates and Uncertainties for the Dense Data}

In this section we report the results of volume estimates and the extended uncertainties for the dense data sets of 181 probed points on the phantom surfaces. The results do not seem to be what one would have expected. The volumes and the uncertainties have not been plotted since in general they follow the pattern established by the volumes and uncertainties in the coarse data. We will just consider the differences. The first thing of interest is that the stabilized volume estimates for the Green dense data differ from the volume estimates for the Green coarse data by about $6 \%$. This is in the negative direction in that the volume estimates for the Green dense data were smaller than for the Green coarse data. In the case of the volume estimates for the Pink dense data, the differences with the volume estimates for the Pink coarse data are approximately $4 \%$ larger. These estimates are clear from Tables 14 to 19. Another thing to notice is that the uncertainties are significantly higher as shown in Tables 14 through 19 compared to those estimated from the coarse data. In fact they appear to be an order of magnitude larger. It is not clear why the results for the dense data differ from the results for the coarse data, but we are reporting it nonetheless. The complete analysis of these differences is beyond the scope of this report but will be pursued in a coming study related to a redesign of the fitting algorithm.

\section{Summary}

The B-spline surface model joined with the Divergence Theorem seems to be a viable approach for estimating volumes of near spherical star-shaped artifacts, but the results seem to depend strongly on the distribution of the data points on the surface. In the case of sparse data the problem of extrapolating a surface fitted to the data to a grid on the surface leads potentially to unwanted 


\begin{tabular}{||c|c|c|c|c||}
\hline \hline \multicolumn{5}{|c||}{ Green 2 Dense Data Set } \\
\hline \multicolumn{5}{||c|}{ Volumes and Expanded Uncertainties vs Grid Size } \\
\hline \hline Grid Case & $\theta$ & $\phi$ & Volume $\left(\mathrm{mm}^{3}\right)$ & Uncertainty $\left(\mathrm{mm}^{3}\right)$ \\
\hline 1 & 10 & 20 & 3788.2 & 225.6 \\
\hline 2 & 20 & 40 & 3989.5 & 137.4 \\
\hline 3 & 30 & 60 & 4027.8 & 123.4 \\
\hline 4 & 40 & 80 & 4034.4 & 112.2 \\
\hline 5 & 50 & 100 & 4040.2 & 103.6 \\
\hline 6 & 60 & 120 & 4041.2 & 99.8 \\
\hline 7 & 70 & 140 & 4042.7 & 102.2 \\
\hline 8 & 80 & 160 & 4043.7 & 100.4 \\
\hline 9 & 90 & 180 & 4045.4 & 98.4 \\
\hline 10 & 100 & 200 & 4044.9 & 98.6 \\
\hline 11 & 110 & 220 & 4044.7 & 97.2 \\
\hline 12 & 120 & 240 & 4044.9 & 96.8 \\
\hline 13 & 130 & 260 & 4045.4 & 96.8 \\
\hline 14 & 140 & 280 & 4045.3 & 95.8 \\
\hline 15 & 150 & 300 & 4045.7 & 95.6 \\
\hline 16 & 160 & 320 & 4046.3 & 95.2 \\
\hline \hline
\end{tabular}

Table 15: Estimated Volumes and Uncertainties for Green 2 Dense Data

\begin{tabular}{||c|c|c|c|c||}
\hline \hline \multicolumn{5}{||c||}{ Green 3 Dense Data Set } \\
\hline Grid Case & $\theta$ & $\phi$ & Volume $\left(\mathrm{mm}^{3}\right)$ & Uncertainty $\left(\mathrm{mm}^{3}\right)$ \\
\hline \hline 1 & 10 & 20 & 3859.8 & 225.0 \\
\hline 2 & 20 & 40 & 4064.0 & 138.0 \\
\hline 3 & 30 & 60 & 4097.9 & 130.4 \\
\hline 4 & 40 & 80 & 4102.7 & 115.4 \\
\hline 5 & 50 & 100 & 4110.2 & 105.6 \\
\hline 6 & 60 & 120 & 4113.2 & 105.6 \\
\hline 7 & 70 & 140 & 4114.8 & 105.4 \\
\hline 8 & 80 & 160 & 4116.3 & 100.2 \\
\hline 9 & 90 & 180 & 4119.4 & 100.6 \\
\hline 10 & 100 & 200 & 4119.1 & 101.6 \\
\hline 11 & 110 & 220 & 4118.6 & 99.4 \\
\hline 12 & 120 & 240 & 4118.6 & 99.8 \\
\hline 13 & 130 & 260 & 4119.7 & 99.6 \\
\hline 14 & 140 & 280 & 4118.7 & 99.2 \\
\hline 15 & 150 & 300 & 4120.0 & 98.0 \\
\hline 16 & 160 & 320 & 4119.3 & 99.6 \\
\hline \hline
\end{tabular}

Table 16: Estimated Volumes and Uncertainties for Green 3 Dense Data 


\begin{tabular}{||c|c|c|c|c||}
\hline \hline \multicolumn{5}{||c||}{ Pink 1 Dense Data Set } \\
\hline \multicolumn{5}{||c|}{ Volumes and Expanded Uncertainties vs Grid Size } \\
\hline \hline Grid Case & $\theta$ & $\phi$ & Volume $\left(\mathrm{mm}^{3}\right)$ & Uncertainty $\left(\mathrm{mm}^{3}\right)$ \\
\hline 1 & 10 & 20 & 3663.7 & 193.0 \\
\hline 2 & 20 & 40 & 3847.9 & 105.6 \\
\hline 3 & 30 & 60 & 3878.9 & 88.0 \\
\hline 4 & 40 & 80 & 3891.9 & 83.8 \\
\hline 5 & 50 & 100 & 3896.0 & 75.2 \\
\hline 6 & 60 & 120 & 3898.5 & 73.2 \\
\hline 7 & 70 & 140 & 3902.3 & 73.2 \\
\hline 8 & 80 & 160 & 3903.0 & 72.0 \\
\hline 9 & 90 & 180 & 3903.2 & 72.0 \\
\hline 10 & 100 & 200 & 3903.8 & 71.8 \\
\hline 11 & 110 & 220 & 3905.0 & 71.0 \\
\hline 12 & 120 & 240 & 3905.0 & 70.8 \\
\hline 13 & 130 & 260 & 3905.2 & 69.2 \\
\hline 14 & 140 & 280 & 3904.7 & 69.6 \\
\hline 15 & 150 & 300 & 3905.5 & 69.2 \\
\hline 16 & 160 & 320 & 3905.3 & 69.0 \\
\hline \hline
\end{tabular}

Table 17: Estimated Volumes and Uncertainties for Pink 1 Dense Data

\begin{tabular}{||c|c|c|c|c||}
\hline \hline \multicolumn{5}{||c||}{ Pink 2 Dense Data Set } \\
\hline \multicolumn{5}{||c|}{ Volumes and Expanded Uncertainties vs Grid Size } \\
\hline \hline Grid Case & $\theta$ & $\phi$ & Volume $\left(\mathrm{mm}^{3}\right)$ & Uncertainty $\left(\mathrm{mm}^{3}\right)$ \\
\hline 1 & 10 & 20 & 3690.2 & 191.8 \\
\hline 2 & 20 & 40 & 3881.2 & 110.6 \\
\hline 3 & 30 & 60 & 3916.1 & 91.4 \\
\hline 4 & 40 & 80 & 3923.5 & 83.6 \\
\hline 5 & 50 & 100 & 3926.6 & 73.6 \\
\hline 6 & 60 & 120 & 3932.6 & 74.4 \\
\hline 7 & 70 & 140 & 3934.1 & 74.8 \\
\hline 8 & 80 & 160 & 3934.8 & 73.6 \\
\hline 9 & 90 & 180 & 3935.8 & 71.8 \\
\hline 10 & 100 & 200 & 3934.2 & 71.2 \\
\hline 11 & 110 & 220 & 3935.6 & 71.0 \\
\hline 12 & 120 & 240 & 3935.8 & 70.4 \\
\hline 13 & 130 & 260 & 3936.3 & 70.0 \\
\hline 14 & 140 & 280 & 3936.1 & 69.0 \\
\hline 15 & 150 & 300 & 3936.1 & 70.4 \\
\hline 16 & 160 & 320 & 3936.1 & 70.0 \\
\hline \hline
\end{tabular}

Table 18: Estimated Volumes and Uncertainties for Pink 2 Dense Data 


\begin{tabular}{||c|c|c|c|c||}
\hline \hline \multicolumn{6}{||c||}{ Pink 3 Dense Data Set } \\
\hline \multicolumn{5}{||c|}{ Volumes and Expanded Uncertainties vs Grid Size } \\
\hline \hline Grid Case & $\theta$ & $\phi$ & Volume $\left(\mathrm{mm}^{3}\right)$ & Uncertainty $\left(\mathrm{mm}^{3}\right)$ \\
\hline 2 & 10 & 20 & 3274.6 & 236.8 \\
\hline 3 & 20 & 40 & 3462.9 & 153.6 \\
\hline 4 & 30 & 60 & 3488.5 & 130.8 \\
\hline 5 & 40 & 80 & 3496.4 & 118.6 \\
\hline 6 & 50 & 100 & 3498.3 & 110.8 \\
\hline 7 & 60 & 120 & 3500.4 & 110.4 \\
\hline 8 & 70 & 140 & 3502.8 & 108.0 \\
\hline 9 & 80 & 160 & 3503.2 & 106.8 \\
\hline 10 & 90 & 180 & 3505.9 & 105.2 \\
\hline 11 & 100 & 200 & 3504.5 & 105.4 \\
\hline 12 & 110 & 220 & 3506.2 & 104.0 \\
\hline 13 & 130 & 240 & 3506.2 & 103.0 \\
\hline 14 & 140 & 260 & 3507.5 & 103.2 \\
\hline 15 & 150 & 300 & 3508.2 & 104.2 \\
\hline 16 & 160 & 320 & 3507.5 & 103.4 \\
\hline \hline
\end{tabular}

Table 19: Estimated Volumes and Uncertainties for Pink 3 Dense Data

oscillations in the neighborhood of some of the sparse data points. In the current algorithm this problem was dealt with for convenience by a chopping operation, but this may not be a good procedure and may have affected the differences between the coarse data results and the dense data results, although this needs to be investigated further.

The results obtained from the coarse data distribution appear to be consistent with the results from the spherical model fits. As shown in Tables 8, 9, and 10 in Section 4.6.2 the volume estimates for the Green sphere are very close to the estimates obtained by the B-spline model suggesting that the Green sphere is essentially spherical in shape. As expected the volume estimates in Table 4 for the Pink sphere are significantly lower than the B-spline model estimates in Tables 11, 12, and 11, in this case suggesting that the Pink sphere has a significant non-spherical shape.

The results from the dense data distribution were not what would have been expected. The uncertainties were significntly higher than those for the coarse data. Tables 14 to 16 show volumes smaller than those from 8, 9, and 10. Tables 17 and 18 show similar volume estimates but Table 19 shows significantly lower volume estimates. This, of course, could have been brought about by any difficulty in holding the Pink phantom in position 3.

In summary we conclude that the combination of using the CMM to obtain surface data and the B-spline/Divergence Theorem volume estimation method can produce useful results but that there are some limitations. First, the CMM is primarily used for probing manufactured metalic artifacts and its use in probing non-metalic artifacts such as the FDA phantoms can lead to some large uncertainties in volume estimation. Second, the distribution of probed points can lead to unexpected results. So there is a problem of consistency here. Third, the methods used may not provide useful volume estimation for more complex artifacts that surely would arise in developing simulated lung cancers. The current artifacts were near-spherical and even these did not provide fully expected results based on data distribution. As a result of these limitations we think other means of estimating "ground truth" volumes for the current and any future phantoms be investigated 
by the FDA.

\section{Acknowledgement}

The authors wish to acknowledge the generous assistance of Stefan Leigh of the Statistical Engineering Division of ITL at NIST in designing the volume uncertainty estimation algorithm by the bootstrap method. We also wish to acknowledge the gracious assistance of Prof. Dianne P. O'Leary, University of Maryland, in writing an efficient version of the least squares program using SVD. Finally, we acknowledge the use of the Nelder-Mead code by Prof. Timothy Sauer, George Mason University.

\section{Disclaimer}

Certain commercial software products are identified in this paper in order to adequately specify the computational procedures. Such identification does not imply recommendation or endoresement by the National Institute of Standards and Technology nor does it imply that the software products identified are necessarily the best available for the purpose.

\section{References}

[1] American Cancer Society, Cancer Facts \& Figures 200\%. Atlanta: American Cancer Society, 2007.

[2] Cox, M. G., 'The Numerical Evaluation of B-splines', J. Inst. Maths Applics, 10, (1972), 134149.

[3] de Boor, C., A Practical Guide to Splines, Springer-Verlag, New York, 1978.

[4] Dierckx, P., 'An Algorithm for Surface-Fitting with Spline Functions', IMA Journal of Numerical Analysis, 1, (1981), 267-283.

[5] Dierckx, P., 'Algorithms for Smoothing Data on the Sphere with Tensor Product Splines', Computing, 32, 319-342.

[6] Dierckx, P., Curve and Surface Fitting with Splines, Clarendon Press, Oxford, 2006.

[7] Draper, N. and Smith, H., Applied Regression Analysis, John Wiley \& Sons, New York, 1981.

[8] Eberly, D. H., Game Physics, Morgan Kaufmann Publishers, Amsterdam, 2004.

[9] Efron, B. and Tibshirani, R. J.,textitAn Introduction to the Bootstrap, Chapman and Hall, New York, 1993.

[10] Hayes, J. G. and Halliday, J., 'The Least-squares Fitting of Cubic Spline Surfaces to General Data Sets', J. Inst. Maths Applics, 14, (1974), 89-103.

[11] http://en.wikipedia.org/wiki/Computer_tomography

[12] Jenal, A., Siegel, R., Ward, E., Murray, T., Xu, J., Thun, J., 'Cancer Statistics, 2007', CA Cancer J Clin, 57, 1,(2007), 43-66. 
[13] Rogers, D. F. and Adams, J. A., Mathematical Elements for Computer Graphics, McGraw-Hill, Boston, 1990.

[14] Sauer, T., Numerical Analysis, Pearson, Boston, 2006.

[15] Schneider, P. J. and Eberly, D. H., Geometric Tools for Computer Graphics, Morgan Kaufmann Publishers, Amsterdam, 2003.

[16] Shakarji, C. M., 'Least-Squares Fitting Algorithms of the NIST Algorithm Testing System', $J$. Res. Natl. Inst. Stand. Technol., 103, (1998), 633-641.

[17] Taylor, A. E., Advanced Calculus, Ginn and Company, Boston, 1955.

[18] Taylor, B. N. and Kuyatt, C. E., 'Guidelines for Evaluating and Expressing the Uncertainty of NIST Measurement Results', NIST Technical Note 1297, 1994 Edition, National Institute of Stadards and Technology, 1994.

[19] Therasse, P., Arbuck, S. G., Eisenhauer, E. A., Wanders, J., Kaplan, R. S., Rubinstein, L., Verweij, J. Van Glabbeke, M., van Oosterom, A. T., Christian, M. C., Gwyther, S. G., 'New Guidelines to Evaluate the Response to Treatment in Solid Tumors', Journal of the National Cancer Institute, 92, 3, (2000), 205-216.

\section{A Fitting a Sphere to Probe Data}

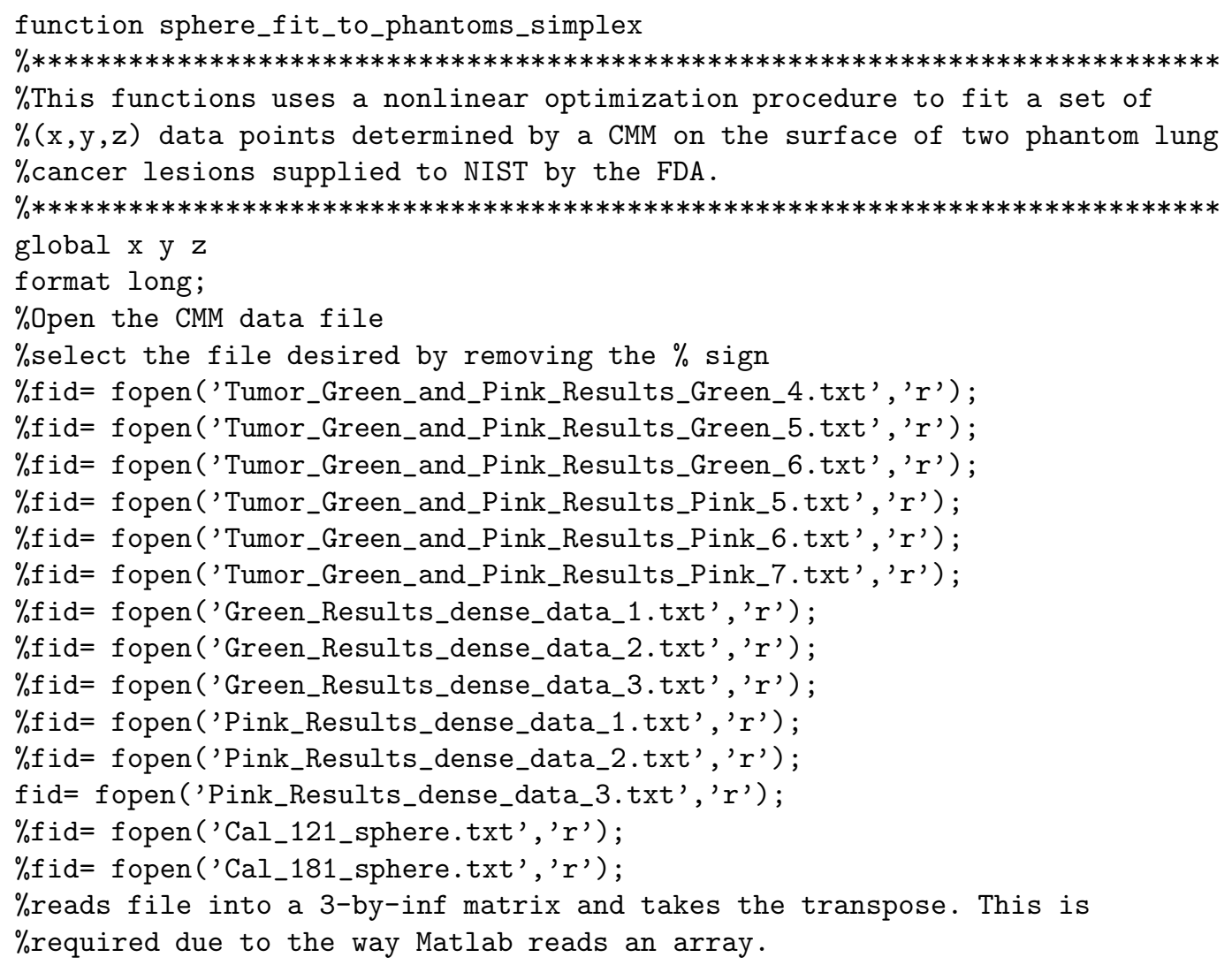




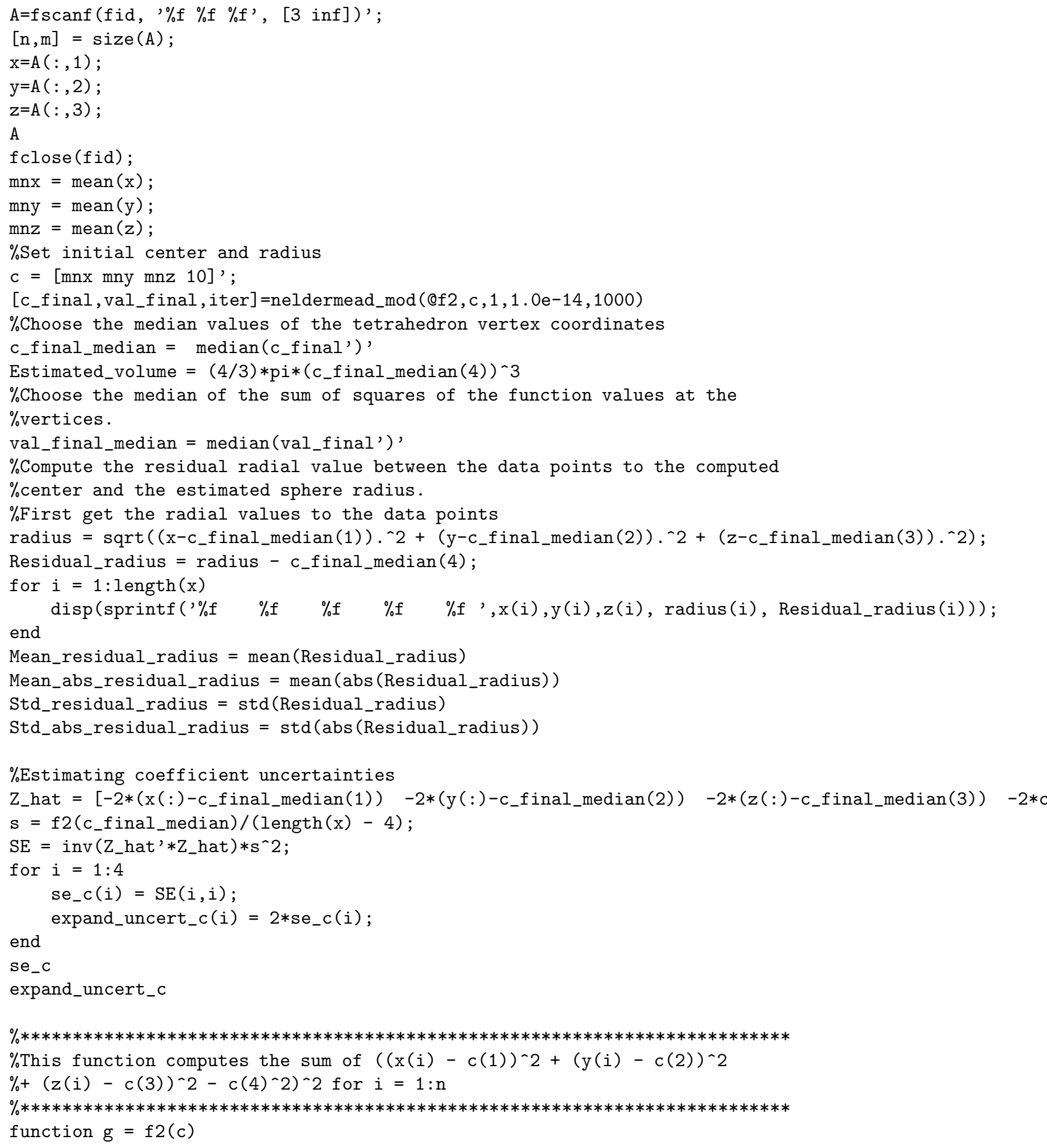




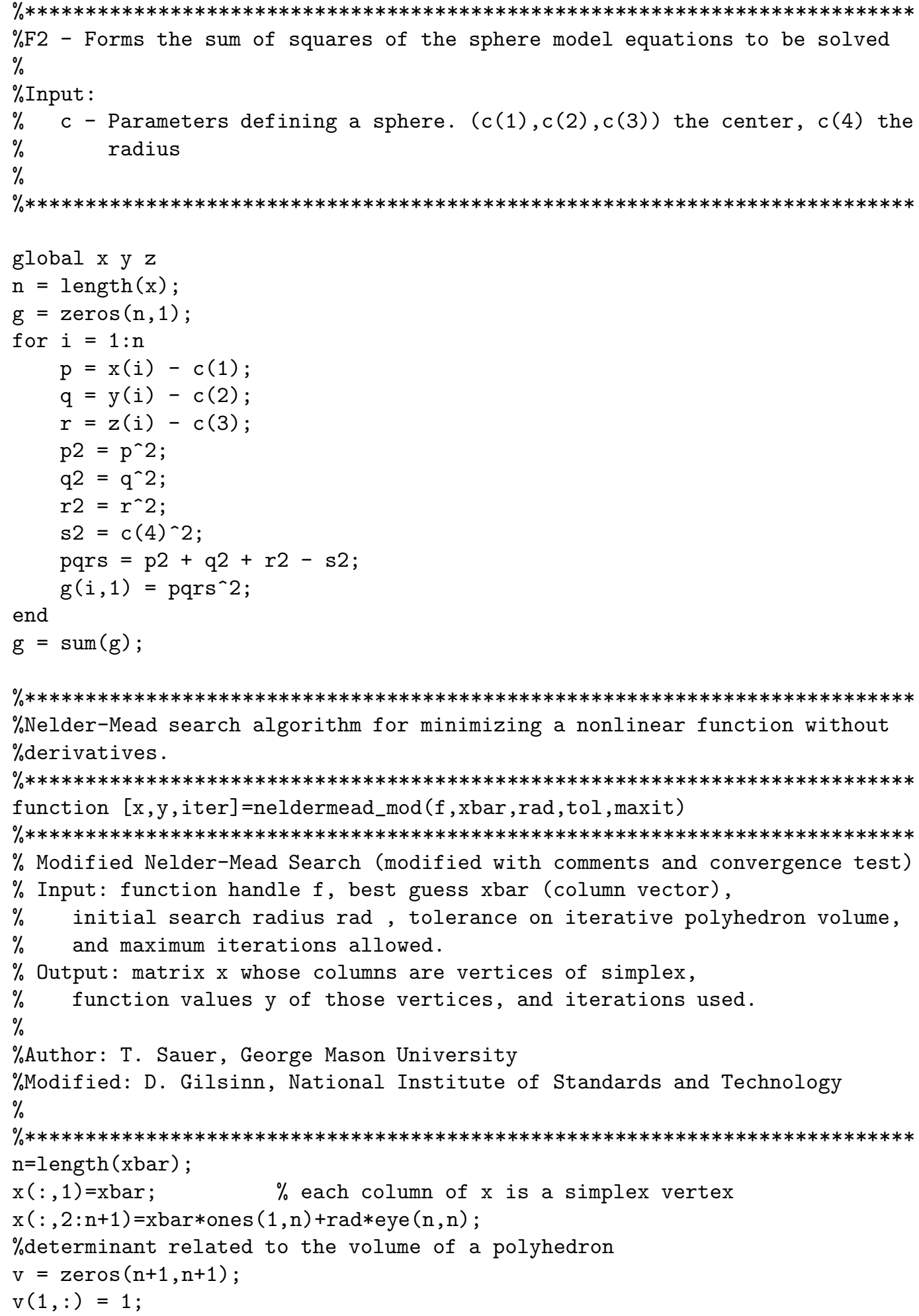




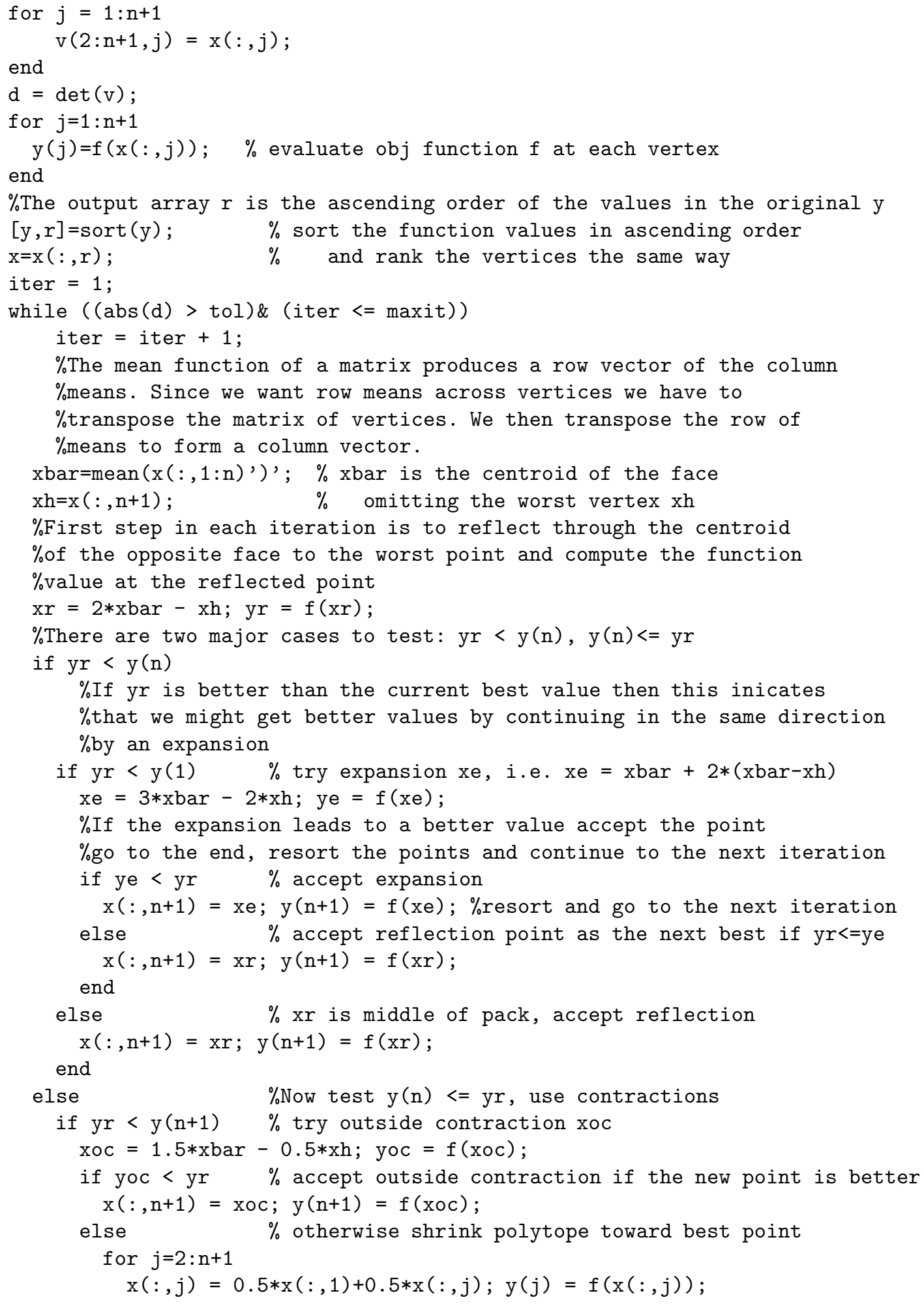




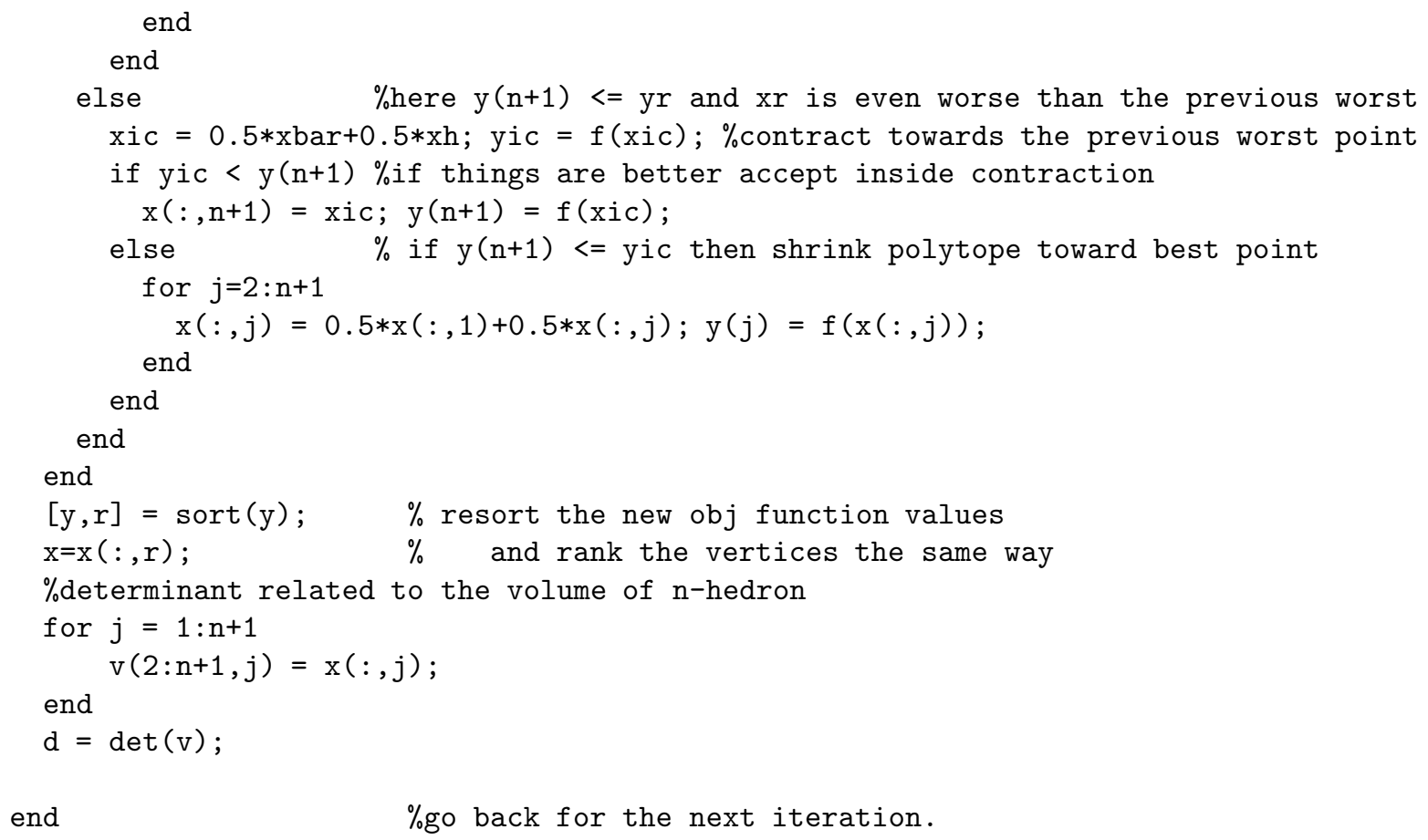

\section{B Main Volume Estimation Code}

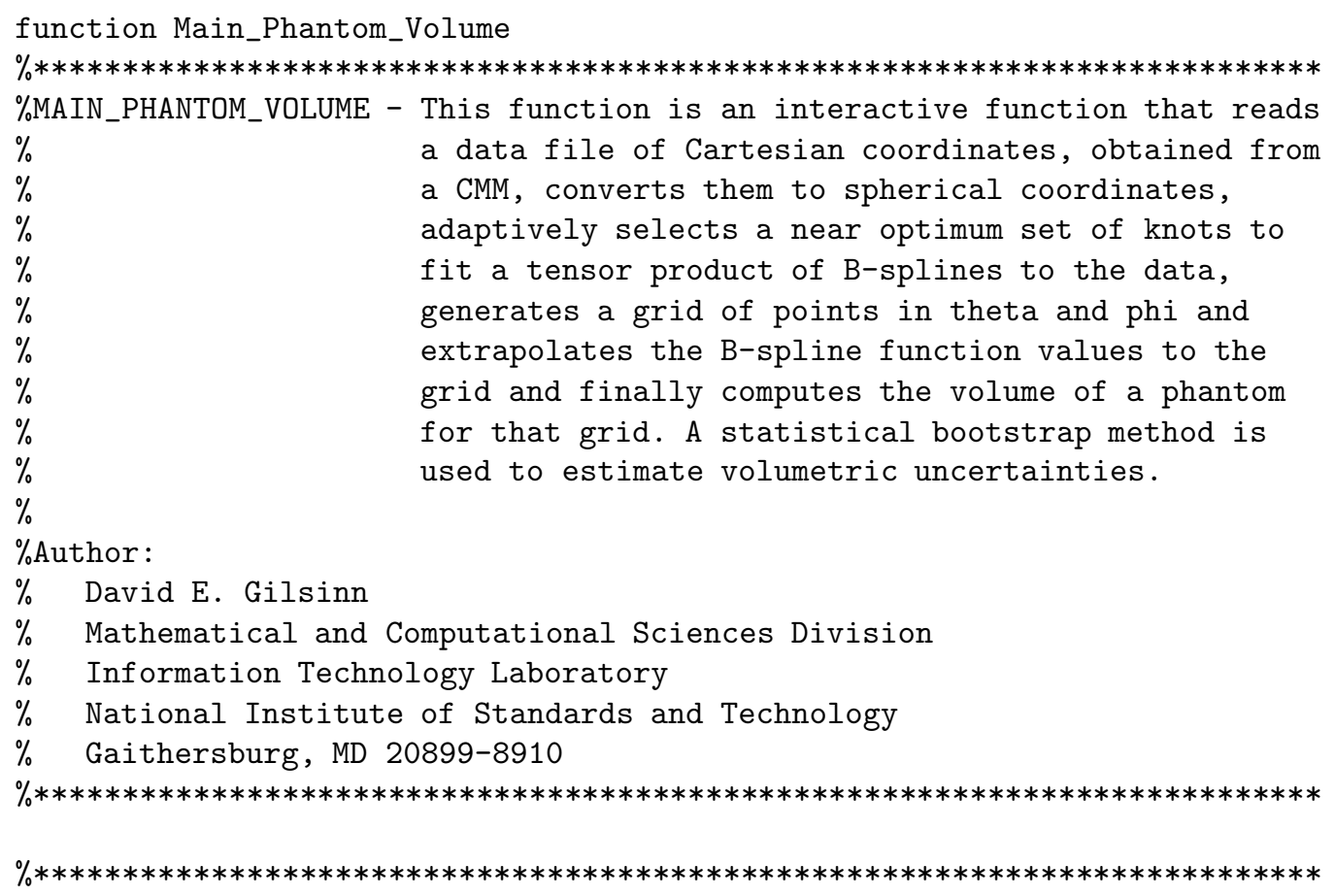


$\%$ Data initialization section, knot selection, and least squares fit. $\%$ Executed once.

$\% * * * * * * * * * * * * * * * * * * * * * * * * * * * * * * * * * * * * * * * * * * * * * * * * * * * * * * * * * * * * * * * * * * * * * * * * * * * * *$

$\%$ Get the Cartesian data. Choose a file name included in the function

$[\mathrm{x}, \mathrm{y}, \mathrm{z}]=$ Get_CMM_data;

$\mathrm{N}=\operatorname{length}(\mathrm{x})$;

$\%$ Convert to spherical coordinates. The term base is used to indicate

$\%$ results not affected by any scaling of the radial data, otherwise the term

$\%$ shift will be used.

[theta_base,phi_base,rad_base] = Get_Spherical_Coordinates $(\mathrm{x}, \mathrm{y}, \mathrm{z})$;

$\%$ Take the mean out of the radial data. It will be added back later.

mean_rad_base $=$ mean (rad_base) ;

max_rad_base $=\max ($ rad_base $)$;

min_rad_base $=\min ($ rad_base $)$;

$\%$ rad_shift $=$ rad_base - mean_rad_base;

rad_shift $=$ rad_base;

\%Adaptively select an optimum set of knots for theta and phi

$\%$ The final fit is made to the shifted radial data.

[rad_pred_shift,c_shift, NM_shift,resid_shift,knts_theta,knts_phi,iter]...

= adaptive_knot_selection(theta_base,phi_base,rad_shift);

$\operatorname{disp}\left(' * * * * * * * * * * * * * * * * * * * * * * * * * * * * * * * * * * * * * * * * * * * * * *^{\prime}\right)$ );

disp('Initial Fitting Statistics');

$\operatorname{disp}(' * * * * * * * * * * * * * * * * * * * * * * * * * * * * * * * * * * * * * * * * * * * * * * ')$ );

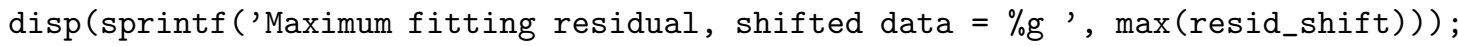

disp(sprintf('Minimum of fitting residual, shifted data $=\% \mathrm{~g}, \min \left(\mathrm{resid}_{-}\right.$shift)));

disp(sprintf('Mean of fitting residual, shifted data $=\%$ g ',mean(resid_shift))) ;

disp(sprintf('Standard deviation of fitting residual, shifted data $=\%$ g ',std(resid_shift)));

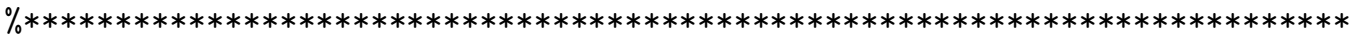

$\%$ End data initialization, knot selection, and initial least squares fit

$\% * * * * * * * * * * * * * * * * * * * * * * * * * * * * * * * * * * * * * * * * * * * * * * * * * * * * * * * * * * * * * * * * * * * * * * * * * * * * *$

\% $* * * * * * * * * * * * * * * * * * * * * * * * * * * * * * * * * * * * * * * * * * * * * * * * * * * * * * * * * * * * * * * * * * * * * * * * * * *)$

\% Begin interactive volume determination for selected grid sizes

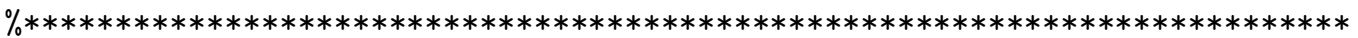

$\operatorname{disp}(' * * * * * * * * * * * * * * * * * * * * * * * * * * * * * * * * * * * * * * * * * * * * * * *$ ') )

disp('Interactive Grid Selection and Volume Estimate');

$\operatorname{disp}(' * * * * * * * * * * * * * * * * * * * * * * * * * * * * * * * * * * * * * * * * * * * * * * *)$ ');

$f \operatorname{lag}=1$; 
while ( $f$ lag $>0$ )

flag $=$ input ('To continue with a new grid type 1 , else type $-1>$ ');

if ( $f$ lag $<=0$ )

break;

end

$\%$ Set up grid parameters for generating a longitudinal and latitudinal grid

nt_grid = input ('Enter desired number of theta grid points $>$ ');

np_grid = input('Enter desired number of phi grid points $>$ ');

$\%$ This function adjusts the extrapolation due to the shifted

$\%$ coefficients by rescaling the predicted radial values on the grid.

[t_grid,p_grid,rad1_base, N1M1]...

= Generate_grid_data(c_shift,nt_grid,np_grid, knts_theta,knts_phi,rad_base);

$\%$ Compute the volume

$\operatorname{vol}(1)=$ volume (t_grid,p_grid,rad1_base);

disp(sprintf('Initial volume in cubic $\mathrm{mm}=\% \mathrm{~g}$ ', vol(1)));

Boot $=1$;

Boot $=$ input ('Type -1 to skip the bootstrap process $>$ ');

new_resid $=\operatorname{zeros}(\mathrm{N}, 1)$;

data_tol $=1 \mathrm{e}-14$;

if (Boot > 0)

\%Botstrap process to estimate volume uncertainty and ref volume

for $i=2: 200$

disp(sprintf('Bootstrap iteration \%d out of 200.',i));

for $\mathrm{j}=1: \mathrm{N}$

$\mathrm{u}=$ rand;

$i i=f i x(u * N)+1$;

new_resid $(j)=$ resid_shift(ii);

end

new_pred_shift $=$ rad_pred_shift + new_resid;

$\%$ pred values are the extrapolated values to the grid based on

$\%$ the new coefficients $c$

c1 = 1sq_2D_spline (NM_shift, new_pred_shift,data_tol);

pred $=$ N1M1 $*$ c1 + mean_rad_base;

delta_plus $=$ max_rad_base - mean_rad_base;

delta_minus $=$ mean_rad_base - min_rad_base;

outlyer $=$ find $($ (pred $<$ mean_rad_base - delta_minus $) \mid$ (pred $>$ mean_rad_base + delta_pl

rnd $=$ rand (length (outlyer), 1 );

for $i \mathrm{i}=1$ : length (outlyer)

$j=$ outlyer $(i i)$;

if $(\operatorname{rnd}(\mathrm{ii}, 1)<=0.5)$

$\operatorname{pred}(j)=$ mean_rad_base - rnd $(i i, 1) *$ delta_minus;

else

$\operatorname{pred}(j)=$ mean_rad_base + rnd $(i i, 1) *$ delta_plus;

end

end

$\operatorname{vol}(i)=$ volume (t_grid,p_grid,pred);

$\%$ disp (sprintf ('Volume $(\% d)=\%$ f', i, vol (i))) ;

end 
vol_uncert $=\operatorname{std}(\mathrm{vol})$;

vol_ref $=\operatorname{mean}(\mathrm{vol})$;

disp(sprintf ('Volume uncertainty = \% f cubic mm', vol_uncert)) ;

end

disp(sprintf('Reference Volume = \%f cubic mm', vol_ref)) ;

$\%$ clear variables for next pass

clear t_grid p_grid rad1_base

end

\section{Main_Phantom_Volume Support Programs}

\section{C.1 Function to Read CMM Data}

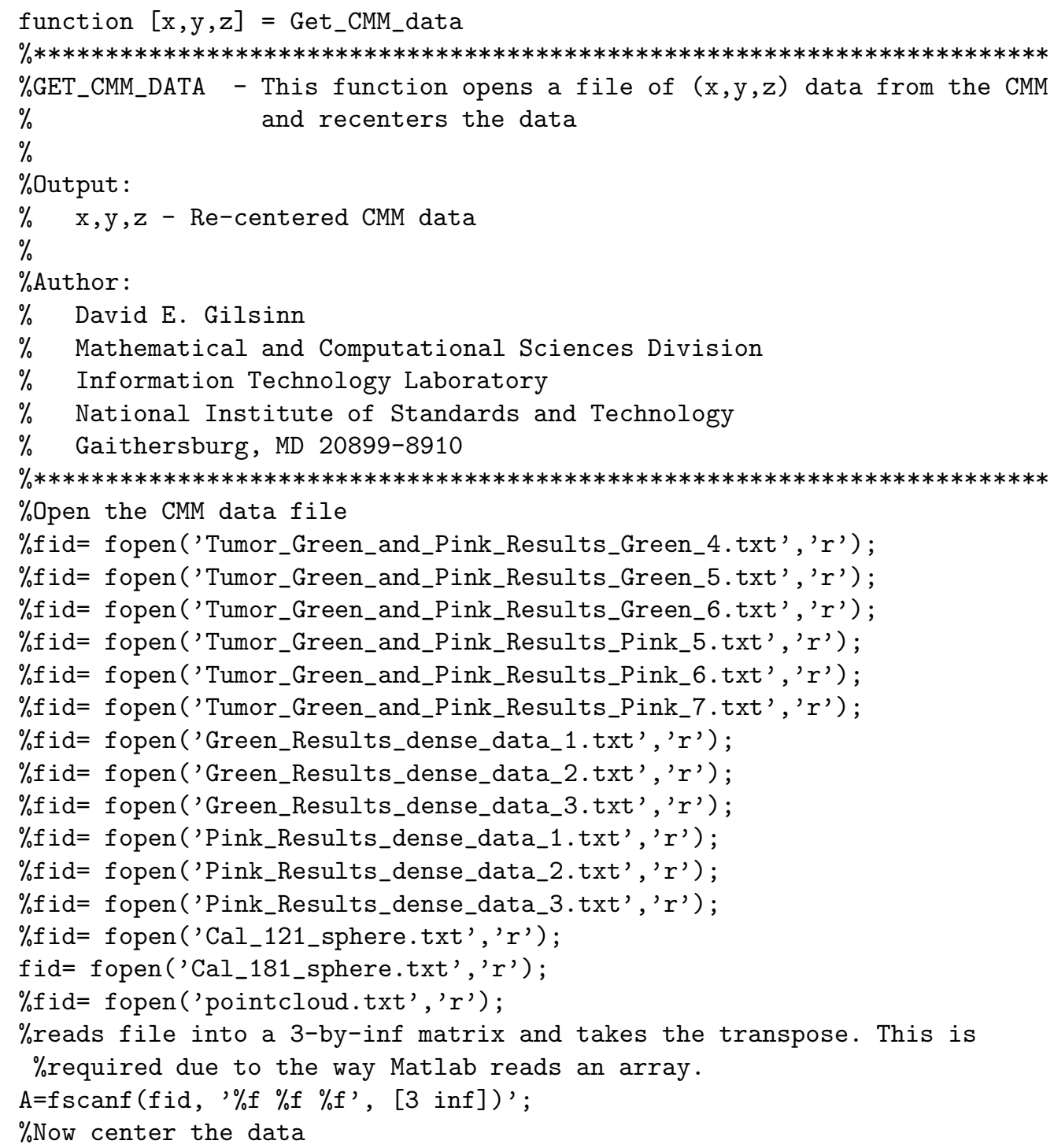




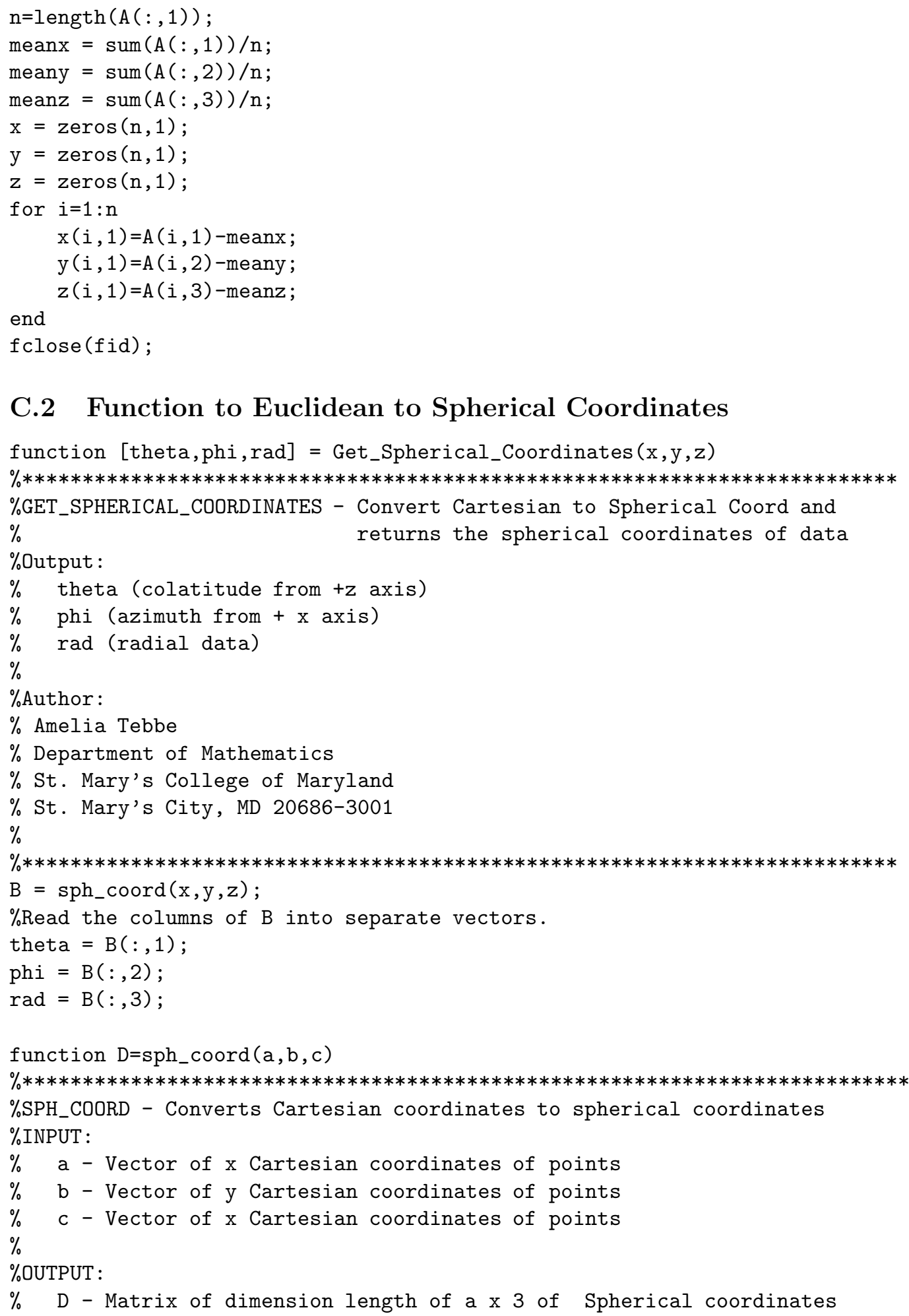

C.2 Function to Euclidean to Spherical Coordinates 


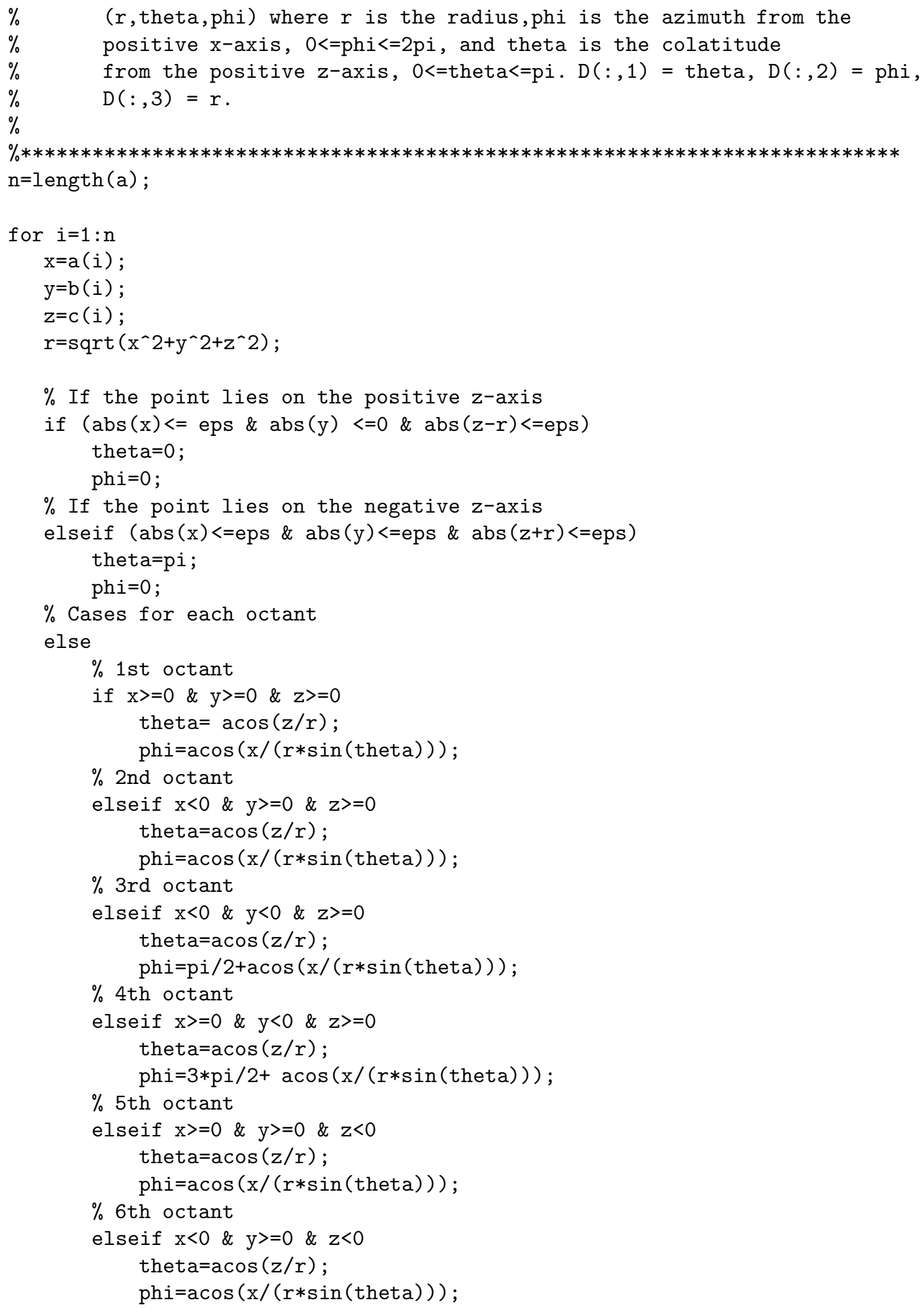




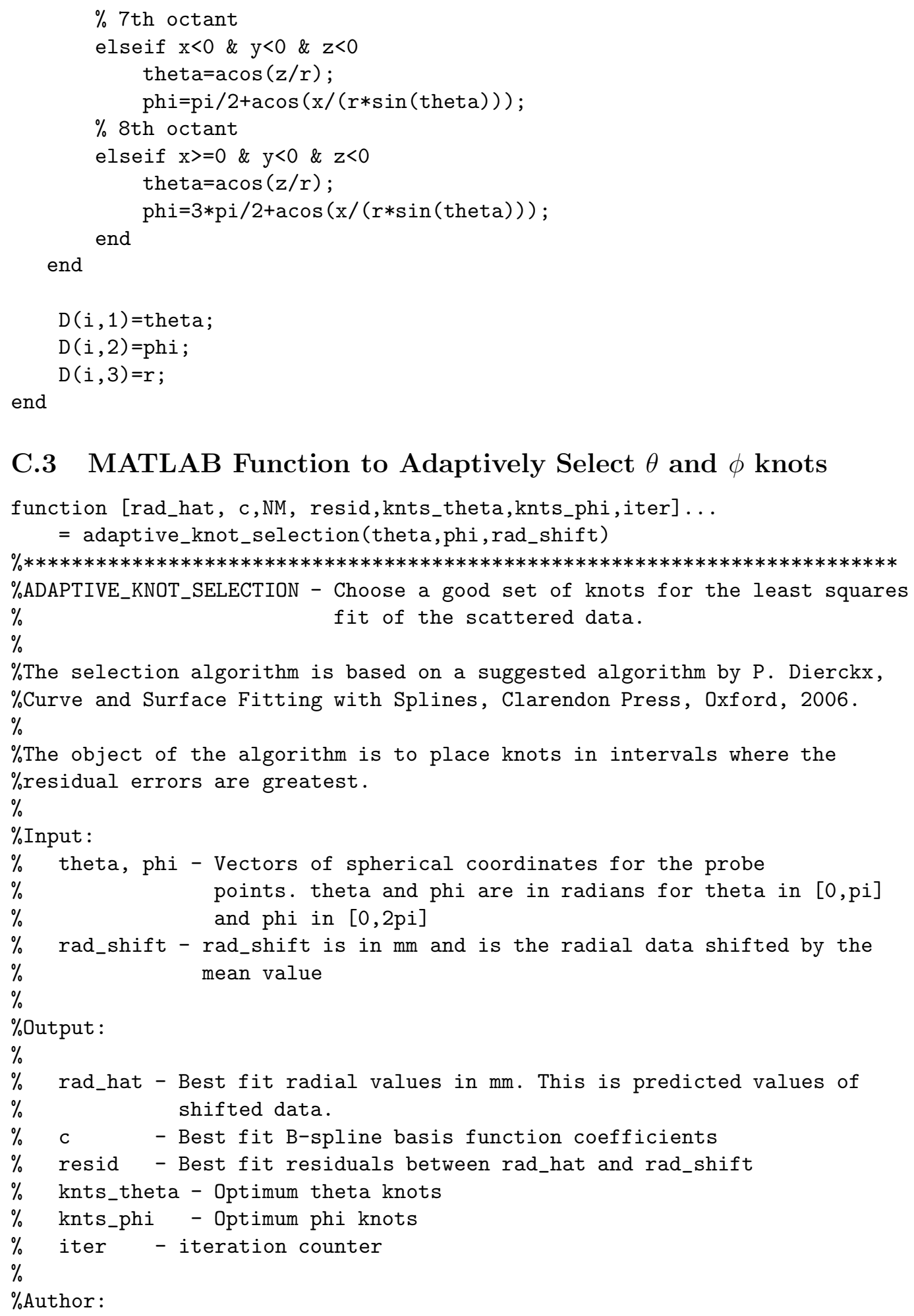

\section{C.3 MATLAB Function to Adaptively Select $\theta$ and $\phi$ knots}

function [rad_hat, c,NM, resid,knts_theta,knts_phi,iter]...

= adaptive_knot_selection(theta,phi,rad_shift) 
$\% \quad$ David E. Gilsinn

$\% \quad$ Mathematical and Computational Sciences Division

$\%$ Information Technology Laboratory

$\% \quad$ National Institute of Standards and Technology

$\% \quad$ Gaithersburg, MD 20899-8910

$\%$

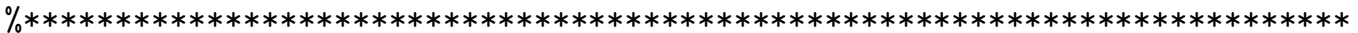

$\%$ Set a tolerance for computing the pseudo inverse for diagonal matrix $\mathrm{S}$ in $\%$ the Singular Value Decomposition (SVD) solution of the least squares $\%$ problem.

data_tol $=1 \mathrm{e}-14$;

$\%$ set initial knots with 4 evenly spaced in theta and 8 in phi

knts_theta $=\left[\begin{array}{llllll}0.0 & 1.3 & 1.7 & 2.0 & 3 & \text { pi }\end{array}\right]^{\prime} ; \%$ Specified knots for theta

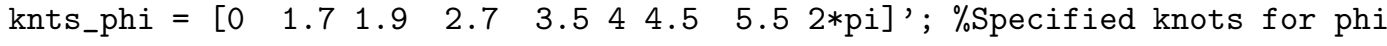

$\%$ compute the fit for the initial data set and define the R_squared

$\%$ statistic

NM = tensor_prod_spl(theta,phi,knts_theta,knts_phi); \%Get tensor product

$c=$ lsq_2D_spline (NM,rad_shift,data_tol); \%Do a least squares fit by SVD

$\%$ Get the initial residual

resid = abs (NM*c-rad_shift);

$\%$ Compute the initial predicted values

rad_hat $=\mathrm{NM} * \mathrm{C}$;

$\%$ Compute the initail $\mathrm{R}$ squared statistic (coefficient of determination)

rad_bar = mean (rad_shift);

R_square $=\left(\operatorname{sum}\left(\left(\right.\right.\right.$ rad_hat $\left.\left.\left.-\operatorname{rad} \_b a r\right) .{ }^{` 2}\right)\right) /\left(\operatorname{sum}\left(\left(\operatorname{rad} \_s h i f t-r a d \_b a r\right) . ` 2\right)\right)$

$\%$ Compute the degrees of freedom

n_dat $=$ length (rad_shift);

$\mathrm{df}=\mathrm{n}_{-}$dat $-\operatorname{length}(\mathrm{c})$;

$\%$ Initialize iteration counter

iter $=1$;

while (R_square $<0.98) \&(\mathrm{df}>=1)$

iter $=$ iter $+1 ; \%$ Update iteration counter

$\%$ Begin the automatic knot adjustment algorithm

$\%$ Compute the sum of squares of the residuals of the fit to all points in

$\%$ the interval (knts_theta(i),knts_theta(i+1))

lkt = length (knts_theta);

for $i=1: 1 \mathrm{kt}-1$

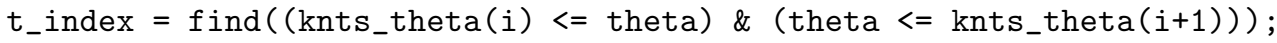

Res $=$ resid(t_index);

$\operatorname{del}{ }_{-} t(i)=(\operatorname{norm}(\operatorname{Res}))^{\wedge} 2$;

end

$\%$ del_t

$\mathrm{u}=\mathrm{f}$ ind $\left(\left(\right.\right.$ del_t $\left.\left.>=\max \left(\mathrm{del}_{-} \mathrm{t}\right)\right)\right) ; \%$ Get the maximum of the sums for theta

$\%$ Compute the sum of squares of the residuals of the fit to all points in

$\%$ the interval (knts_phi(i),knts_phi(i+1))

$1 \mathrm{kp}=$ length (knts_phi);

for $i=1: 1 \mathrm{kp}-1$

p_index $=$ find $\left(\left(\operatorname{knts}_{-}\right.\right.$phi $(i)<=$ phi) \& (phi $\left.\left.<=\operatorname{knts} \_p h i(i+1)\right)\right)$; 


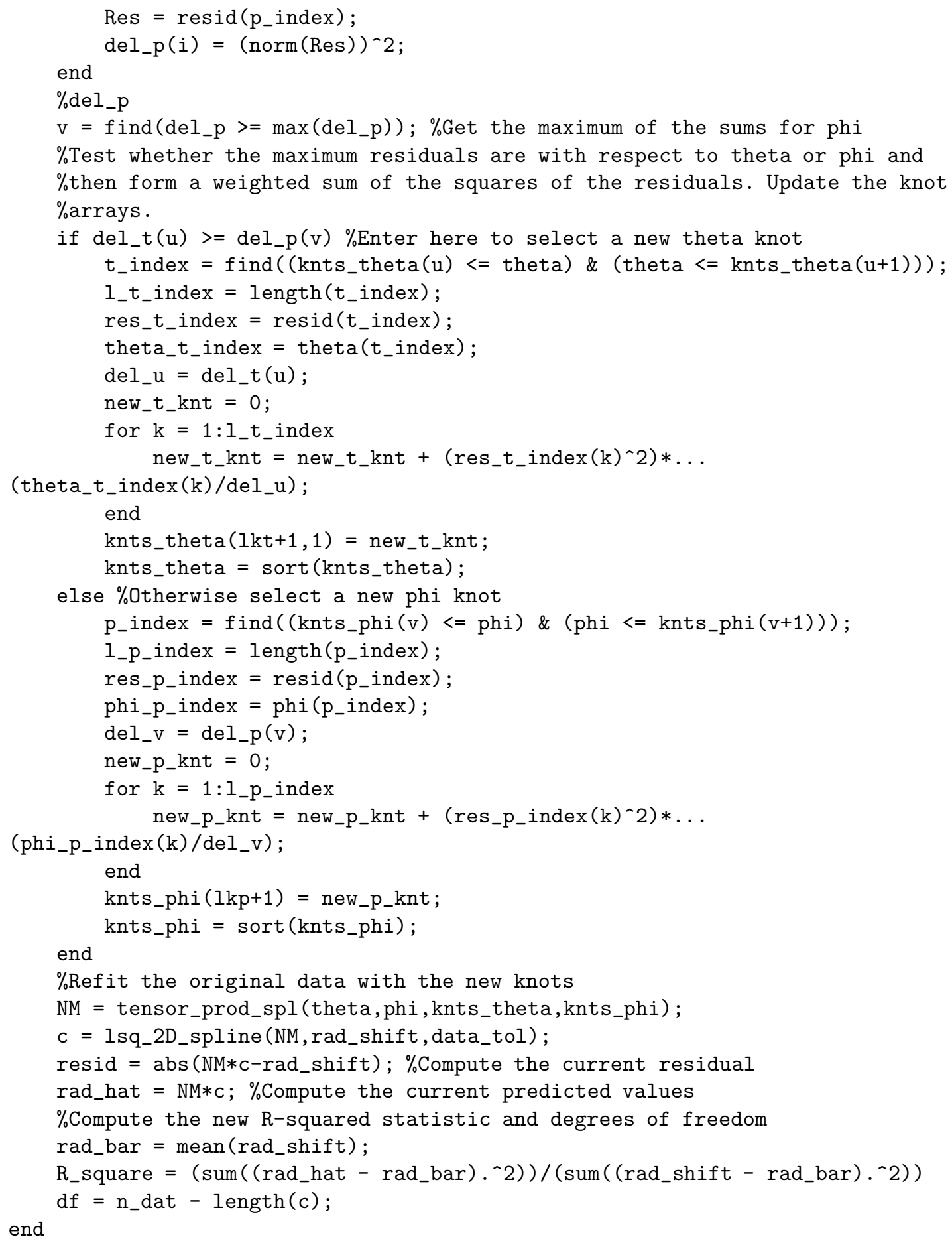




\section{C.4 Function to Compute the Tensor Product of Two B-splines}

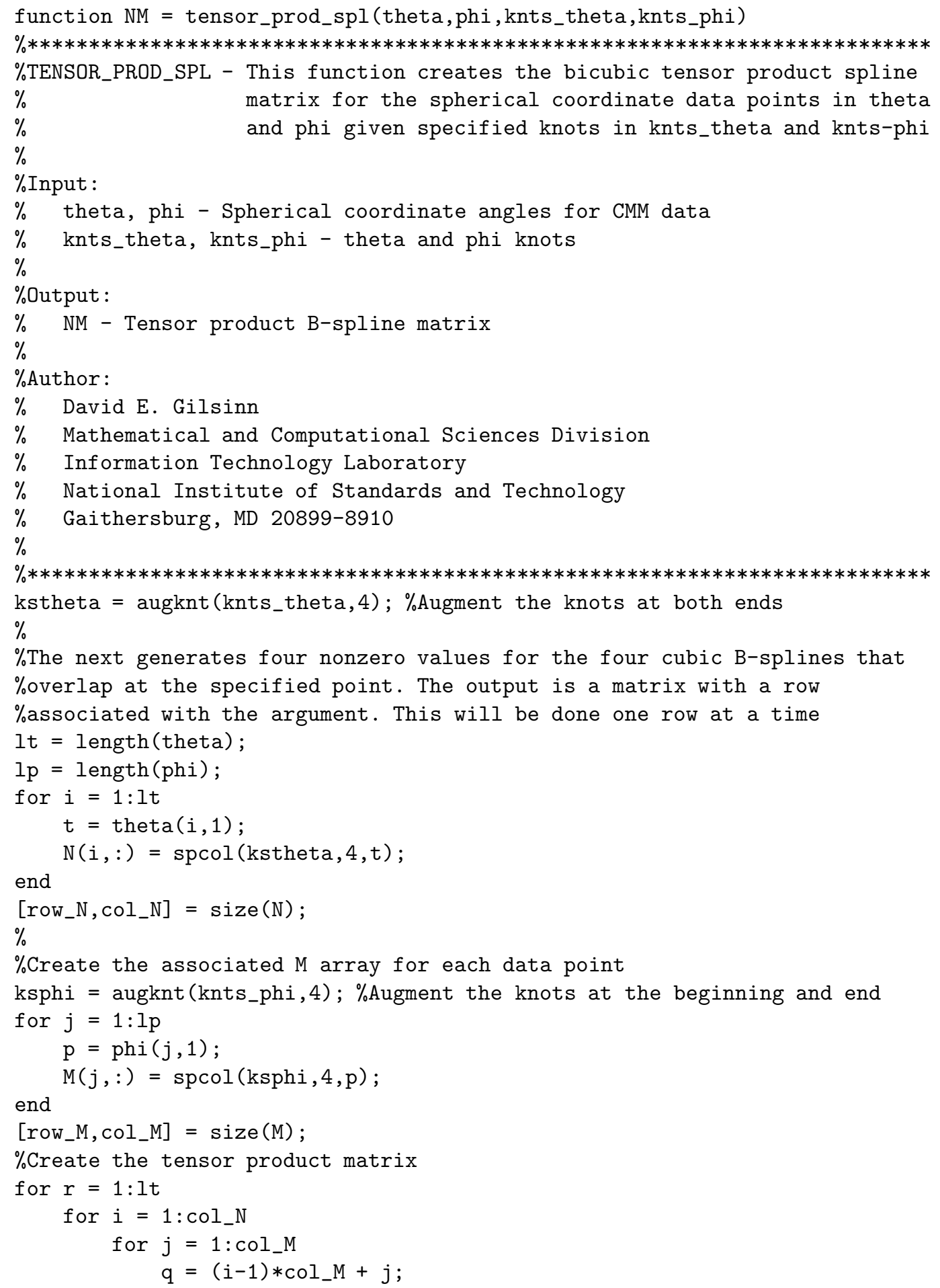




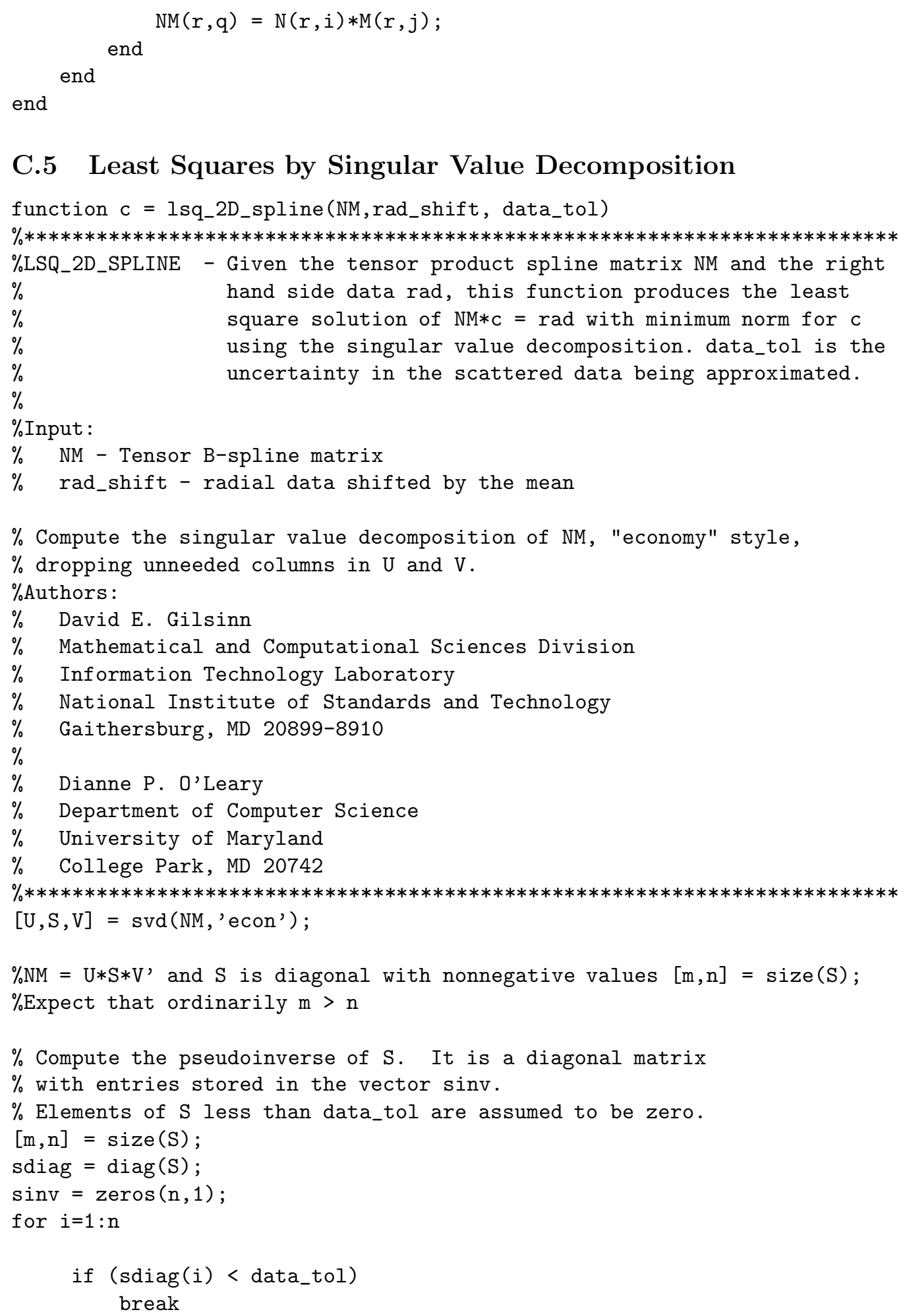

\section{C.5 Least Squares by Singular Value Decomposition}


end

$\operatorname{sinv}(i)=1 / \operatorname{sdiag}(i)$

end

$\%$ Form the solution vector as the pseudoinverse of NM times rad_shift.

$\mathrm{c}=\mathrm{V} *\left(\operatorname{sinv} \cdot *\left(\mathrm{U}^{\prime} * \mathrm{rad}_{-} \operatorname{shift} \mathrm{t}\right)\right)$

\section{C.6 Generating Data Points on a Surface Grid}

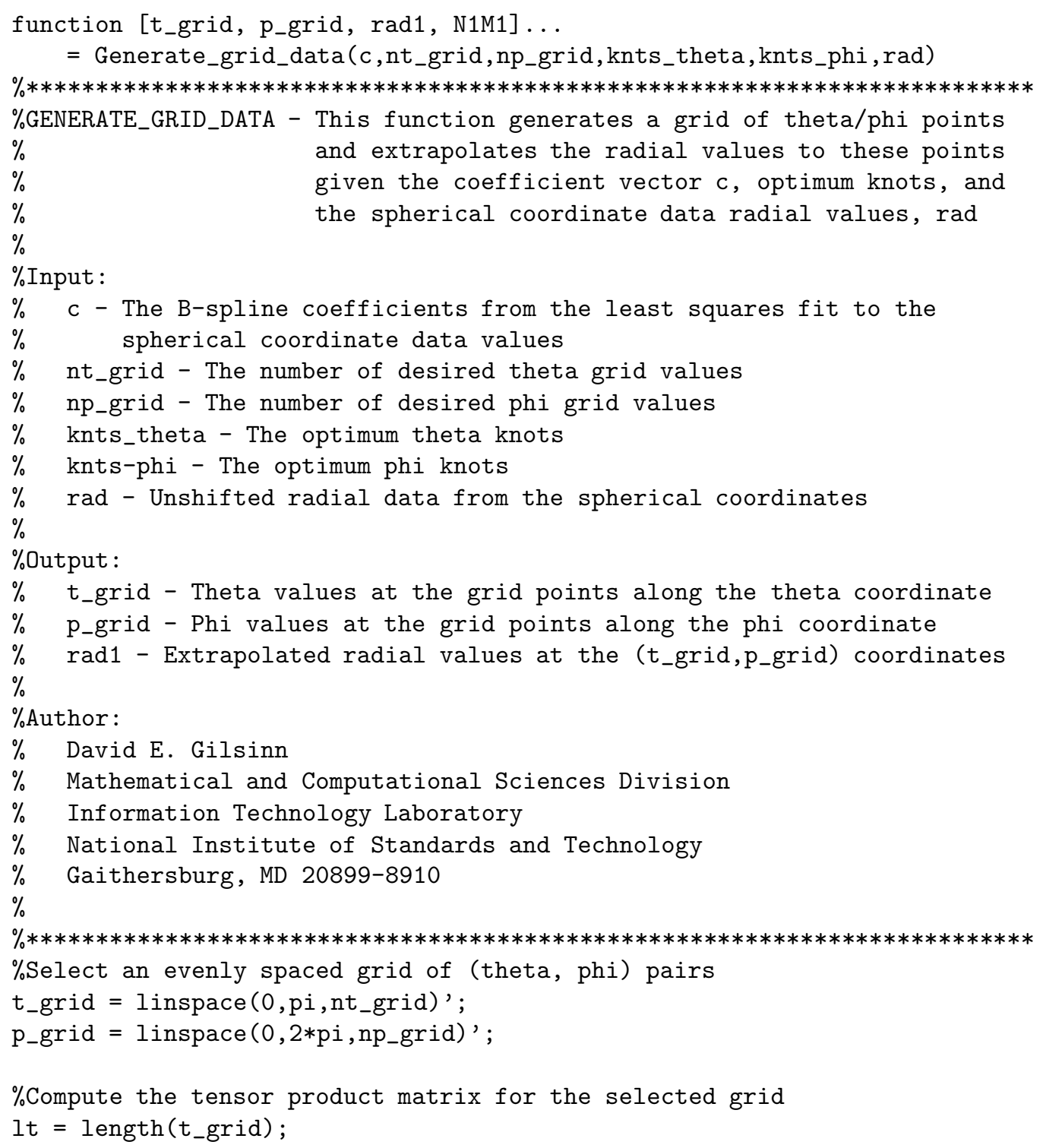




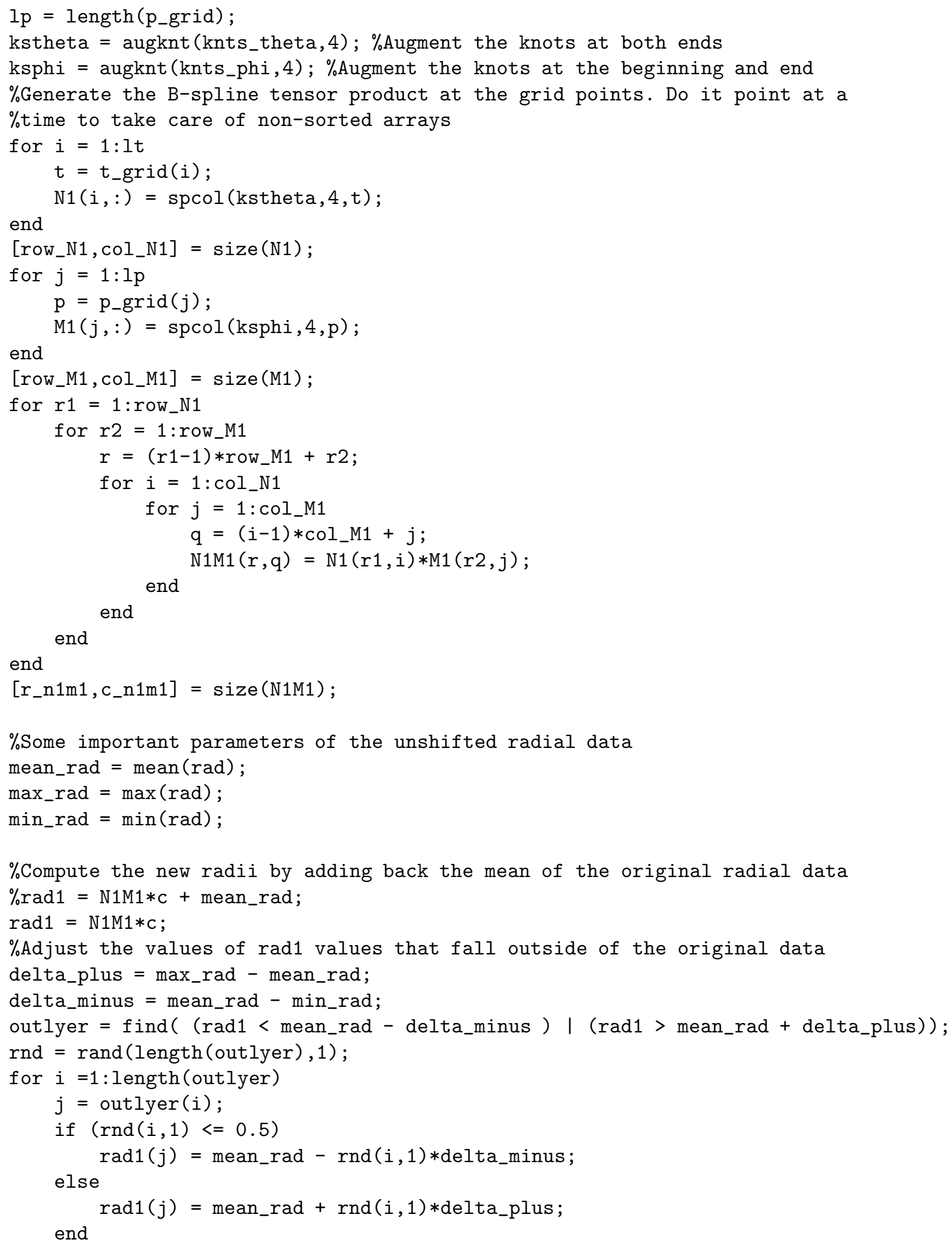




\section{C.7 Computing the Volume Based on Grid Values}

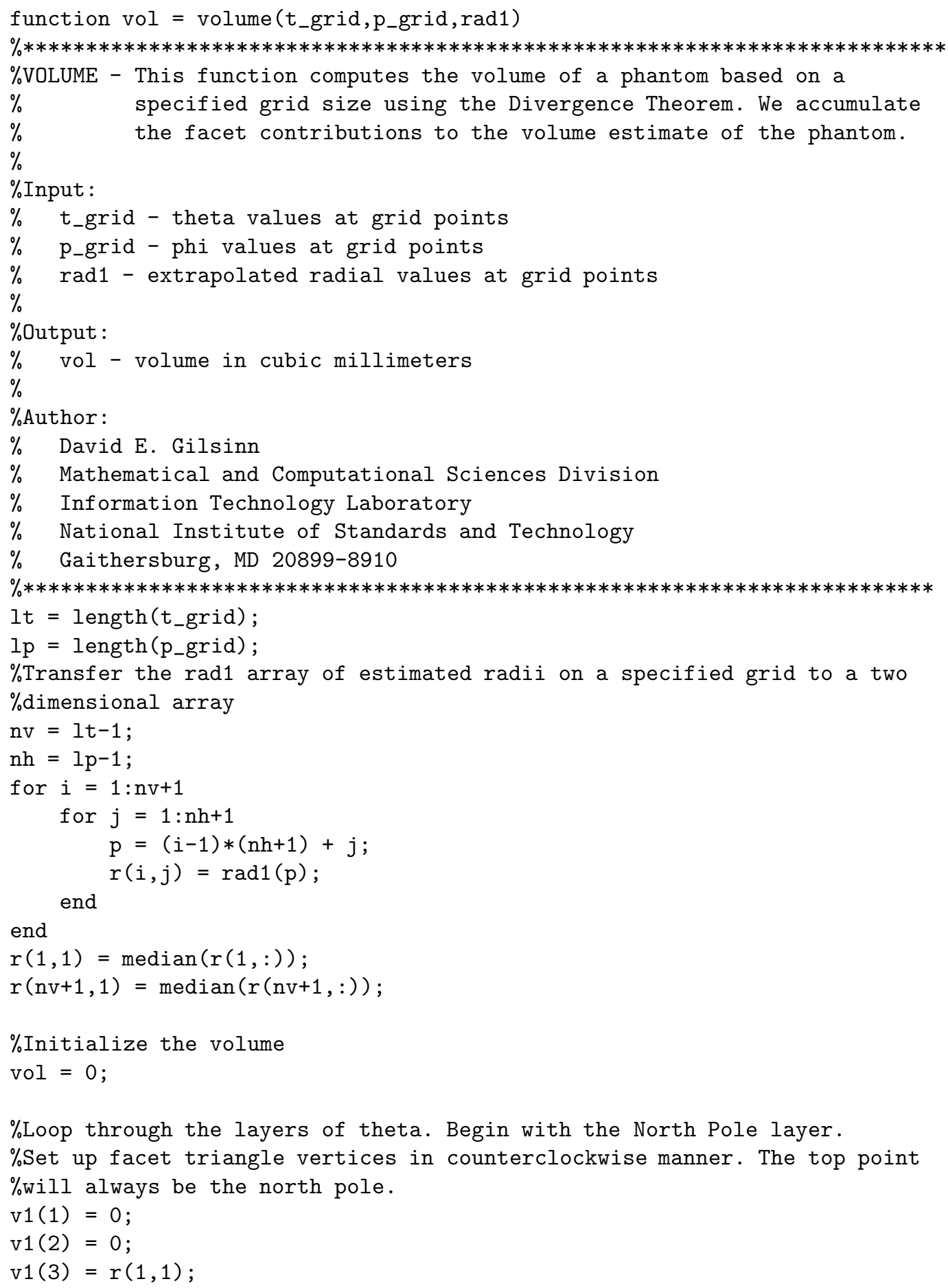




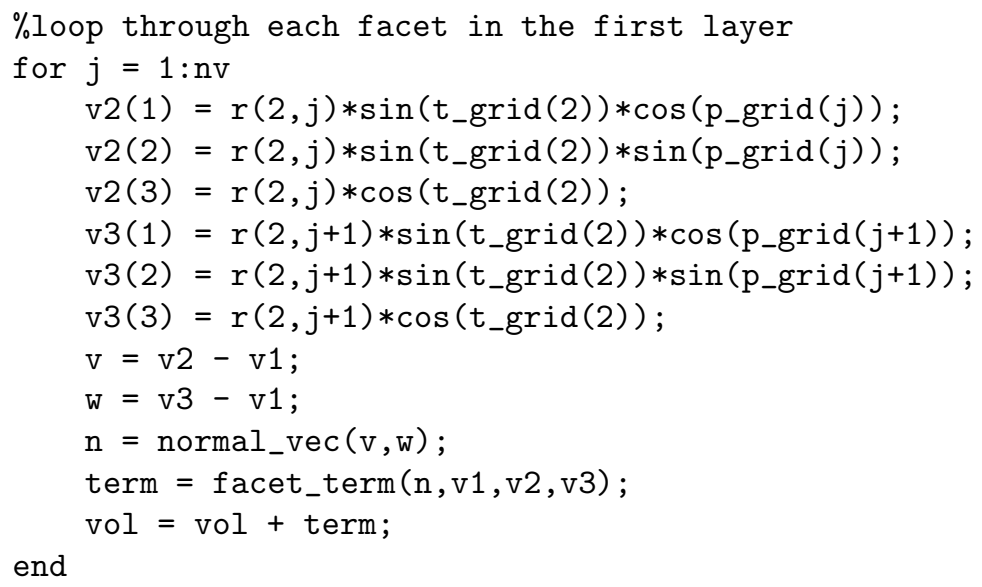

\%Next loop through layers 2 through $\mathrm{nv}-1$. There are $\mathrm{nh} 4$-vertex patches at $\%$ each level. Each patch will be divided into two triangles. The four $\%$ vertices of the patches will be identified in counterclockwise order as $\%\left(t \_g r i d(i), p \_g r i d(j), r(i, j)\right)$, 


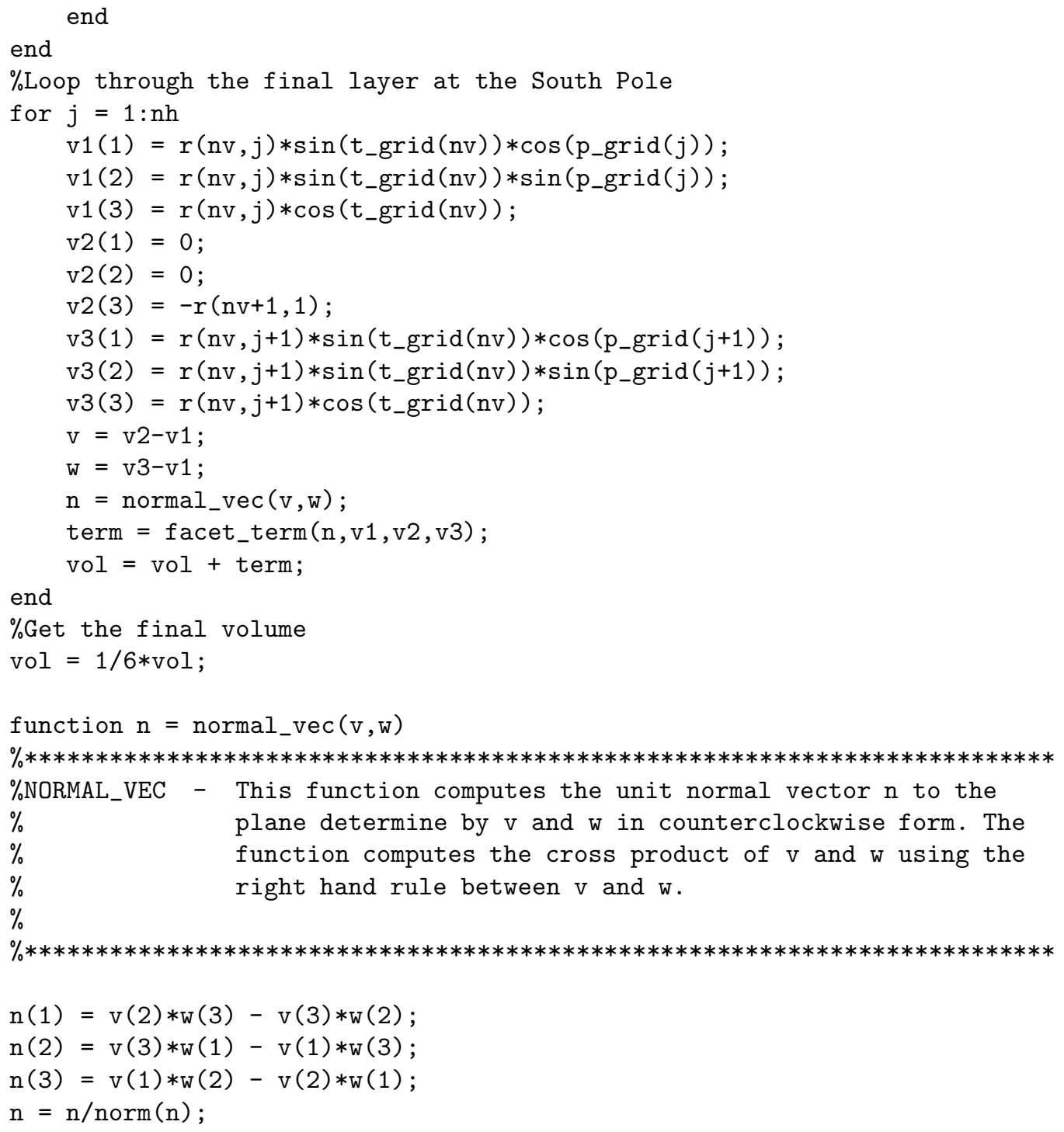

